University of Tennessee Health Science Center

UTHSC Digital Commons

\title{
Probing the Role of Nascent Helicity in Protein Function: p27kip1 as a Regulator of the Cell Cycle
}

Steve Otieno

University of Tennessee Health Science Center

Follow this and additional works at: https://dc.uthsc.edu/dissertations

Part of the Cells Commons

\section{Recommended Citation}

Otieno, Steve, "Probing the Role of Nascent Helicity in Protein Function: p27kip1 as a Regulator of the Cell Cycle" (2007). Theses and Dissertations (ETD). Paper 192. http://dx.doi.org/10.21007/ etd.cghs.2007.0231.

This Dissertation is brought to you for free and open access by the College of Graduate Health Sciences at UTHSC Digital Commons. It has been accepted for inclusion in Theses and Dissertations (ETD) by an authorized administrator of UTHSC Digital Commons. For more information, please contact jwelch30@uthsc.edu. 


\title{
Probing the Role of Nascent Helicity in Protein Function: p27kip1 as a Regulator of the Cell Cycle
}

\begin{abstract}
p $27^{\text {kip } 1}$ binds to and regulates the activity of cyclin-dependent kinases (Cdks) which are the master timekeepers of the cell division cycle. Members of the p27 family of proteins, also including p21 and p57, are called cyclin-dependent kinase inhibitors (CKIs). The amino terminal domain of p27 inhibits Cdk activity and is referred to as the kinase inhibitory domain (KID). The KID is comprised of a cyclin-binding domain (D1) and a Cdk binding domain (D2) joined by a 22 residue linker domain (LH). Structural analysis of the KID in solution before binding its Cdk targets revealed that D1 and D2 are largely unstructured and that the linker domain exhibits nascent helical characteristics. The nascent helical structure of the linker domain is conserved amongst the CKI proteins and I hypothesize that it is an important determinant of their functional properties. To test this hypothesis, I probed the interactions of peptides corresponding to D1 and D2 (LH domain deletion mutant) with the Cdk2/cyclin A complex. Results from isothermal titration calorimetry (ITC) and kinase inhibition assays, show that the interaction and of either D1 or D2 with the Cdk2/cyclin A complex is less favorable relative to that of the p27-KID and that the D2 peptide cannot completely inhibit Cdk2/cyclin A kinase activity. These results indicate that the LH domain that couples D1 and D2 is necessary for the function of p27 as a Cdk inhibitor.
\end{abstract}

Despite having a conserved structure, the linker domains of the Cip/Kip proteins do not have a conserved sequence. However, the sequence of this domain in each Cip/Kip protein in different species is well conserved suggesting that sequence may play a role in the function of these proteins. I explored this sequence-divergence/structure-conservation relationship of the linker domains of the Cip/Kip proteins by constructing chimeric p27-KID molecules in which the p27 linker domain was replaced with the corresponding segments of either p21 or p57. ITC and kinase inhibition assay results show that the chimeric molecules bind and inhibit Cdk2/cyclin A in a manner similar to that of p27-KID. Moreover, thermal denaturation studies show that the complexes formed by these proteins bound to Cdk2/cyclin A have comparable melting temperatures. Taken together, these results indicate that the different linker domains, despite their structural differences, play similar structural roles in p27 binding to Cdk2/cyclin A.

In a third study, I investigated the consequences of perturbing the conserved structure of the linker domain. I successfully designed and prepared p27 variants in which domain LH was either more or less helical with respect to the wild-type protein. The secondary structural properties of the mutants were characterized by circular dichroism spectropolarimetry (CD). Thermal denaturation experiments showed that the ternary complexes of the $\mathrm{p} 27$ variants bound to Cdk2/cyclin A were less stable compared to the ternary complex formed by wild- type $\mathrm{p} 27\left(\mathrm{p} 27-\mathrm{KID}^{\mathrm{wt}}\right)$. Thermodynamic analysis showed that there was a decrease in the enthalpy of association of the mutants with Cdk2/cyclin A relative to $\mathrm{p27-KID}{ }^{\mathrm{wt}}$. The free energies of binding varied within a much narrower range. In addition, in vitro Cdk2 inhibition assays showed that the 27 variants exhibited disparate inhibitory potencies. Further, when we over-expressed the 127 variants in mouse fibroblasts, they were less effective in causing cell cycle arrest relative to wildtype $\mathrm{p} 27$. These results indicate that the conserved structure of the linker domain is important for p27 function. It seems that nature has selected the sequence of the p27 LH domain to be partially structured and that bolstering or eliminating this structure is deleterious to function; intrinsic structure is critical for function.

\section{Document Type}

Dissertation 


\section{Degree Name}

Doctor of Philosophy (PhD)

\section{Program}

Molecular Sciences

\section{Research Advisor}

Richard Kriwacki, Ph.D.

\section{Keywords}

p27, cyclin-dependent kinase inhibitors, isothermal titration calorimetry, intrinsically unstructured proteins, cell cycle, thermal denaturation, nascent helix, entropic cost, cell cycle arrest, circular dichroism

\section{Subject Categories}

Cells | Medicine and Health Sciences 


\title{
PROBING THE ROLE OF NASCENT HELICITY IN PROTEIN FUNCTION: p27 ${ }^{\mathrm{kip} 1}$ AS A REGULATOR OF THE CELL CYCLE
}

\author{
A Dissertation \\ Presented for \\ The Graduate Studies Council \\ The University of Tennessee \\ Health Science Center
}

\author{
In Partial Fulfillment \\ Of the Requirements for the Degree \\ Doctor of Philosophy \\ From The University of Tennessee
}

By

Steve Biko Otieno

May 2007 
Copyright $@ 2007$ by Steve Biko Otieno

All rights reserved 


\section{Dedication}

This dissertation is dedicated to my father, the late Mr. Elly Otieno, and my mother, Mrs. Margaret Otieno for their unwavering support. 


\section{Acknowledgements}

I would like to acknowledge all those who supported and assisted me during my research. First, I would to thank Dr. Richard Kriwacki for his tutelage and for giving me an opportunity to work in his laboratory. My gratitude is also extended to all members of the Kriwacki laboratory, the Structural Biology Department at St. Jude Children's Research Hospital, and the Department of Molecular Sciences at The University of Tennessee Health Science Center. I would also like to thank my family for the support they have accorded me in the course of graduate school. Finally, I acknowledge the National Institutes of Health $(\mathrm{NIH})$ and the American Lebanese and Syrian Associated Charities (ALSAC) for funding my research project. 


\begin{abstract}
p $27^{\text {kip } 1}$ binds to and regulates the activity of cyclin-dependent kinases (Cdks) which are the master timekeepers of the cell division cycle. Members of the p27 family of proteins, also including p21 and p57, are called cyclindependent kinase inhibitors (CKls). The amino terminal domain of p27 inhibits Cdk activity and is referred to as the kinase inhibitory domain (KID). The KID is comprised of a cyclin-binding domain (D1) and a Cdk binding domain (D2) joined by a 22 residue linker domain (LH). Structural analysis of the KID in solution before binding its Cdk targets revealed that D1 and D2 are largely unstructured and that the linker domain exhibits nascent helical characteristics. The nascent helical structure of the linker domain is conserved amongst the CKI proteins and I hypothesize that it is an important determinant of their functional properties. To test this hypothesis, I probed the interactions of peptides corresponding to D1 and D2 (LH domain deletion mutant) with the Cdk2/cyclin A complex. Results from isothermal titration calorimetry (ITC) and kinase inhibition assays, show that the interaction and of either D1 or D2 with the Cdk2/cyclin A complex is less favorable relative to that of the p27-KID and that the D2 peptide cannot completely inhibit Cdk2/cyclin A kinase activity. These results indicate that the LH domain that couples D1 and D2 is necessary for the function of p27 as a Cdk inhibitor.
\end{abstract}

Despite having a conserved structure, the linker domains of the Cip/Kip proteins do not have a conserved sequence. However, the sequence of this 
domain in each Cip/Kip protein in different species is well conserved suggesting that sequence may play a role in the function of these proteins. I explored this sequence-divergence/structure-conservation relationship of the linker domains of the Cip/Kip proteins by constructing chimeric p27-KID molecules in which the p27 linker domain was replaced with the corresponding segments of either p21 or p57. ITC and kinase inhibition assay results show that the chimeric molecules bind and inhibit Cdk2/cyclin A in a manner similar to that of p27-KID. Moreover, thermal denaturation studies show that the complexes formed by these proteins bound to Cdk2/cyclin A have comparable melting temperatures. Taken together, these results indicate that the different linker domains, despite their structural differences, play similar structural roles in p27 binding to Cdk2/cyclin A.

In a third study, I investigated the consequences of perturbing the conserved structure of the linker domain. I successfully designed and prepared p27 variants in which domain LH was either more or less helical with respect to the wild-type protein. The secondary structural properties of the mutants were characterized by circular dichroism spectropolarimetry (CD). Thermal denaturation experiments showed that the ternary complexes of the p27 variants bound to Cdk2/cyclin A were less stable compared to the ternary complex formed by wild-type $\mathrm{p} 27\left(\mathrm{p} 27-\mathrm{KID}^{\mathrm{wt}}\right)$. Thermodynamic analysis showed that there was a decrease in the enthalpy of association of the mutants with Cdk2/cyclin A relative to $\mathrm{p} 27-\mathrm{KID}^{\mathrm{wt}}$. The free energies of binding varied within a much narrower range. In addition, in vitro Cdk2 inhibition assays showed that the p27 variants exhibited disparate inhibitory potencies. Further, when we over-expressed the p27 
variants in mouse fibroblasts, they were less effective in causing cell cycle arrest relative to wild-type $\mathrm{p} 27$. These results indicate that the conserved structure of the linker domain is important for p27 function. It seems that nature has selected the sequence of the p27 LH domain to be partially structured and that bolstering or eliminating this structure is deleterious to function; intrinsic structure is critical for function. 


\section{Table of Contents}

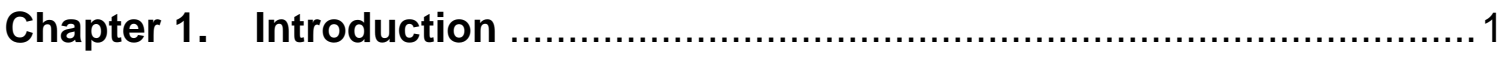

1.1. Cyclin-dependent kinases ................................................... 3

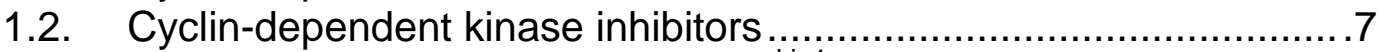

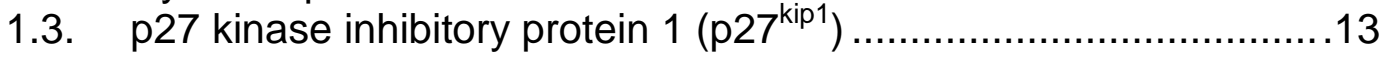

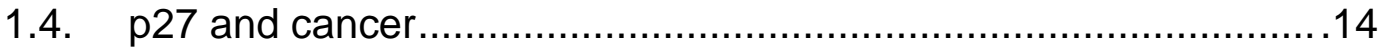

1.5. $\mathrm{p} 27$ is an intrinsically unstructured protein................................17

1.6. Thermodynamics of the association of p27 and Cdk2/cyclin A.....21

1.7. Project hypotheses and strategy .......................................24

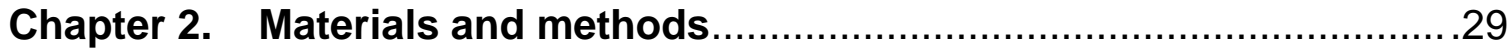

2.1. Design of the more and less helical p27-KID linker domain mutants

2.2. Preparation of mutagenesis cassettes.....................................34

2.3. Preparation of cassettes for cloning Cip/Kip linker

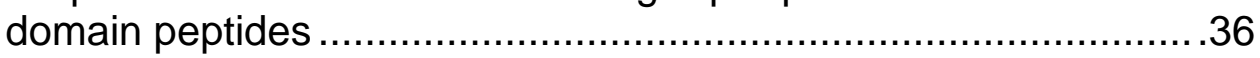

2.4. Preparation of Escherichia coli expression plasmids ....................38

2.5. Preparation of retroviral shuttle vectors .....................................44

2.6. Expression and purification of Cip/Kip linker domain

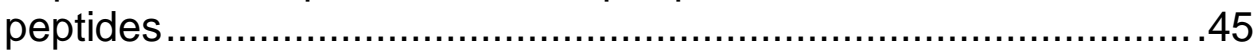

2.7. Expression and purification of p27-KID variants ..........................47

2.8. Expression and purification of cyclin $A$ and

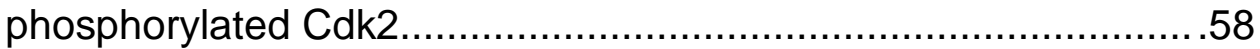

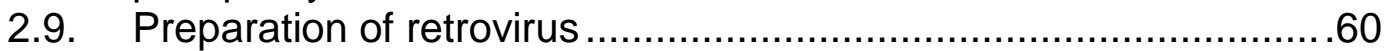

2.10. Viral transduction and cell cycle arrest analysis ..........................63

2.11. Protein concentration determination ..........................................64

2.12. Secondary structure analysis by circular

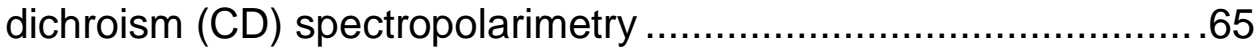

2.13. Thermal denaturation experiments .............................................66

2.14. Determination of thermodynamic parameters by ITC ..................67

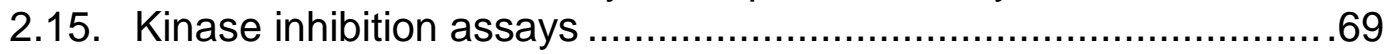

Chapter 3. The linker domains of proteins in the Cip/Kip family play similar structural roles

3.1. The nascent helical character of the linker domain 
is preserved in the chimeric mutants and the

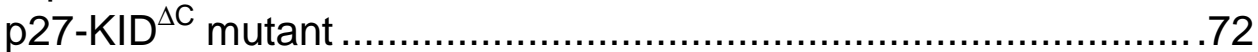

3.2. Binding thermodynamics determined by ITC .............................75

3.3. The p27-KID ${ }^{\mathrm{wt}}$ ternary complex is more stable than the corresponding complexes of its variants.

3.4. Relative inhibition potency determined by in vitro

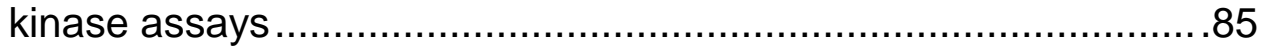

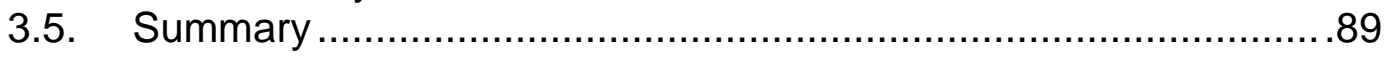

\section{Chapter 4. Consequences of perturbation of the structure} of the linker domain of p27.

4.1. Secondary structure analysis of the more and less helical p27-KID mutants

4.2. The free energy of the interaction of $\mathrm{p} 27-\mathrm{KID}$ with $\mathrm{P}-\mathrm{Cdk2/cyclin} \mathrm{A}$ does not decrease when the helical content in the linker domain of p27 is increased.

4.3. The ternary complexes formed by the linker helix mutants on binding P-Cdk2/cyclin A are thermodynamically less stable relative to that of $\mathrm{p} 27-\mathrm{KID}^{\mathrm{wt}}$

4.4. The linker helix mutants are less potent inhibitors of $\mathrm{P}-\mathrm{Cdk2/cyclin} \mathrm{A}$ kinase activity relative to $\mathrm{p} 27-\mathrm{KID}^{\mathrm{wt}}$

4.5. Perturbation of the structure of the linker domain is deleterious to p27 function in cells

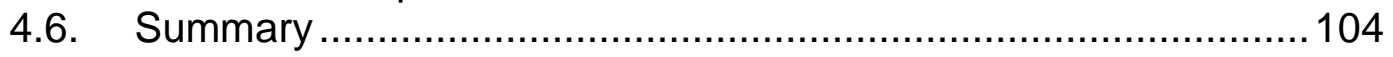

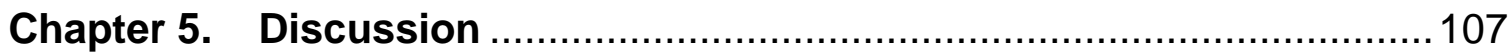

5.1. The linker domain of $\mathrm{p} 27$ is necessary for function.....................107

5.2. The linker domains of the Cip/Kip proteins play similar structural roles despite their sequence differences.

5.3. The role of the conserved residues in the linker domain of Cip/Kip proteins

5.4. Biophysical and functional effects of increasing, decreasing, or eliminating the helical structure of the linker domain of $p 27$

List of references 
A.1. Buffers ........................................................................ 140

A.2. Growth media .......................................................... 143

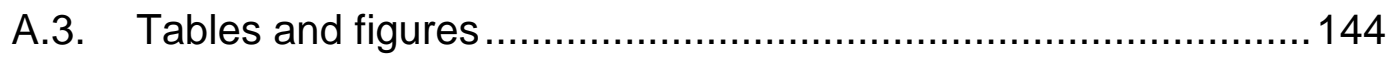

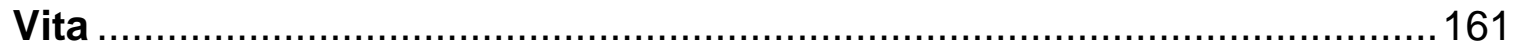




\section{List of Tables}

Table 1.1 Cyclins, cyclin-dependent kinases and their biological roles ...........5

Table 1.2 Alteration of the p27 protein in human malignancies ................... 16

Table 1.3 Biological functions of IUP's .................................................19

Table 2.1 Solvent exposure of the residues in the linker domain of p27 …...30

Table 2.2 Linker domain sequences for the p27-KID variants designed

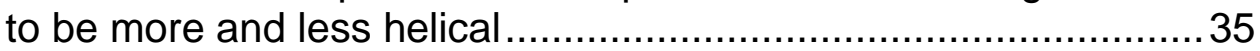

Table 3.1 Thermodynamic parameters determined by ITC at $25{ }^{\circ} \mathrm{C} \ldots \ldots \ldots \ldots . . . .77$

Table 3.2 Melting temperatures of ternary complexes formed by either p27-KID ${ }^{\text {wt }}$ or its variants bound to P-Cdk2/cyclin A complex...........84

Table 3.3 Inhibition potencies of the p27-KID variants ..............................87

Table 4.1 Thermodynamic binding parameters of the p27-KID mutants .......95

Table 4.2 Melting temperatures of the ternary complexes formed by the p27-KID mutants on binding the P-Cdk2/cyclin A complex......98

Table 4.3 Inhibition potencies of the p27-KID mutants .............................102

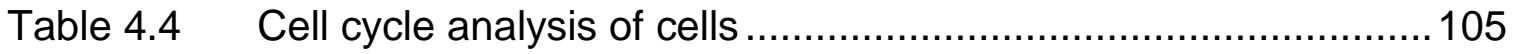

Table A.1 Sequences of oligonucleotides used in preparing mutagenesis cassettes for the p27-KID mutants

Table A.2 Reagents for overlap extension PCR …................................ 148

Table A.3 Thermocycler settings for the oePCR ......................................149

Table A.4 Sequences of oligonucleotides and primers used in preparing cloning cassettes for the Cip/Kip linker domain peptides............. 150

Table A.5 Cloning of the p27-KID linker domain mutants ......................... 151

Table A.6 Expression and solubility of the p27-KID linker domain mutants 


\section{List of Figures}

Figure 1.1 Schematic diagram of the eukaryotic cell division cycle .................2

Figure 1.2 Structures of the Cdk2/cyclin A (PDB; 1FIN), p19/Cdk6 (PDB; 1B18), and p16/Cdk6 (PDB; 1B17) ................................ 8

Figure 1.3 The Cip/Kip proteins regulate the activity of a broad range of Cdk/cyclin complexes

Figure 1.4 The structure of the p27/Cdk2/cyclin A complex (PDB; 1JSU) ......12

Figure 1.5 Sequence alignment of the KID domains of the Cip/Kip proteins.

Figure 1.6 Alignment of p27-LH domains from different species .................26

Figure 2.1 The structure of p27/Cdk2/cyclin A (PDB; 1JSU) showing the residues determined to be suitable for mutagenesis

Figure 2.2 Agarose gel analysis of the oePCR for the p27-KID ${ }^{-H}$ mutant .......37

Figure 2.3 The muticloning site of the pET28 vector ...................................39

Figure 2.4 The pET28-GST-His p27-LH vector construct...........................41

Figure 2.5 Schematic diagram of the cassette mutagenesis design ...............43

Figure 2.6 Purification of the p27 linker domain peptide (p27-LH) ................48

Figure 2.7 Mass spectrometry analyses of p27 and p21 linker domain

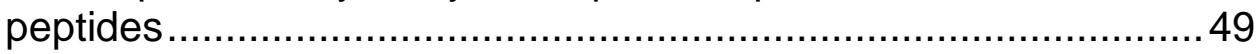

Figure 2.8 Mass spectrometry analysis of p57 linker domain peptide ...........50

Figure 2.9 Purification of the $\mathrm{p} 27-\mathrm{KID}^{-\mathrm{H}}$ mutant .......................................52

Figure 2.10 Mass spectrometry analyses of $\mathrm{p} 27-\mathrm{KID}^{\mathrm{wt}}$ and $\mathrm{p} 27-\mathrm{KID}^{\mathrm{p} 21 \text { helix }} \ldots \ldots . .53$

Figure 2.11 Mass spectrometry analyses of $\mathrm{p} 27-\mathrm{KID}^{\mathrm{p} 57 \mathrm{~h} e l i x}$ and $\mathrm{p} 27-\mathrm{KID}^{\Delta \mathrm{C}} \ldots . .54$

Figure 2.12 Mass spectrometry analyses of purified p27-KID ${ }^{+H}, \mathrm{p} 27-\mathrm{KID}^{-\mathrm{H}}$, and $\mathrm{p} 27-\mathrm{KID}^{\mathrm{loop}}$. 
Figure 2.13 SDS-PAGE analysis of purified p27-KID mutants ......................56

Figure 2.14 Mass spectrometry analysis of the D2 peptide ..........................57

Figure 3.1 CD spectra of the p21, p27, and p57 linker domain peptides ........73

Figure 3.2 CD spectra of p27-KID ${ }^{\mathrm{wt}}, \mathrm{p} 27-\mathrm{KID}^{\mathrm{p} 21 \mathrm{helix}}, \mathrm{p} 27-\mathrm{KID}^{\mathrm{p} 57 \mathrm{helix}}$, and

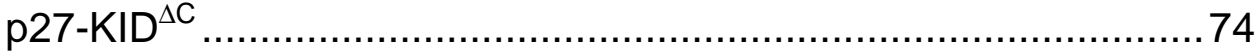

Figure 3.3 ITC binding isotherms for the p27-KID variants ..........................78

Figure 3.4 Thermal denaturation curves of the p27-KID mutants ..................83

Figure 3.5 Inhibition of P-Cdk2/cyclin A kinase activity by the D2 peptide......86

Figure 3.6 Inhibition of P-Cdk2/cyclin A kinase activity by the

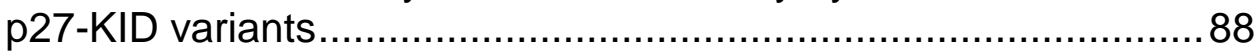

Figure 4.1 CD spectra of the p27-KID linker domain mutants ..................... 92

Figure 4.2 ITC binding isotherms for the interaction of the p27-KID mutants with the P-Cdk2/cyclin A complex..................................94

Figure 4.3 Thermal denaturation curves for the p27-KID variants ................97

Figure 4.4 P-Cdk2/cyclin A kinase inhibition by the p27-KID mutants ......... 101

Figure A.1 A schematic representation of oePCR ....................................153

Figure A.2 Agarose gel analysis of the oePCR for the p27 linker domain

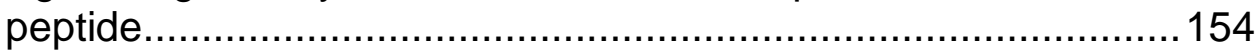

Figure A.3 Sequencing results for the pET28p27-KID plasmid ....................155

Figure A.4 Sequencing results for the pET28flp27 plasmid ....................... 156

Figure A.5 Vector map for the MSCV-I-GFP plasmid................................. 157

Figure A.6 Sequences of the MSCV-I-GFP plasmids ............................. 158

Figure A.7 Flow cytometry analysis of GFP positive cells ......................... 159

Figure A.8 Cell cycle arrest analysis of the p27-KID mutants ....................160 


\section{List of Abbreviations}

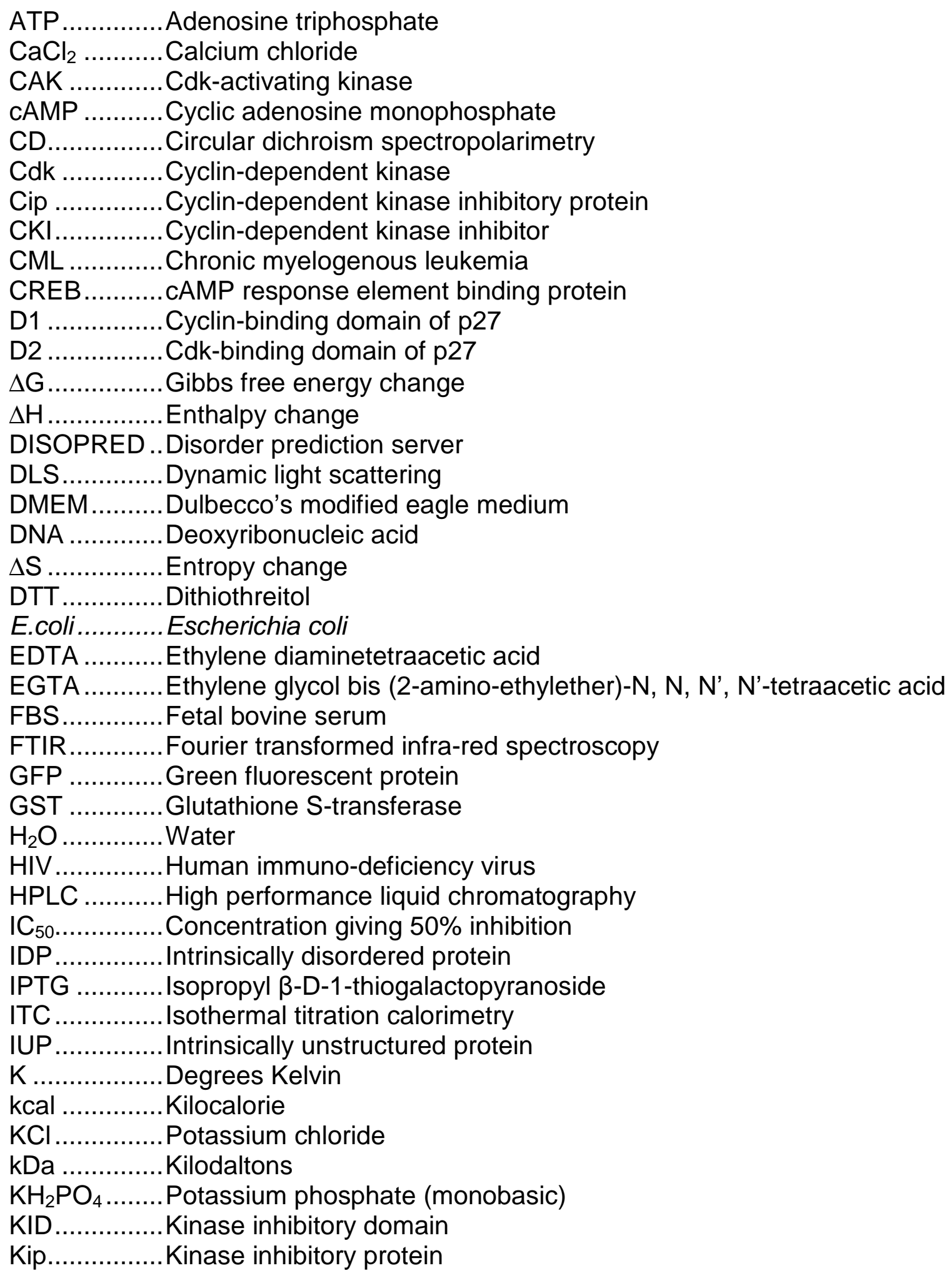




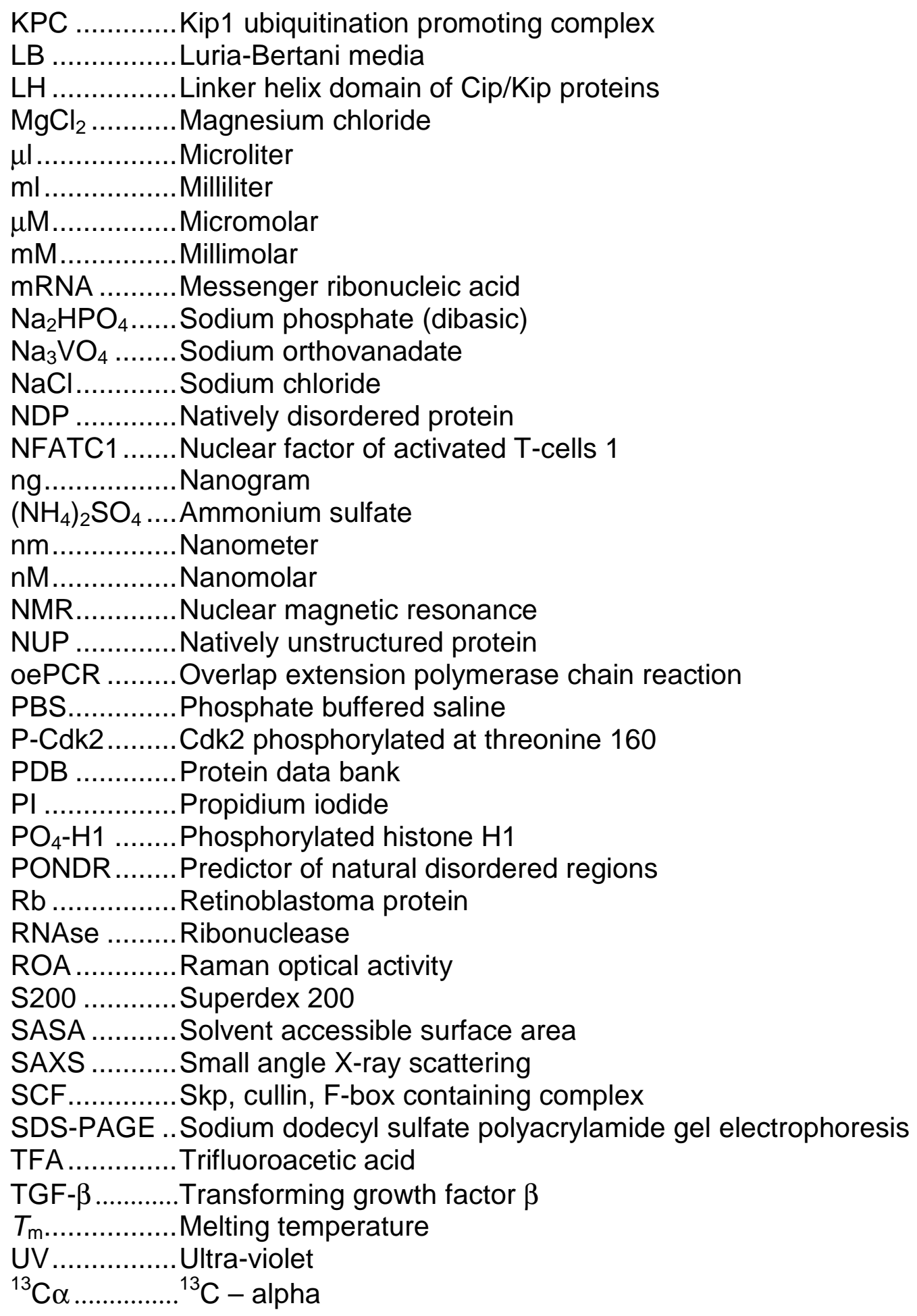




\section{Chapter 1: Introduction}

Self-replication is a fundamental property of eukaryotic cells. A complex, coordinated succession of events ensures that the parent cell is divided into two identical daughter cells. The sequence of events that take place between one cell division and the next is referred to as the cell division cycle or the cell

cycle. ${ }^{1,2}$ This series of events can be separated into four distinct phases:

- G1 phase - characterized by cell growth, synthesis of new organelles, increased protein synthesis, and an accelerated metabolic rate.

- $\quad S$ phase - characterized by synthesis of deoxyribonucleic acid (DNA).

- $\quad$ G2 phase - the cells prepare for mitosis and checkpoints ensure that any insults to the genome since the previous division are not propagated. The G1, S and G2 phases are collectively referred to as interphase.

- $\quad \mathrm{M}$ phase - the genetic material is divided into two identical fractions (mitosis) and the cell divides into two daughter cells each containing one half of the genetic material (cytokinesis).

Proliferating cells may exit the cell cycle temporarily, or reversibly, and enter into a state called quiescence or $G_{0}$ phase. Cells typically enter the $G_{0}$ phase from the G1 phase. Figure 1.1 shows the various phases of the cell cycle. The cell cycle is one of the most extensively regulated processes in eukaryotic organisms. A number of proteins including kinases, kinase activators and inhibitors, phosphatases, and transcription factors, just to mention but a few, are involved in the modulation of the cell division cycle. 


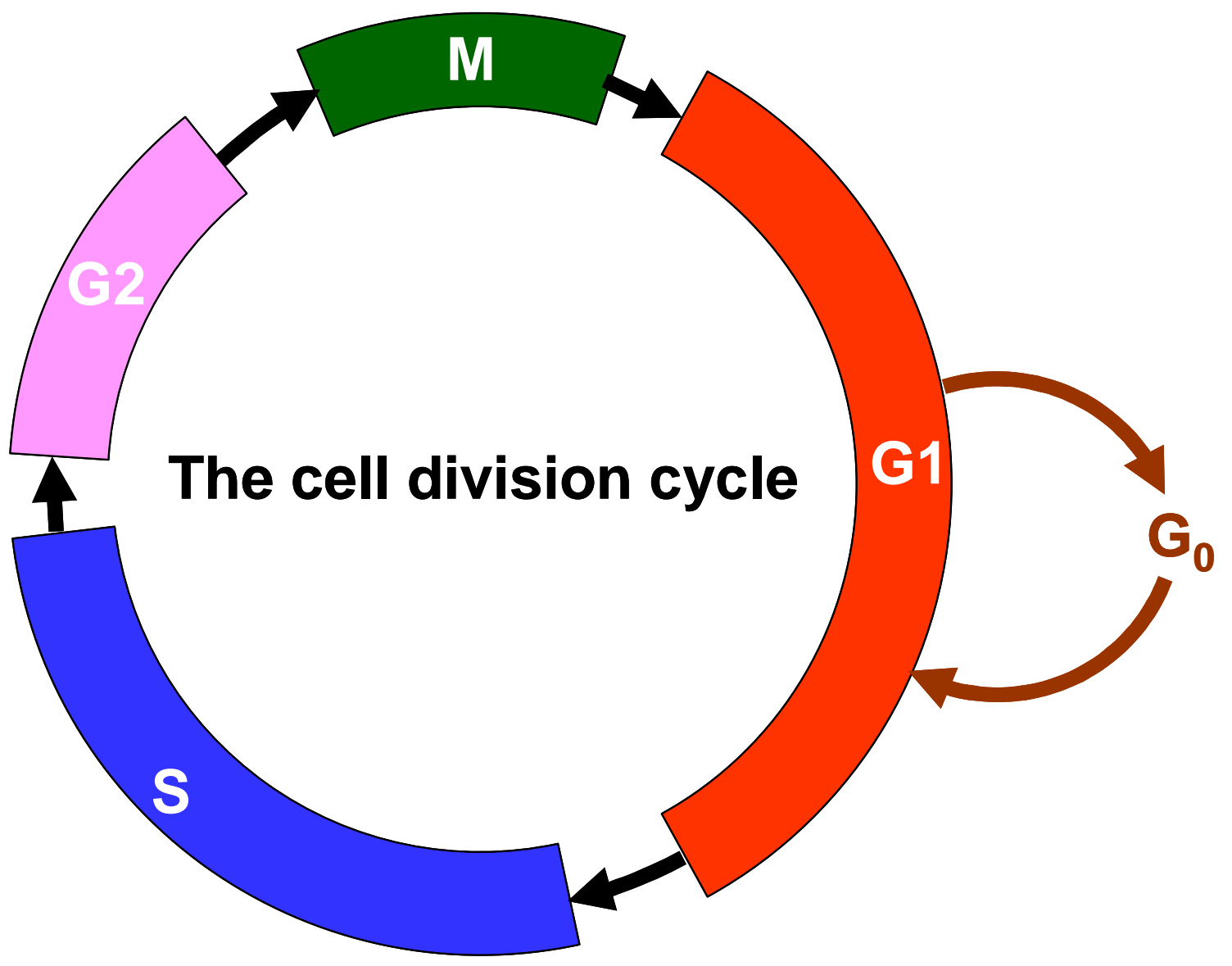

Figure 1.1 Schematic diagram of the eukaryotic cell division cycle. 


\subsection{Cyclin-dependent kinases}

Strict temporal control of cell cycle progression is required for faithful duplication of eukaryotic cells. This is achieved predominantly through the activity of a family of highly conserved serine/threonine kinases called cyclindependent kinases (Cdk's). ${ }^{3-11}$ The Cdk's govern phase transitions during the cell cycle in a timely manner and are referred to as "master timekeepers" of the cell cycle. ${ }^{12}$ Two events are required for complete activation of the Cdk's: (i) a regulatory subunit called a cyclin binds to the Cdk; ${ }^{13-19}$ and (ii) the Cdk is phophorylated on a conserved threonine residue (T160 in Cdk2) by a family of proteins called Cdk-activating kinases (CAK's) ${ }^{20-27}$ The kinase active site is located in the Cdk subunit of the heterodimer. Upon activation, the Cdk's phosphorylate a number of substrates orchestrating an orderly progression of the cell cycle from one phase to the next. As an example, Cdk4/cyclin D, Cdk2/cyclin A and Cdk2/cyclin E phosphorylate the retinoblastoma $(\mathrm{Rb})$ protein facilitating the transition of proliferating cells from the $\mathrm{G} 1$ to the S phase.$^{28-31}$ In the hypophosphorylated state, the $\mathrm{Rb}$ protein represses transcription by binding and negatively regulating the activity of transcription factors such as the E2F family of transcription factors, chromatin remodeling complexes, and histone deacetylases. ${ }^{32-34}$ Phosphorylation of the Rb protein by the Cdk complexes relieves its repressor activity and activates the transcription factors culminating in progression from $\mathrm{G} 1$ to $\mathrm{S}$ phase.

The protein level of the cyclins oscillates depending on the phase of the cell cycle ensuring that the Cdk's are activated at the appropriate time. ${ }^{35}$ The 
abundance of the cyclins in any cell cycle phase is regulated through programmed synthesis and degradation. ${ }^{17}$ A classic example is the regulation of protein levels of cyclin E and cyclin B. The synthesis of cyclin E is induced in late G1 and its level peaks at the $\mathrm{G} 1$ to $\mathrm{S}$ transition. ${ }^{36}$ As previously mentioned, cyclin $\mathrm{E}$ activates Cdk2 and promotes $\mathrm{G} 1$ to $\mathrm{S}$ transition. However, as the $\mathrm{S}$ phase starts and progresses, cyclin E activity is no longer required and its protein levels are reduced by degradation. ${ }^{17} \mathrm{Cdk} 1 / \mathrm{cyclin} B$ is required for the G2/M transition and cyclin B synthesis is induced in late S phase. ${ }^{37}$ The cyclin B accumulates in complexes with Cdk1 as the cells approach the $\mathrm{G} 2$ to $\mathrm{M}$ transition. ${ }^{37}$ When the cells enter the $M$ phase, cyclin $B$ is rapidly degraded. ${ }^{37}$ In addition to cyclins, other regulators of cell cycle are also temporally degraded to ensure proper progression of the cell cycle ${ }^{35,38}$ Protein degradation is therefore an important regulatory mechanism in cell cycle progression.

The Cdk's were originally discovered in yeast where they were shown to be indispensable players in progression of the cell cycle. The Saccharomyces cerevisiae Cdk, Cdc2, and the Saccharomyces pombe Cdk, Cdc28, were the first to be identified. ${ }^{39-41}$ The human orthologs were soon discovered based on their capacity to complement yeast mutants. ${ }^{42}$ In yeast, only a single Cdk regulates cell cycle events. In contrast, the cell cycle in higher eukaryotes is regulated by multiple Cdk's. To date, 11 Cdk's (Cdk 1-11) and 9 Cdk-related proteins have been identified. ${ }^{4}$ It is important to note that not all the Cdk's and Cdk-related proteins are involved in cell cycle progression (Table 1.1). Only Cdk's 1, 2, 4, and 6 have been directly implicated in cell cycle progression. On the other hand, 
Table 1.1 Cyclins, cyclin-dependent kinases and their biological roles.

\begin{tabular}{|c|c|c|}
\hline Cdk & Associated cyclin(s) & Function \\
\hline Cdk1 & Cyclins A, B, E & Regulation of G1-S, G2-M transitions \\
\hline Cdk2 & Cyclins A, E & Regulation of G1-S transition \\
\hline Cdk3 & Cyclins E, A, C & Cell cycle exit \\
\hline Cdk4 & Cyclins D1, D2, D3 & Regulation of G1-S transition \\
\hline Cdk5 & Cyclins p35, p39 & Neuronal regulation \\
\hline Cdk6 & Cyclins D1, D2, D3 & Regulation of G1-S transition \\
\hline Cdk7 & Cyclin $\mathrm{H}$ & Cdk-activating kinase \\
\hline Cdk8 & Cyclin C & Regulation of transcription \\
\hline Cdk9 & Cyclins T1, T2, K & Regulation of transcription \\
\hline Cdk10 & Unknown & Regulation of transcription \\
\hline Cdk11 & Cyclins L1, L2 & Regulation of transcription \\
\hline
\end{tabular}


Cdk7 acts as a CAK regulating the activity of other Cdk's ${ }^{43,44}$ and Cdk5 is predominantly expressed in neurons where it is involved in the regulation of neuronal functions including synaptic transmission and cytoskeletal remodeling. ${ }^{45,46}$ Other Cdks like Cdk8 and Cdk9 are mainly involved in regulation of transcription. ${ }^{47-50}$ The best characterized members of the Cdk family are those involved in cell cycle progression.

Although Cdk's have different regulatory roles in cells, their catalytic domains are highly conserved and have a similar three-dimensional structure. Past studies have shown that in the unbound Cdk's, the active site is not properly configured for catalysis. ${ }^{51}$ The binding of a cyclin subunit and CAK phosphorylation induces conformational changes which enable the Cdk active site to become catalytically competent. ${ }^{52,53}$ In addition to Cdk activation, the cyclin subunit also contains the binding site for a number Cdk substrates. ${ }^{54}$ The binding site is composed of a conserved set of residues that form a hydrophobic patch. Key cell cycle regulatory proteins including $\mathrm{Rb}$ have been shown to bind to this substrate-binding motif. ${ }^{54}$ Cdk activity is also regulated by inhibition.

Phosphorylation within the N-terminal region of Cdk's can lead to inactivation. This inactivating modification occurs on a single tyrosine residue in Cdk's 4 and 6 (Y17 and Y24 of Cdk4 and Cdk6, respectively), while Cdk1 and Cdk2 are phosphorylated on adjacent threonine and tyrosine residues (T14 and Y15 on Cdk1 and Cdk2). ${ }^{55-59}$ Previous studies have demonstrated that Cdk1 and Cdk2 are phosphorylated by Wee1 and Myt1 kinases. ${ }^{60-64}$ However, the kinase(s) that phosphorylate Cdk's 4 and 6 have not yet been identified. This 
inactivating phosphorylation event is reversed by the activity of Cdc25 family of phosphatases. ${ }^{65-67}$ In addition to phosphorylation, Cdk activity is also inhibited by a family of proteins termed the cyclin-dependent kinase inhibitors.

\subsection{Cyclin-dependent kinase inhibitors}

The cyclin-dependent kinase inhibitors (CKl's) negatively regulate the activity of the Cdk's. ${ }^{68,69}$ In humans, CKI's are divided into two families: the Ink4 family ${ }^{70-73}$ that includes $\mathrm{p} 15^{\operatorname{lnk} 4 \mathrm{~b}}, \mathrm{p} 16^{\operatorname{lnk} 4 a}, \mathrm{p} 18^{\operatorname{lnk} 4 \mathrm{c}}$, and $\mathrm{p} 19^{\ln k 4 \mathrm{~d}}$; and the Cip/Kip family ${ }^{74-88}$ that includes $p 21^{\text {Waf1/Cip1/Sdi1/Cap20 }}$, p2 $7^{\text {Kip1 }}$, and p57 ${ }^{\text {Kip2 }}$. The Ink4 family inhibits Cdk activity by binding to the catalytic subunit of Cdk's 4 and 6 abolishing their ability to form active complexes with D-type cyclins ${ }^{89}$ (cyclin D1, cyclin D2 and cyclin D3; expressed in an organ and tissue specific manner ${ }^{37}$ ). Figure 1.2 shows the structures of $p 19 / \mathrm{Cdk6}, \mathrm{p} 16 / \mathrm{Cdk} 6$ and Cdk2/cyclin A complexes. The PDB accession numbers for the p16/Cdk6, p19/Cdk6 and Cdk2/cyclin A are $1 \mathrm{BI}, 1 \mathrm{BI}$, and $1 \mathrm{FIN}$, respectively. The binding of $\mathrm{p} 19$ and $\mathrm{p} 16$ to Cdk6 distorts the cyclin binding site (compare with the cyclin binding site in the Cdk2/cyclin A complex). By blocking cyclin D from binding, p19 and p16 maintain Cdk6 in an inactive state. Cdks adopt the bilobed fold common to many protein kinases. ${ }^{90}$ This fold is comprised of an $\mathrm{N}$-terminal lobe that is largely comprised of $\beta$-sheets and a C-terminal lobe that is mainly comprised of $\alpha$-helices. The binding of $\mathrm{p} 19$ and p16 to Cdk6 push the $\mathrm{N}$ and $\mathrm{C}$ terminal lobes away from each other resulting in deformation of the binding site for the D-type cyclins. In addition, the two inhibitors distort the ATP binding pocket of Cdk6 contributing to further inhibition. 

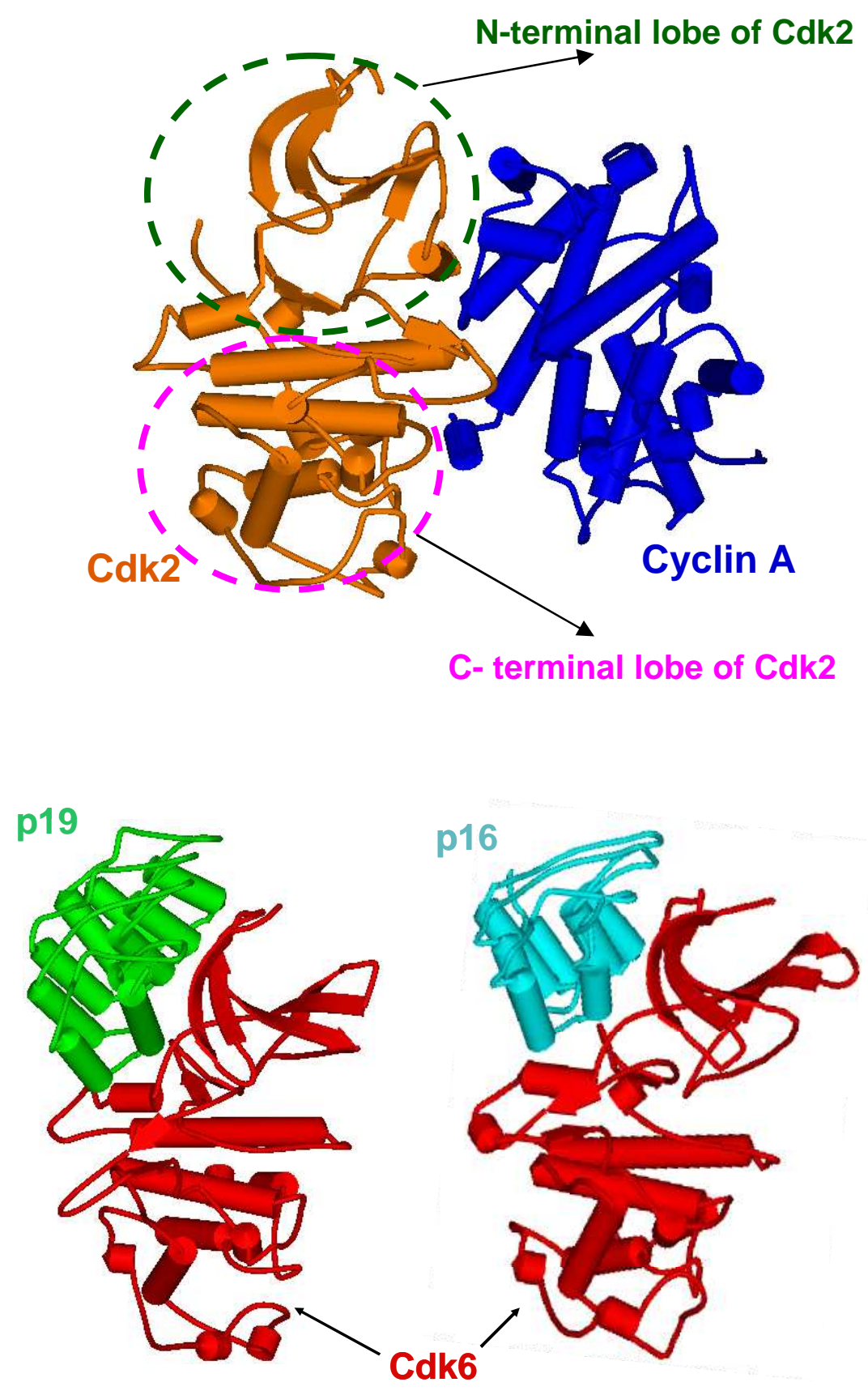

Figure 1.2 Structures of the Cdk2/cyclin A (PDB; 1FIN), p19/Cdk6 (PDB; 1B18), and p16/Cdk6 (PDB; 1B17). The binding of p19 and p16 to the Cdk6 distorts the cyclin binding site preventing the formation of Cdk6/cyclin $D$ complexes. 
On the other hand, members of the Cip/Kip family of CKI's inhibit Cdk catalytic activity by binding to the heterodimeric complex over an extended surface spanning from the cyclin to the Cdk subunit. The Cip/Kip proteins inhibit a broad spectrum of Cdk complexes and have been shown to regulate the activity of Cdk's in complex with cyclins D, E and A. ${ }^{91,92}$ Figure 1.3 shows the versatility of the Cip/Kip proteins in Cdk regulation during the $\mathrm{G} 1 / \mathrm{S}$ transition. Each Cip/Kip protein family member contains an amino terminal domain that has been shown to be necessary and sufficient for Cdk inhibition. This functionally conserved segment is known as the kinase inhibitory domain (KID). The sequences of the KID's of p21 (residues 11 - 94) and p57 (residues 23 - 106) with respect to p27 (residues 22 - 106) are $48 \%$ and $42 \%$ identical. The Cdk regulatory activity of the Cip/Kip proteins causes cell cycle arrest during the G1/S transition. This phase transition is controlled by Cdk1/cyclin A or E, Cdk2/cyclin A or E, Cdk4/cyclin D, and Cdk6/cyclin D. While the inhibition of Cdk1 and Cdk2 complexes by the Cip/Kip proteins is well defined, their effect on Cdk's 4 and 6 is less clear. Early studies on the interaction of the Cip/Kip proteins with Cdk4/cyclin D and Cdk6/cyclin D complexes reported that the Cip/Kip proteins promote assembly of Cdk4/cyclin D and Cdk6/cyclin D complexes, in essence acting as activators. ${ }^{93,94}$ However, reports from other groups have cast doubt on these observations. ${ }^{95,96}$ It should be noted that a mechanism has recently been reported that could possibly explain the apparently paradoxical functions of the Cip/Kip proteins. ${ }^{97}$ The study showed that phosphorylation of p27 by the oncogenic tyrosine kinase, $B C R-A B L$, on a tyrosine residue (Tyr88) that is 


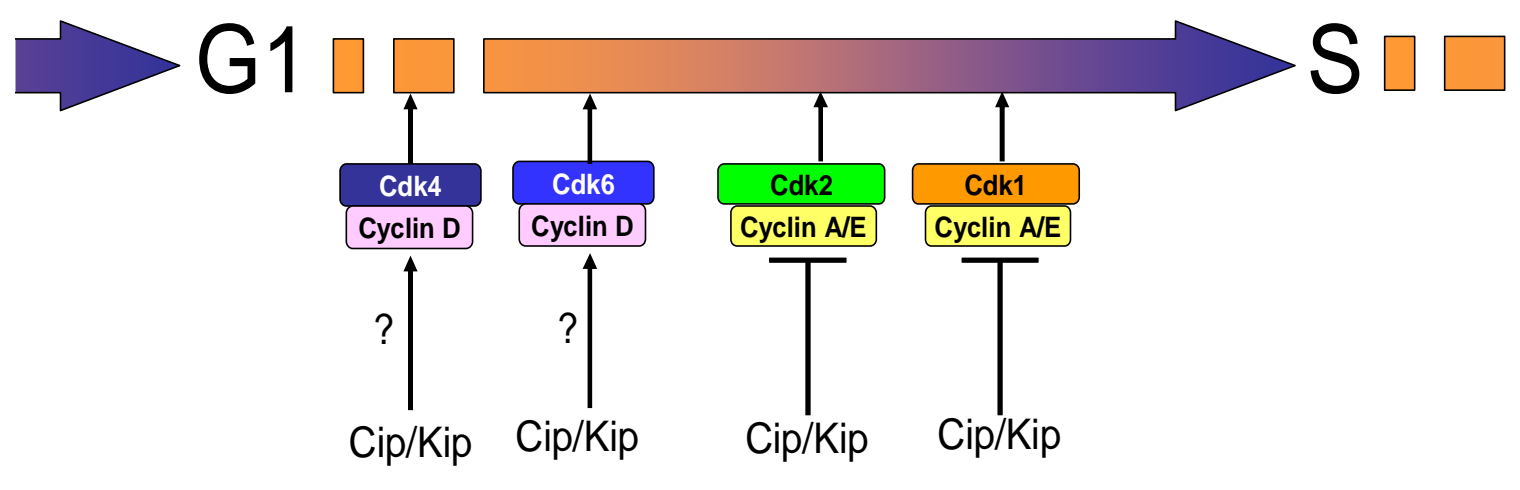

Figure 1.3 The Cip/Kip proteins regulate the activity of a broad range of Cdk/cyclin complexes. 
inserted into the ATP binding pocket during Cdk inhibition, ejects the tyrosine out of the active site, consequently relieving inhibition of kinase activity. The authors propose that this mechanism also takes place in the interaction of p27 and Cdk4/Cdk6 thereby explaining the seemingly paradoxical functions of p27.

The first member of the Cip/Kip family to be identified was p21. p21 inhibits cell proliferation in response to genomic insults and is up-regulated in irradiated cells and in cells exposed to DNA damaging drugs. The up-regulation of p21 is mediated by p53. ${ }^{98,99}$ In contrast to p21, p27 expression is induced by diverse differentiation and anti-mitogenic stimuli including, transforming growth factor- $\beta$ (TGF- $\beta$ ), ${ }^{80,100}$ rapamycin, ${ }^{101}$ contact inhibition, ${ }^{80}$ depletion of growth factors, ${ }^{102}$ and loss of cell anchorage. ${ }^{103}$

The structure of $\mathrm{p} 27$ bound to Cdk2/cyclin A provides detailed insights into the molecular basis of Cdk inhibition by the Cip/Kip proteins. ${ }^{104}$ The KID fragment of p27 was used to prepare the ternary complex. Figure 1.4 shows the structure of the p27-KID/Cdk2/cyclin A complex (PDB accession number, 1JSU). In the complex, p27-KID folds into an extended, non-globular structure consisting of (from $\mathrm{N}$ to $\mathrm{C}$ terminus): an extended segment, an amphipathic $\alpha$-helix, an amphipathic $\beta$-hairpin, a $\beta$-strand and a $3_{10}$ helix. The extended segment (domain D1) binds to the cyclin A subunit of the kinase complex, the amphipathic $\alpha$-helix (domain LH) links the cyclin binding segment to the Cdk binding segment (domain D2) which is comprised of the amphipathic $\beta$-hairpin, the $\beta$-strand, and the $3_{10}$ helix. The extended segment binds to the conserved substrate binding site on the cyclin A subunit. The amphipathic $\alpha$-helix does not make a significant 


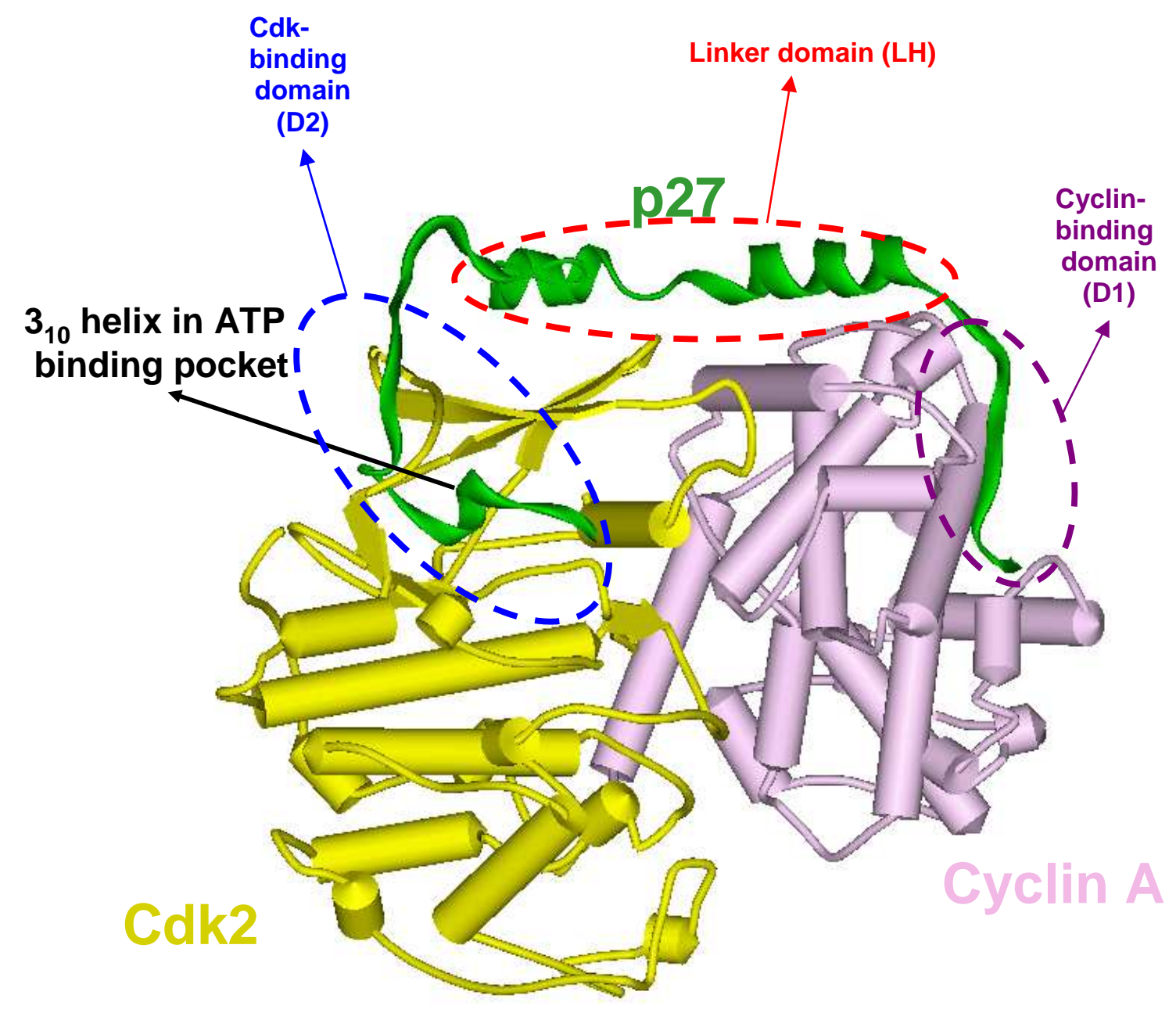

Figure 1.4 The structure of the p27/Cdk2/cyclin A complex (PDB; 1JSU). p27 binds to the Cdk2/cyclin A complex over an extended surface spanning from the cyclin A to the Cdk2 subunit. Binding to cyclin A prevents substrate binding and the binding of the $3_{10}$ helix of p27 into the ATP binding pocket impedes ATP binding. 
number of contacts with the kinase complex. The extended structure of the p27-KID enables it to interact with the Cdk2/cyclin A complex over a large surface area (a total buried surface area of $5,752 \AA^{2}$ ). Most of the hydrophobic residues on p27-KID pack against the Cdk2 and cyclin A subunits. From this structure, the binding of p27-KID inhibits the Cdk kinase activity in three ways. First, the binding of p27-KID to the substrate binding site on the cyclin obstructs the docking of Cdk2 substrates. Secondly, p27-KID binding deforms the catalytic cleft on the Cdk2. This is caused by interactions between p27-KID and an amino-terminal $\beta$-sheet of Cdk2. Finally, the $3_{10}$ helix of p27-KID is inserted into the catalytic cleft preventing ATP binding. ${ }^{104}$ This model of inhibition has been extended to p21 and p57 since the three proteins have very similar primary structures.

\section{3 p27 kinase inhibitory protein $1\left(p 27^{\text {kip1 }}\right)$}

Human p27 is comprised of 198 amino acids and, in addition to cell cycle regulation, has been implicated in regulating differentiation, ${ }^{105,106}$ and more recently the control of cell motility. ${ }^{107}$ p27 is up-regulated in serum-starved and contact inhibited cell cultures resulting in cell cycle exit (quiescence).

Suppression of p27 expression using anti-sense mRNA abrogates cell cycle exit. ${ }^{108}$ When quiescent cultures are supplemented with mitogens, p27 is eliminated from cells and the cells re-enter the cell cycle. ${ }^{109}$ However, quiescent cells do not re-enter the cell cycle, even in the presence of mitogens, when the elimination of p27 is blocked by treatment with cyclic adenosine monophosphate 
(CAMP) analogs, rapamycin, or hypoxia. ${ }^{101,110,111}$ These results demonstrate that p27 is a downstream effector of anti-mitogenic signals. ${ }^{112}$ The in vivo effects of p27 deficiency have been investigated using knock-out mice. Mice lacking p27, though viable, exhibit hyperplasia in multiple organs and gigantism with respect to their wild-type counterparts. ${ }^{113-115}$ As a negative regulator of cell cycle progression, p27 is an oncosuppressive protein. Circumvention of p27 cell cycle control has been linked to a myriad of human malignancies. The loss of function in most tumor suppressor genes is usually a consequence of deletion of one or both alleles of the gene(s). However, the p27 gene is rarely deleted in human cancers. Mouse model studies have shown that p27 is a haploinsufficient tumor suppressor. ${ }^{116}$ Mice with a single allele of the p27 gene have an intermediate frequency of tumor formation relative to their wild-type and null counterparts. ${ }^{116,117}$

\section{4 p27 and cancer}

One of the hallmarks of many types of cancer is the reduced level of p27 protein relative to normal cells. However, this reduction in p27 protein levels does not stem from p27 gene mutations. ${ }^{118}$ In fact, germline mutations in the p27 gene are very rare. ${ }^{119-121}$ Studies of breast carcinoma have revealed that decreased p27 protein levels promote tumor progression and are associated with poor prognosis. ${ }^{122-124}$ The same has also been observed in colorectal, ${ }^{125}$ lung non-small cell, ${ }^{126}$ gastrointestinal, ${ }^{127}$ prostrate, ${ }^{128}$ oral cavity, ${ }^{129}$ and ovarian carcinomas. ${ }^{130}$ In most of these cancers, the p27 mRNA transcript is expressed 
at a normal level indicating that the low level of p27 protein is caused by protein degradation. There are two main ways by which tumor cells evade cell cycle regulation by $\mathrm{p} 27$ : (i) degradation of $\mathrm{p} 27$ at an accelerated rate (cause of reduced p27 protein level) ${ }^{131-133}$ and (ii) sequestration of p27 in the cytoplasm preventing it from inhibiting the nuclear-localized Cdk's. ${ }^{134}$ Like many other cell cycle regulatory proteins, degradation is one of the main ways by which cells regulate p27 levels. The degradation of p27 is controlled in two ways: cytoplasmic ubiquitination by the KPC (Kip1 ubiquitination promoting) complex ${ }^{135}$ and nuclear ubiquitination by the SCF ${ }^{\text {skp2 }}$ complex. ${ }^{136,137,138}$ The two complexes chemically ligate poly-ubiquitin chains to lysine residues on p27. Prior to being polyubiquitinated by the KPC and SCF ${ }^{\text {skp2 }}$ complexes, p27 must be phosphorylated on Ser10 and Thr 187, respectively. After ubiquitination, p27 is degraded by the $26 \mathrm{~S}$ proteosome. ${ }^{139}$ To be recognized by the SCF complex, p27 has to be in complex with either Cdk2/cyclin A or Cdk2/cyclin E. ${ }^{140,141}$ In most malignancies, there are increased levels of the skp2 protein leading to accelerated degradation of p27. ${ }^{142-145}$ Table 1.2 shows different types of cancer and the alterations in the p27 protein. It has also been reported that reduced expression of p27 plays a role in cancer drug resistance. Resistance to docetaxel, one of the most effective chemotherapeutic agents used in breast cancer treatment, has been related to reduced expression of $\mathrm{p} 27 .{ }^{146}$ It is therefore not surprising that designing small molecule inhibitors that mimic p27 inhibitory activity has generated a lot of interest. 


\section{Table 1.2 Alteration of the $\mathrm{p} 27$ protein in human malignancies.}

\begin{tabular}{|c|c|}
\hline Malignancy & Alteration in p27 protein \\
\hline Breast cancer & $\begin{array}{l}\text { Accelerated degradation }{ }^{147} \\
\text { Cytoplasmic localization }^{148}\end{array}$ \\
\hline Prostate cancer & $\begin{array}{l}\text { Accelerated degradation }{ }^{149} \\
\text { Cytoplasmic localization }{ }^{150}\end{array}$ \\
\hline Ovarian cancer & $\begin{array}{l}\text { Accelerated degradation }{ }^{151} \\
\text { Cytoplasmic localization }{ }^{152}\end{array}$ \\
\hline Colorectal cancer & Accelerated degradation ${ }^{153}$ \\
\hline Non-small cell lung cancer & Accelerated degradation ${ }^{145}$ \\
\hline Glioma & Accelerated degradation ${ }^{154}$ \\
\hline Lymphoma & Accelerated degradation ${ }^{155}$ \\
\hline Chronic myelogenous leukemia (CML) & Accelerated degradation ${ }^{133}$ \\
\hline Liver cancer & $\begin{array}{l}\text { Accelarated degradation }{ }^{156} \\
\text { Cytoplasmic localization }{ }^{157}\end{array}$ \\
\hline Oral squamous cell carcinoma & Accelerated degradation ${ }^{143}$ \\
\hline
\end{tabular}




\section{5 p27 is an intrinsically unstructured protein}

The classical structure-function paradigm postulates that proteins must be folded into distinct three-dimensional conformations in order to function. However, there is now sufficient evidence that supports the existence of functional proteins and protein domains that are intrinsically disordered under physiological conditions. ${ }^{158-165}$ As used herein, intrinsic disorder of a protein or protein domain means that under physiological conditions: (i) the isolated protein or protein domain lacks tertiary structure; (ii) there are no significant, long-range interactions between its residues; and (iii) it exists as an ensemble of many highly dynamic conformations. Intrinsic disorder in proteins is either classified as global (disorder of the entire protein), or local (disorder of a protein domain). Proteins that exhibit native structural disorder belong to a fast growing family of proteins referred to either as intrinsically unstructured proteins (IUP's), intrinsically disordered proteins (IDP's), natively unstructured proteins (NUP's), or natively disordered proteins (NDP's). For the purposes of this dissertation, I will use the term intrinsically unstructured proteins (IUP's). It is now estimated that about one third of the human genome is comprised of sequences that encode proteins that have local or global intrinsic structural disorder. Further analyses have shown that the prevalence of IUP's in a particular species varies directly in proportion with its genomic complexity. ${ }^{166}$ The IUP's are involved in key cellular processes including, but not limited to, DNA recognition (DNA binding domains of the GCN4 and NFATC1), ${ }^{167-170}$ transcriptional activation (kinase-inducible activation domain of CREB, activation domains of p53 and VP16), ${ }^{171-175}$ RNA 
recognition (RNA recognition segments of HIV-1 Rev and BIV Tat proteins), ${ }^{176-179}$ translation (elF4E binding segment of elF4G), ${ }^{180}$ microtubule and actin dynamics (caldesmon, thymosin, stathmin/RB3), ${ }^{181-183}$ and cell cycle regulation (p21, p27, p57). Table 1.3 shows a list of some IUP's and their biological functions. IUP's have unique properties that distinguish them from globular proteins.

Relative to globular proteins, the primary structures of IUP's are characterized by low sequence complexity and compositional bias. ${ }^{184}$ They are enriched in amino acids that promote structural disorder such as Ser, Ala, Gly, Arg, Gln, Pro, Glu, and Lys, and relative to globular proteins, exhibit reduced levels of residues that promote structural order such as Trp, Cys, Phe, Ile, Asn, Val, Tyr, and Leu. ${ }^{184,164}$ Globular proteins typically have a comparatively larger number of order promoting hydrophobic amino acids that form their cores and facilitate folding. Bioinformatics programs that predict structural disorder based on the amino acid attributes of the IUP's with a high degree of accuracy have been developed. Examples include PONDR,${ }^{185}$ DISOPRED, ${ }^{186}$ and GLOBPLOT. ${ }^{187}$ The sequence properties of IUP's indicate that their intrinsic disorder is encoded in their primary structures as is the case for the secondary and tertiary structures of globular proteins. Due their intrinsic disorder, IUP's often exhibit extended conformations and have larger hydrodynamic radii relative to globular proteins of comparable mass. ${ }^{163}$ Because of their extended structures, IUP's are sensitive to proteolytic cleavage. ${ }^{188}$ In addition, IUP's are heat stable,${ }^{81}$ have high net charge,${ }^{163}$ and low mean hydrophobicity. ${ }^{163}$ The disordered structures of IUP's have been characterized using X-ray 


\section{Table 1.3 Biological functions of IUP's.}

Protein

Naked2 ${ }^{189}$

Bim, Bad, BMF ${ }^{190}$

C-terminal domain of $\mathrm{CtBP}^{191}$

C-terminal domain of the measles virus nucleoprotein ${ }^{159,192}$

Thymosin- $\beta^{193}$

Acidic activation domain of NF-kB ${ }^{194}$

Basic DNA-binding region of the leucine zipper protein $\mathrm{GCN} 4{ }^{167}$

Amino-terminal domain of $\mathrm{p} 53^{173}$

CITED2 $^{195}$

High mobility group (HMG) domain, of

\section{Function}

\section{Vesicle transport}

Apoptosis

Nuclear transcriptional co-repression, golgi membrane tubule fusion

Measles virus RNA synthesis

Actin dynamics

Transcriptional activation

Transcriptional activation

Onco-suppressing transcription factor

Hypoxic response

Regulation of the T-cell receptor-a gene enhancer 
crystallography, nuclear magnetic resonance (NMR), circular dichroism (CD) spectroscopy, raman optical activity spectroscopy (ROA), fourier-transformed infra-red spectroscopy (FTIR), analytical ultracentrifugation (AUC), dynamic light scattering (DLS), small angle X-ray scattering (SAXS), and fluorescence spectroscopy. Although IUP's have disordered structures prior to binding their targets, NMR and CD studies have demonstrated that some IUP's have segments that exhibit a natural propensity for nascent secondary structures. ${ }^{197,198,188,199}$ One key property of most IUP's is that they fold on binding their targets and become ordered. ${ }^{197,175,200}$ This folding-on-binding phenomenon was manifested in the interaction of p27 and Cdk2/cyclin A complex. ${ }^{197}$

p27 is a globally unstructured IUP with segments that exhibit nascent secondary structure. Of the three Cip/Kip proteins, p27 is the best characterized structurally because it can be expressed at high levels in E. coli, can be purified to homogeneity, and is highly soluble. p27 exhibits many characteristics of IUP's. Consistent with the properties of IUP's, full-length p27 migrates at about $27 \mathrm{kDa}$ during a sodium dodecyl sulfate polyacrylamide gel electrophoresis (the calculated molecular weight of p27 is $22 \mathrm{kDa}$ ) indicating that it is a highly extended polypeptide with a large hydrodynamic radius. ${ }^{80}$ Moreover, analysis of p27 by limited proteolysis shows that free p27 is very susceptible to cleavage. ${ }^{201}$ However, when p27 interacts with the Cdk2/cyclin A complex, it is refractory to proteolysis. ${ }^{201}$ The secondary structures of p27, p21 and p57 determined by CD at low concentrations are strikingly similar. ${ }^{188,198,202}$ The three proteins are 
largely unstructured with a low population of helical secondary structure. The CD spectra show that the native structural disorder of the Cip/Kip proteins is well conserved. It should be noted that the results on secondary structure discussed above are derived from studies of KID constructs and not the full-length proteins. Like the KID's, the full-length proteins are also largely unstructured. NMR analysis shows that full-length p27 and p27-KID have very similar secondary structures. ${ }^{197}$ The conservation of the disordered structures of the Cip/Kip proteins argues strongly that structural disorder plays a significant role biological function. p27 folds dramatically on binding the Cdk2/cyclin A complex and attains structural order as shown in Figure 1.4, above. The plasticity of the structure of p27 and its template driven folding on binding enables p27 to interact with a broad range of Cdk complexes. ${ }^{203}$ The folding on binding mechanism affords high specificity but at a great entropic cost. It therefore follows that the thermodynamic contributions of the binding-on-folding process due to enthalpy and entropy have to be appropriately balanced so that binding occurs spontaneously.

\subsection{Thermodynamics of the association of p27 and Cdk2/cyclin A}

The KID's of the Cip/Kip proteins are comprised of three domains: (i) a cyclin-binding domain (hereafter referred to as D1); a linker domain (hereafter referred to as $\mathrm{LH}$ ); and (iii) a Cdk binding domain (hereafter referred to as D2).

D1 has a motif that binds to the substrate binding site on the cyclin subunit that is comprised of Leu32, Phe33, and Gly34. These three residues are conserved 
amongst the Cip/Kip proteins. On D2, one key residue is Tyr88 which is inserted into the ATP binding pocket when p27 interacts with the Cdk2/cyclin A complex. Tyr88 is part of the $3_{10}$ helix of p27 that was discussed in Section 1.2. Figure 1.5 shows a sequence alignment of the KID's of members of the Cip/Kip family. The sequences D1 and D2 are well conserved amongst the three family members, but the sequence of domain LH is not conserved. Only 3 of the 22 residues in this domain are conserved. The number of residues in domain $\mathrm{LH}, 22$, is the same for all the Cip/Kip proteins. As discussed above, the secondary structures of the Cip/Kip proteins are mainly unstructured with nascent helical properties. Nuclear magnetic resonance (NMR) ${ }^{13} \mathrm{C}$-alpha $\left({ }^{13} \mathrm{C} \alpha\right)$ chemical shift analysis showed that D1 and D2 are largely unstructured and the nascent helical structure observed using CD is localized in the LH domain. ${ }^{197}$ The nascent helical structure of the linker domain is conserved amongst all three members of the family. ${ }^{188,198,202}$ This sequence divergence/structure conservation relationship is atypical since the domains whose sequences are conserved, D1 and D2, are unstructured, and the domain whose sequence is poorly conserved, LH, exhibits conserved structural features.

Thermodynamically, the folding of p27 on binding the Cdk2/cyclin A is very unfavorable since it requires a large negative entropy change. Lacy, et al., demonstrated that this large negative entropic cost is overcome by the quantity of heat (enthalpy associated with binding, $\Delta \mathrm{H}$ ) released during the extensive folding of p27 on binding to Cdk2/cyclin A. ${ }^{197}$ This results in a negative Gibbs free energy change $(\Delta G)$ for binding and spontaneous binding. The interaction of p27 


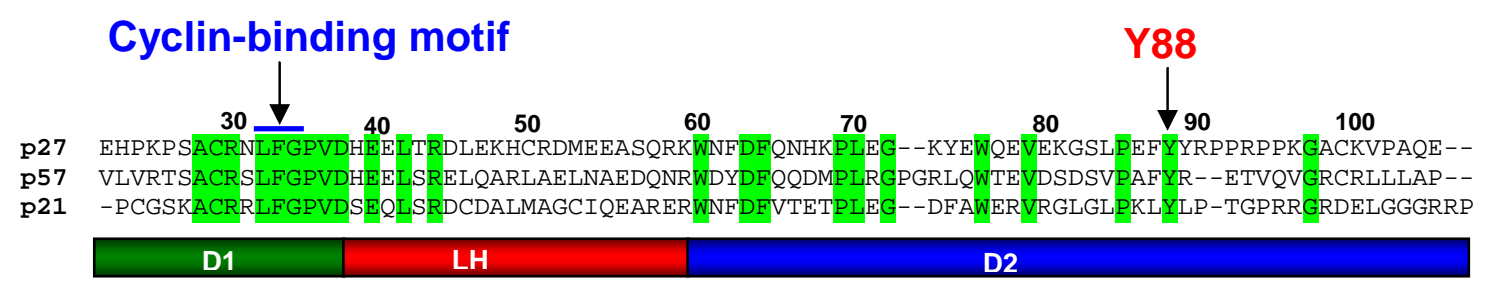

Figure 1.5 Sequence alignment of the KID domains of the Cip/Kip proteins. 
and the Cdk2/cyclin A complex is therefore an enthalpically driven process. The heat capacity change associated with binding $\left(\Delta \mathrm{C}_{p}\right)$ was found to be large and negative, and comparable to values obtained from protein folding events. The large negative heat capacity change is an indicator of the extent to which

hydrophobic interactions contribute to the formation of the ternary complex. ${ }^{204,205}$ Lacy, et al, further demonstrated that the interaction of p27 with the Cdk2/cyclin A complex is more favorable than the interaction of p27 with either cyclin A or Cdk2 alone. The binding of p27 to the Cdk2/cyclin A complex is sequential, with p27 binding to the cyclin A subunit first, followed by folding of the linker domain, and finally the folding of D2 into a $\beta$-hairpin, a $\beta$-strand and a $3_{10}$ helix. The kinetics of association and dissociation of p27 and cyclin A are much faster relative to those of p27 and Cdk2. While the functions of D1 and D2 are well characterized, the role of domain LH is not well understood. Is it just a spacer between $\mathrm{D} 1$ and $\mathrm{D} 2$ or does it play a role in the binding process? If so, why does it have a conserved structure?

\subsection{Project hypotheses and strategy}

To understand the role of the linker domain of p27, I first investigated the interactions of D1 and D2 with P-Cdk2/cyclin A (a mutant with the linker domain deleted). Given the structural conservation of the linker domain of p27, I hypothesize that linker domain is necessary for the interaction of p27 and the P-Cdk2/cyclin A complex and for p27 to perform its biological function. 
Although the sequence of domain $\mathrm{LH}$ is not conserved amongst members of the Cip/Kip protein family, it is very highly conserved for each protein in different species. Figure 1.6 shows an alignment of the sequences of p27 LH domains in different species. In mammals (from mouse to man - a period of over 100 million years in the evolutionary timeline), the sequence of the p27 linker domain is $100 \%$ conserved and has resisted selective pressure. This indicates that the sequence of each individual protein may be significant in determining its functional properties. Otherwise, there would have been no reason for these sequences to be selected for and to persist during evolution. However, the evolutionary stability of these sequences raises a question as to whether it is the sequence or the structure of the LH domain that is important in determining the biophysical and functional properties of these proteins. To answer this question, I designed p27-KID mutants in which the LH domain of p27 was replaced with the LH domains of either p21 (p27-KID ${ }^{p 21 \text { helix }}$ ) or p57 (p27-KID $\left.{ }^{p 57 h e l i x}\right)$ and studied their interaction with the Cdk2/cyclin A complex. These mutants are hereafter referred to as the chimeric mutants. Since D1 and D2 are well conserved in the three proteins, the chimeric mutants should be suitable for studying the sequence-divergence/structure-conservation relationship of the linker domains of the three Cip/Kip proteins. I hypothesize that if the structure of the LH domain is the dominant determinant of the biophysical and functional properties of the Cip/Kip proteins on interacting with the Cdk2/cyclin A complex, then the properties of the $\mathrm{p} 27-\mathrm{KID}^{\mathrm{wt}}$ relative to the chimeric mutants would be very similar (recall that amongst the Cip/Kip proteins, the structures are conserved but the 


\begin{tabular}{lll}
\multicolumn{1}{l}{ Frog } & HDELRSELKRQLKEIQASDCQR & 32 \\
\hline Pig & HEELTRDLEKHCRDMEEASQRK & 37 \\
Cat & HEELTRDLEKHCRDMEEASQRK & 37 \\
Mink & HEELTRDLEKHRRDMEEASQRK & 37 \\
Human & HEELTRDLEKHCRDMEEASQRK & 37 \\
Hampster & HEELTRDLEKHCRDMEEASQRK & 37 \\
Rat & HEELTRDLEKHCRDMEEASQRK & 37 \\
Mouse & HEELTRDLEKHCRDMEEASQRK & 37 \\
\hline Chickhen & HEELNRDLKKHRKEMEEACQRK & 37 \\
Zebrafish & HEELKKDFQRQLRTMEDASAEA & 37
\end{tabular}

Figure 1.6 Alignment of p27-LH domains from different species. The sequences of LH domains from mammals are circumscribed in the red box. 
sequences are different). On the other hand, if the sequence of the LH domain is the dominant determinant of the properties of the interaction between the Cip/Kip proteins and P-Cdk2/cyclin A, then the properties of the interaction of the chimeric mutants with $\mathrm{P}-\mathrm{Cdk} 2 /$ cyclin A relative to $\mathrm{p} 27-\mathrm{KID}^{\mathrm{wt}}$ would be different. In addition to the sequence-structure relationship of the linker domains of the Cip/Kip proteins, I also studied the role of the three conserved residues in domain LH by mutating the residues to glycines (Glu39Gly/Leu41Gly/Arg43Gly, the p27-KID ${ }^{\Delta C}$ mutant) and probed the interaction of this mutant with the PCdk2/cyclin A complex.

Many IUP's exhibit a small amount of nascent helical secondary structure in the absence of their binding partners. The polypeptide segments giving rise to these features typically fold into $\alpha$-helices upon binding their biological targets. I propose that one of the roles of the nascent helical structures is to attenuate the entropic cost associated with the folding-on-binding process. I further hypothesize that increasing the helical content of the linker domain would decrease the entropic cost associated with the folding on binding and thereby decrease the Gibbs free energy of interaction of the p27-KID and a Cdk/cyclin complex. Conversely, decreasing the helical content would increase the entropic cost and make the reaction less favorable. One underlying assumption of these hypotheses is that all the mutants fold in exactly the same way as the wild-type protein. To test these hypotheses, p27-KID mutants which have LH domains that are either more $\left(\mathrm{p} 27-\mathrm{KID}^{+\mathrm{H}}\right)$ or less $\left(\mathrm{p} 27-\mathrm{KID}^{-\mathrm{H}}\right)$ helical relative to the wild-type p27-KID (p27-KID ${ }^{\mathrm{wt}}$ ) were designed and their thermodynamic and functional 
properties on binding to the Cdk2/cyclin A complex probed. In addition, I also designed a mutant in which the LH domain of p27 was replaced with a flexible loop, 22 residues long, and performed similar studies. 


\section{Chapter 2: Materials and methods}

\subsection{Design of the more and less helical p27-KID linker domain mutants}

My approach of designing p27-KID mutants with linker domains that were either more or less helical than the wild-type p27-KID was to substitute solvent exposed residues within the linker domain with residues that are known to either stabilize or destabilize helices. Mutating the solvent exposed residues minimizes disruption of contacts between p27 and the Cdk2/cyclin A complex. This reduces the possibility that the results obtained from the succeeding studies are a consequence of the disruption of contacts between p27 and Cdk2/cyclin A. The solvent accessible surface area (SASA) for each residue within the linker domain was analyzed using the GETAREA program. ${ }^{206}$ The input used for the program was the pdb file of the p27-KID/Cdk2/cyclin A complex (1JSU). Residues that had more than $50 \%$ of their total surface area exposed to solvent were selected as suitable candidates for mutagenesis. From the output, 10 residues had more than $50 \%$ of their surface areas exposed (Table 2.1). The 10 candidate residues were further analyzed using the Insight II program (Accelrys, San Diego, CA). The PDB file, 1JSU, was used as an input and all the atoms within a $6 \AA$ radius of every atom of each candidate residue were analyzed. This was done to determine whether any of the candidate residues interacted with the residues of the binary complex. No close contacts were observed for the 10 candidate residues. After this analysis, all the 10 candidate residues were determined to be suitable for mutagenesis. Figure 2.1 shows the orientation of the side chains of 
Table 2.1 Solvent exposure of the residues in the linker domain of p27.

\begin{tabular}{lc}
\hline Residue & Percentage of surface area exposed to solvent \\
\hline His38 & 40.9 \\
Glu39 & 84.1 \\
Glu40 & 79.9 \\
Leu41 & 1.9 \\
Thr42 & 22.7 \\
Arg43 & 56.7 \\
Asp44 & 33 \\
Leu45 & 0.4 \\
Glu46 & 26.9 \\
Lys47 & 76.2 \\
His48 & 27.2 \\
Cys49 & 13.2 \\
Arg50 & 67.1 \\
Asp51 & $\mathbf{5 5 . 4}$ \\
Met52 & 20.2 \\
Glu53 & 37.9 \\
Glu54 & $\mathbf{8 1 . 6}$ \\
Ala55 & $\mathbf{8 7 . 1}$ \\
Ser56 & 14.6 \\
Gln57 & 30 \\
Arg58 & \\
Lys59 & 37 \\
\hline & \\
\hline
\end{tabular}

The residues selected for mutagenesis are in bold font. 


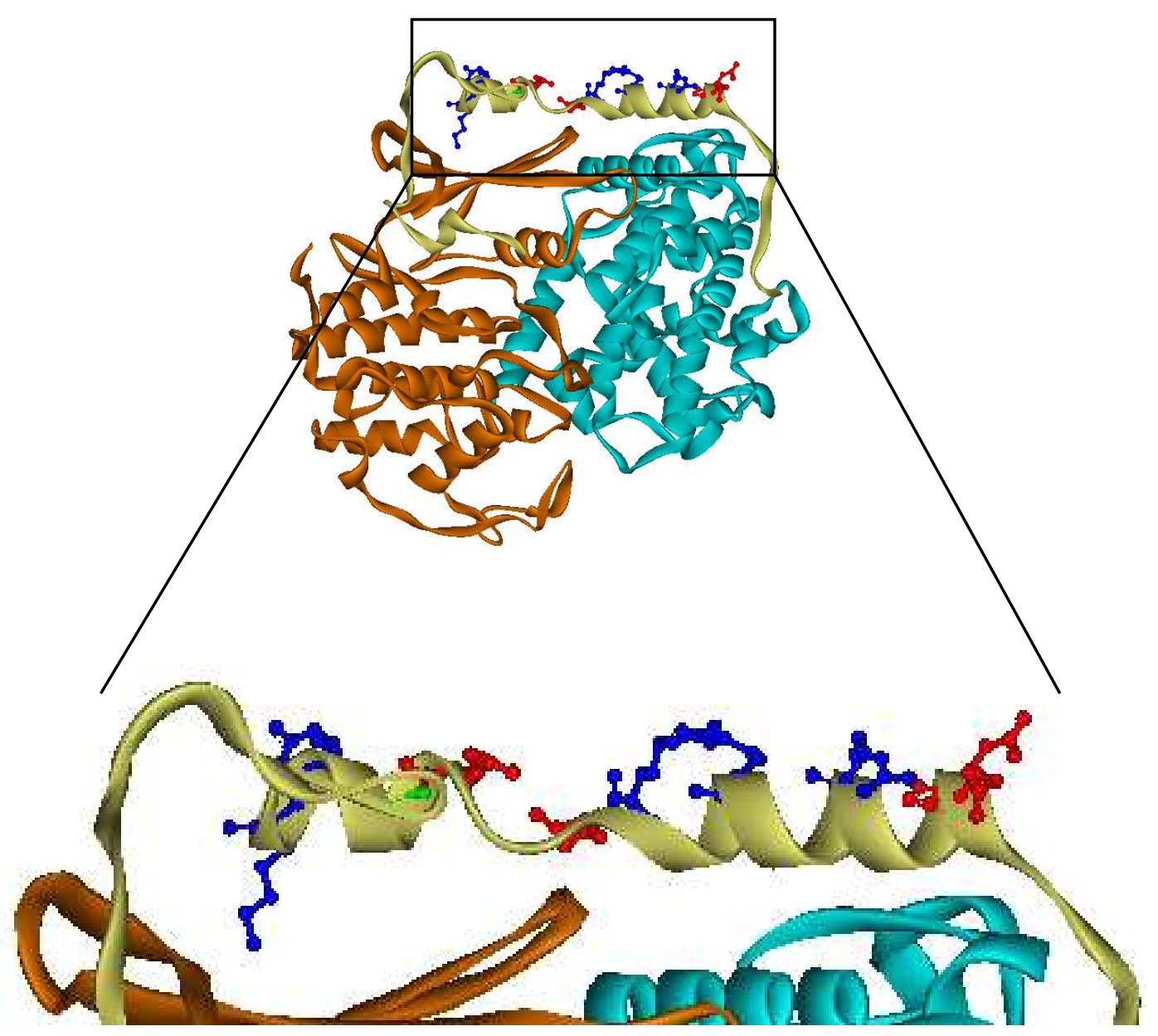

Figure 2.1 The structure of p27/Cdk2/cyclin A (PDB; 1JSU) showing the residues determined to be suitable for mutagenesis. The residues are colored red for acidic residues, blue for basic residues, and green for hydrophobic residues. Cdk2 is colored orange and cyclin A cyan. 
the selected residues in the structure of the p27/Cdk2/cyclin A structure (PDB; 1JSU).

The stability an $\alpha$-helix is determined both by the helix forming propensity of the amino acids in its primary structure and the interactions between side chains. ${ }^{207}$ Two different strategies were used in the design of mutants with stabilized linker domains. In the first approach, alanine was used as a possible stabilizer of the helical structure of the linker domain. Two alanine mutants were designed. In the first alanine mutant, all the residues that were selected for mutagenesis were substituted with alanines. Alanine was chosen because previous studies have shown that alanines stabilize and have a high propensity to occur in $\alpha$-helices. ${ }^{208-210}$ This mutant, in which the 10 candidate residues were substituted with alanines, was designated $\mathrm{p} 27-\mathrm{KID}^{\mathrm{A} 10}$. In the second alanine mutant, all the 22 residues in the linker domain were substituted with alanines. This mutant was designated p27-KID ${ }^{\mathrm{A} 22}$. In the second approach, leucine was used as a potential stabilizer of the linker domain helix. Like alanine, leucine has also been shown to stabilize and have a high propensity to occur in $\alpha$-helices. ${ }^{207,209}$ Hydrophobic interactions between leucine side chains on one face of an $\alpha$-helix shield the backbone amides and carboxyl groups from hydrogen bonding with water, thus stabilizing the helical conformation. ${ }^{211}$ One disadvantage of leucine, however, is its hydrophobic nature. To counter the hydrophobicity of leucine, I designed a library of mutants in which the number of leucines replacing the candidate residues was balanced with charged, polar residues. The substituted residues in these mutants consisted of either 7 
leucines and 3 polar residues, 6 leucines and 4 polar residues, 5 leucines and 5 polar residues, etc. I also ensured that the polar residues had a high helical propensity. The leucines were strategically positioned three and four residues apart in the helix. Past studies have shown that leucines positioned three and four residues apart stabilize $\alpha$-helices. ${ }^{212}$ In addition, leucines positioned four residues apart is one of the most commonly observed pairwise side chain interactions in protein $\alpha$-helices. ${ }^{213}$ A total of 6 mutants were designed. The mutants were designated p27-KID ${ }^{S L 1}$ (SL1; stabilized by leucine, construct 1), p27-KID ${ }^{S L 2}, p 27-K_{I}{ }^{S L 3}\left(p 27-K I D^{+H}\right), p 27-K^{S I D}{ }^{S L 4}, p 27-K I D^{S L 5}$, and p27-KID ${ }^{S L 6}$. In the $\mathrm{p} 27-\mathrm{KID}^{\mathrm{SL6}}$ mutant, all the candidate residues were substituted with leucines. This mutant was designed to test if charged residues that were determined to be unsuitable for mutagenesis could counter the hydrophobicity of the 10 leucines.

For the less helical mutant, the candidate residues were replaced with glutamate (Glu) residues. The premise behind choosing Glu residues is that electrostatic repulsion between the negatively charged Glu side-chains should destabilize the helix backbone. ${ }^{214}$ In addition, the substituting residues close to the carboxy terminus of the helix with Glu residues would result in further destabilization of the helix due to repulsion of the negatively charged Glu side-chain and the negative carboxy-terminus helix dipole. ${ }^{215}$ Another advantage of using glutamate is that the polar Glu side-chain should enhance the solubility of this mutant. This mutant was designated $\mathrm{p} 27-\mathrm{KID}^{-\mathrm{H}}$.

A mutant with a flexible loop comprised of mainly glycines, a known helix breaker, and other residues with a low propensity for being in $\alpha$-helices was also 
designed. The residues that comprised this mutant were chosen with an objective of minimizing helical secondary structure in the linker domain. The number of residues in the loop equaled 22, as in the p27 linker domain. This mutant was designated p27-KID ${ }^{\text {loop }}$. Table 2.2 shows the linker domain sequences of all the mutants designed.

\subsection{Preparation of mutagenesis cassettes}

The p27-KID linker domain mutants were cloned by cassette mutagenesis. First, the deoxyribonucleic acid (DNA) sequence encoding residues 22-71 of p27 (including the cyclin binding domain, the linker domain, and the Cdk binding domain up to E71) was determined. The bases encoding the wild-type p27 linker domain residues were changed such that they encoded the linker domain sequences of the mutants. Oligonucletides used in the preparation of the mutagenesis cassettes were then designed. For each mutant, a forward and reverse oligonucleotide, each 104 bases long, with 40 overlapping complementary bases and end primers for each oligonucleotide, were designed. The sequences of the oligonucleotides used for the preparation of the mutagenesis cassettes are shown on Table A.1. End primers with the sequences 5' GTAACGCATATGGAGCACCCCAAGCCCTCGGCC 3' and 5' CCCCGCCTCGAGGGGTTTGTGATTCTGAAAATCGA 3' were designed for the p27-KID ${ }^{\text {p21helix }}$ and $\mathrm{p} 27-\mathrm{KID}^{\mathrm{p} 57 \text { helix }}$ mutants. The sequences for the end primers designed for the other mutants were: 5' GCCTCCCATATGGAGCAC-CCCAAG 3' and 5' CTTGGGCTCGAGGGGTTTGTGATTC 3'. Included in the 
Table 2.2 Linker domain sequences for the p27-KID variants designed to be more and less helical.

\begin{tabular}{|c|c|}
\hline Variant & Sequence of linker domain \\
\hline $\mathrm{p} 27-\mathrm{KID}^{\mathrm{wt}}$ & 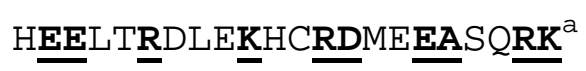 \\
\hline $\mathrm{p} 27-\mathrm{KID}^{\mathrm{A} 10}$ & HAALTADLEAHCAAMEAASQAA $\underline{A}^{\mathrm{b}}$ \\
\hline $\mathrm{p} 27-\mathrm{KID}^{\mathrm{A} 22}$ & AAAAAAAAAAAAAAAAAAAAAA \\
\hline $\mathrm{p} 27-\mathrm{KID}^{\mathrm{SL} 1}$ & HLELTLDLELHCLLMELLSQRK \\
\hline p27-KID ${ }^{S L 2}$ & HEELTLDLELHCLLMELLSQRK \\
\hline p27-KID ${ }^{S L 3}\left(p 27-K D^{+H}\right)$ & HEELTKDLELHCLLMELLSQRK ${ }^{\mathrm{b}}$ \\
\hline p27-KID ${ }^{\text {SL4 }}$ & HEELTKDLELHCKLMEELSQKKK \\
\hline $\mathrm{p} 27-\mathrm{KID}^{\mathrm{SL} 5}$ & HLELTLDLEEHCLKMELESQLK $\underline{L}^{\mathrm{b}}$ \\
\hline $\mathrm{p} 27-\mathrm{KID}^{\mathrm{SL6}}$ & HLLLTLDLELHCLLMELASQLL $\underline{\underline{L}} \underline{L}^{\mathrm{b}}$ \\
\hline $\mathrm{p} 27-\mathrm{KID}^{-\mathrm{H}}$ & HEELTEDLEEHCEEMEEESQEE $\underline{E}^{\mathrm{b}}$ \\
\hline p27-KID ${ }^{\text {loop }}$ & TNP GNGGHGGTGVGGNGGNGHG ${ }^{\mathrm{b}}$ \\
\hline
\end{tabular}

${ }^{a}$ The residues determined to be suitable for mutagenesis are underlined and are in bold font in the linker domain sequence of the wild-type p27-KID (p27-KID ${ }^{\text {wt }}$ ).

${ }^{\mathrm{b}}$ The mutated residues are underlined. 
oligonucleotide design, were a 5' Ndel restriction site and a 3' Xhol restriction site for all the mutants. The mutagenesis cassettes were prepared by overlap extension polymerase chain reaction (oePCR). The overalap extension polymerase chain reaction is illustrated in Figure A.1. First, the single stranded non-overlapped ends of the long oligonucleotides are filled in by a proofreading polymerase (Expand high fidelity polymerase, Roche, Mannhein, Germany) to yield a double stranded DNA cassette. However, this double stranded DNA cassette product is in low quantities since only a small amount (10 ng) is added to the reaction. In the succeeding cycles, the end primers, which are present in large quantities in the reaction (125 ng), amplify the double stranded DNA cassettes. The details of the oePCR reaction that I performed including concentrations of reactants, and thermocycler settings are reported in Table A.2 and Table A.3. Figure 2.2 shows the product from the oePCR reaction for the preparation of the mutagenesis cassette for the $\mathrm{p} 27-\mathrm{KID}^{-\mathrm{H}}$ mutant. Similar product quantities were obtained for the other mutants.

\subsection{Preparation of cassettes for cloning Cip/Kip linker domain peptides}

Overlap extension PCR was also used in the preparation of oligonucleotide cassettes that were used to prepare expression plasmids for Cip/Kip linker domain peptides. For the preparation of each peptide cassette, two long oligonucleotides (98 bases each; 37 overlapping complementary bases) were designed and end primers were designed. Table A.4 shows the sequences of the oligonucleotides and end primers that were designed for each linker 


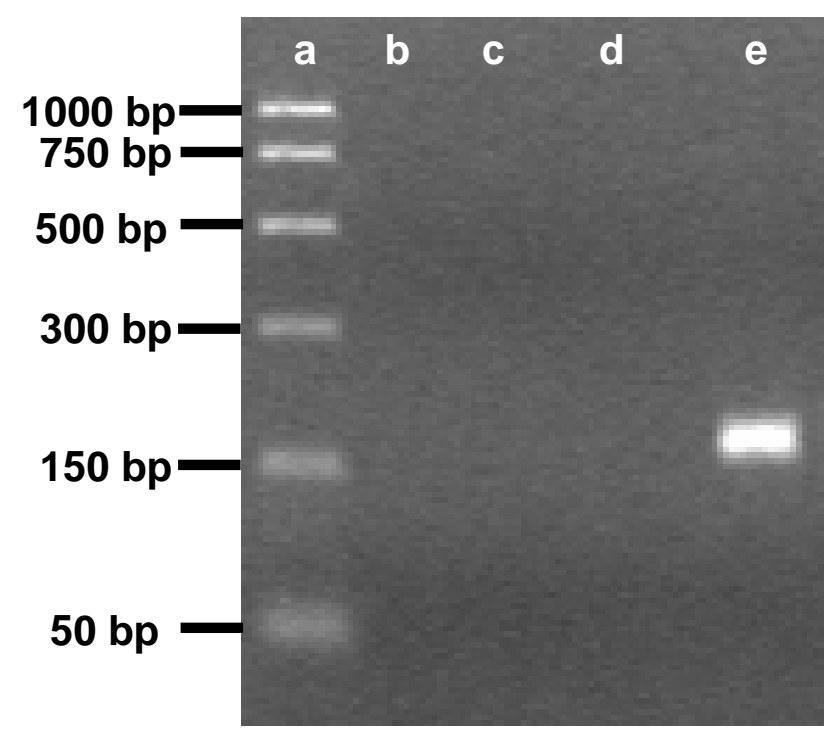

Figure 2.2 Agarose gel analysis of the oePCR for the p27-KID ${ }^{-\mathrm{H}}$ mutant. The descriptions of the lanes are as follows: (a) PCR markers, (b) No primer control, (c) No enzyme control, (d) No template control, and (e) p27-KID ${ }^{-H}$ oePCR. The expected size of the $\mathrm{p} 27-\mathrm{KID}^{-\mathrm{H}}$ oePCR product is $168 \mathrm{bp}$. 
domain peptide. The cassette design for each peptide included (in the 5' to $3^{\prime}$ direction with respect to the coding strand): (i) a 5' Sacll restriction enzyme site, (ii) bases encoding a 6-histidine (His) tag, a thrombin cleavage site, the linker domain sequence, and a stop codon, and (iii) a 3' EcoRI restriction site. The Cip/Kip linker domain sequences designed included a Trp residue after the 22nd residue of the linker domain to facilitate protein concentration determination since these peptides otherwise lack chromophores that absorb light near $280 \mathrm{~nm}$. It should be noted that there is a conserved Trp at the end of the Cip/Kip linker domain sequences (corresponding to Trp60 of p27). The cloning cassette for each peptide was produced in high yield using this procedure. The oePCR product for the p27 linker domain peptide is shown on Figure A.2. The concentrations of the reagents and the thermocycler settings were the same as in Section 2.2 above.

\subsection{Preparation of Escherichia coli expression plasmids}

The pET28a (+) plasmid (Novagen, San Diego, CA) was used as the starting vector to construct plasmids for p27 protein expression in Escherichia coli (E.coli). The multi-cloning site of the pET28 vector is shown on Figure 2.3. Three sets of plasmids were prepared.

The first set of plasmids prepared was used for cloning the Cip/Kip linker domain peptides. The starting plasmid was the $\mathrm{pET} 28$ vector. A cassette encoding a glutathione S-transferase tag with 5' Ncol site and 3' EcoRI restriction sites was cloned between the $\mathrm{Ncol}$ and $\mathrm{EcoRI}$ restriction sites. It should be 


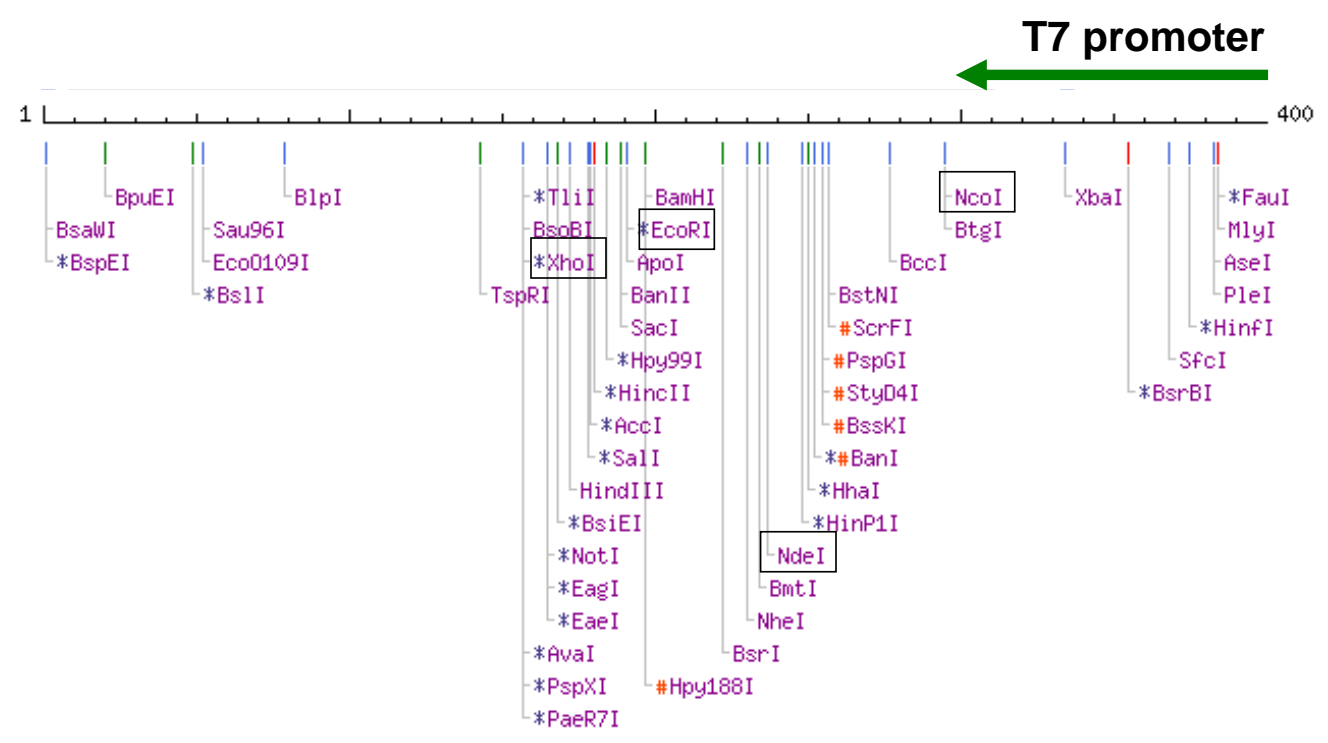

Figure 2.3. The muticloning site of the pET28 vector. The key restriction sites are boxed in rectangles. 
noted that there is a Sacll site that was engineered into the cassette between the end of the GST sequence and the EcoRI site. The cassettes for the Cip/Kip linker domain peptides prepared in Section 2.3 were digested with Sacll and Ndel and cloned into the vector. As mentioned in Section 2.3 above, the Cip/Kip linker domain peptides have a His tag at their 5' ends after the Sacll restriction site. The plasmids formed after ligation of the Cip/Kip linker domain cassettes into the pET28-GST-His vector therefore have both GST and His tags. This vector is hereafter referred to as the pET28-GST-His vector. Cip/Kip linker domain peptides with both GST and His tags have two advantages. First, it provides more purification options. In addition, the use of a GST-His tag makes the fusion protein large enough to be visualized using sodium dodecyl sulfatepolyacrylamide gel electrophoresis (SDS-PAGE). Without the large GST tag, the small peptides (about $3.3 \mathrm{kDa}$ each) would easily diffuse out of standard concentration polyacrylamide gels. Figure 2.4 shows a diagram of the pET28-GST-His vector used to express the p27 linker domain peptide.

The second set of plasmids prepared was used to express the p27-KID mutants. A pET28 plasmid with p27-KID cloned between the Ndel and EcoRI sites had previously been prepared in the laboratory. ${ }^{197}$ The sequence of the p27-KID coding region is shown on Figure A.3. The Xhol site that follows the EcoRI site in the pET28 vector was mutated to prevent restriction by the Xhol enzyme. Within the p27 Cdk binding domain (D2) sequence, the bases encoding Leu70 and Glu71 were mutated from CTAGAG to CTGGAG (the Xhol site). The cassettes of the p27-KID mutants prepared in Section 2.2 were then digested 


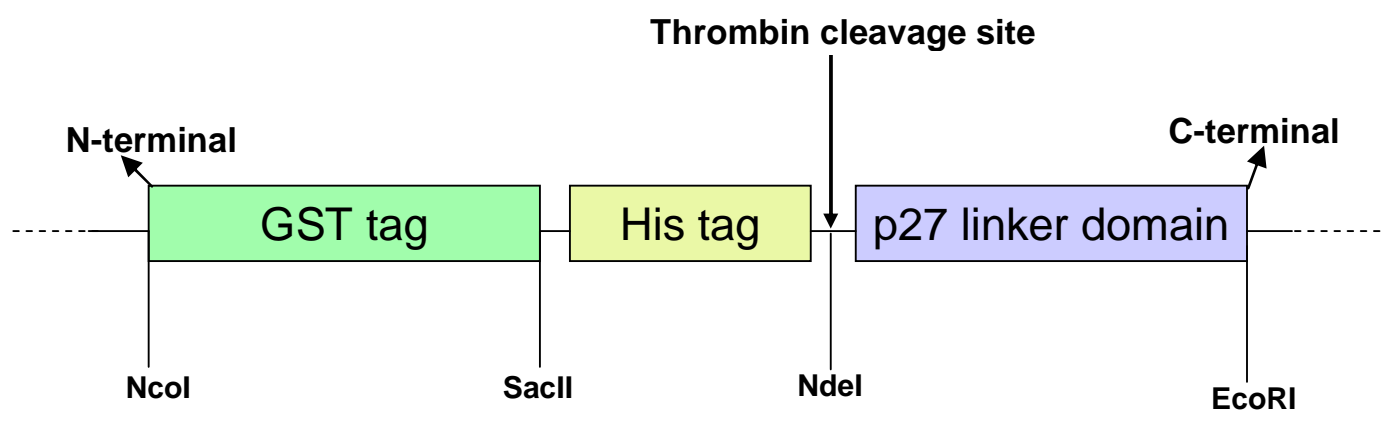

Figure 2.4 The pET28-GST-His p27-LH vector construct. 
with Ndel and Xhol restriction enzymes and ligated into the pET28-p27-KID vector with the engineered Xhol site. Some of the cassettes for the p27-KID mutants were ligated into the pET28-GST-His vector. A cassette coding for p27-KID was amplified from the pET28p27-KID vector by PCR using the following primers: 5' GCGGCCTGGTGCCGCGCGGCAGCCAT 3' and 5' CTTGTCGACG-GAGCTCGAATTCGGA 3'. The cassette and the pET28-GST-His-p27-LH plasmid were both digested using Ndel and EcoRI enzymes followed by ligating the p27-KID cassette into the digested pET28-GST-His vector. This plasmid was termed pET28-GST-Hisp27-KID. The cassettes for the p27-KID mutants were ligated into the p27-KID coding region of the pET28-GST-Hisp27-KID by cassette mutagenesis in the same way that they were cloned into the pET28p27-KID plasmid. Table A.5 shows the p27-KID mutants and the plasmids that they were cloned into. Figure 2.5 shows a schematic diagram of the cassette mutagenesis design.

The last set of plasmids prepared was used for ligating mutants into a pET28vector with the full-length p27 (flp27) cloned between the Ndel and EcoRI sites. The sequence of the flp27 coding region of this vector is shown in Figure A.4. The DNA sequence encoding flp27 has a Pstl restriction site (CTGCAG) within the bases coding for Ala28, Cys29, and Arg30 (GCCTGCAGG). These residues are located in the cyclin binding domain (D1) of p27. In addition, bases coding for the residues Pro92 and Arg93 constitute a Sacll restriction enzyme recognition site (CCGCGG). These residues lie within the Cdk binding domain of p27. The pET28a (+) plasmid does not have Pstl or Sacll restriction sites. The 
Cassette preparation Preparation of the pET28-GST-His vector

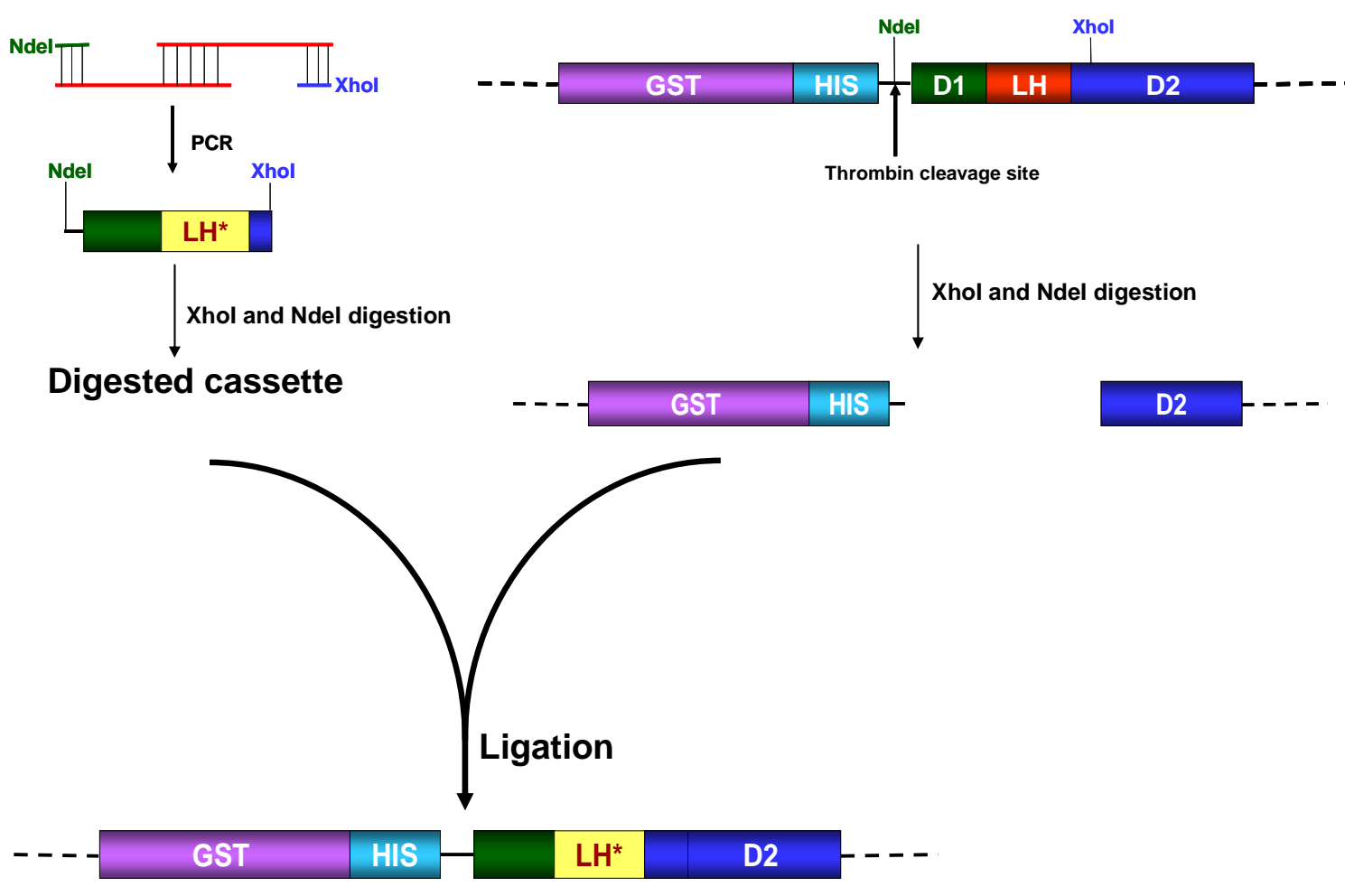

Figure 2.5 Schematic diagram of the cassette mutagenesis design. $\mathrm{LH}^{*}$ is a mutated cassette of a p27-KID linker domain variant. The same approach was used to clone the mutant cassettes into the pET28p27-KID vector. 
following primers were used to amplify cassettes from the plasmids in which the p27-KID linker domain mutants had been cloned: 5' GCCCTCGGCCTGCAGGAACCTCTTCGGCCCGG 3' and 5' CAGGCACCTTTGGGGGGCCGCGGGGGTC 3'. The pET28p27-KID ${ }^{S L 3}\left(p E T 28 p 27-K^{\prime} D^{+H}\right), p E T 28-G S T-H i s p 27-K^{-H}{ }^{-H}$, and the pETGST-Hisp27-KID ${ }^{\text {loop }}$ plasmids used as templates. The cassettes and the pET28flp27 vector were digested with Pstl and Sacll enzymes followed by cloning the cassettes into the digested vector.

\subsection{Preparation of retroviral shuttle vectors}

The MSCV-I-GFP vector was the starting plasmid. A vector map of the MSCV-I-GFP vector is shown on Figure A.5. Cassettes were amplified from the

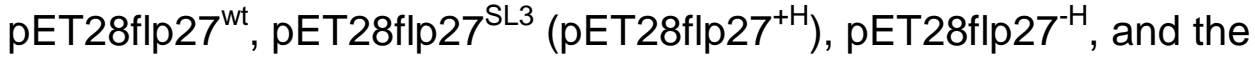
pET28flp27 $7^{\text {loop }}$ plasmids prepared in Section 2.4 above using the following primers: 5' GGACCCGAATTCATGTCAAACGTGCGAGTGTCTAACGGGAGCCCTAG 3' and 5'CAGACCGAATTCTTATTACGCATAATCCGGCACATCATACGGATACGTTTGACGTCTTCTGAGGCCAGGCTTCTTGGGC 3’. The primers were designed such that the amplified cassettes had EcoRI sites at both the 5' and 3' ends. The cassettes were digested with EcoRI and ligated into the MSCV-I-GFP vector. The plasmids were purified using an endonuclease free plasmid kit (Qiagen, Valencia, CA) and the sequences of the plasmid constructs were confirmed by DNA sequencing. The sequences of the MSCV-I-GFPflp27 $7^{\text {wt }}$, MSCV-I-GFPflp27 ${ }^{\text {SL3 }}\left(\right.$ MSCV-I-GFPflp27 ${ }^{+H}$ ), MSCV-I-GFPflp27 ${ }^{-H}$, and the MSCV-I-GFPfIp27 $7^{\text {loop }}$ are shown on Table A.6. 


\subsection{Expression and purification of Cip/Kip linker domain peptides}

The linker domain peptides were expressed as fusion proteins with aminoterminal glutathione S-transferase and histidine tags (GST-His). The fusion proteins were expressed in expressed in E. coli BL21 (DE3) cells (Novagen, San Diego, CA) at $37^{\circ} \mathrm{C}$ in LB media and induced with $1 \mathrm{mM}$ Isopropyl $\beta$-D-1thiogalactopyranoside (IPTG) when the culture reached mid-log phase (absorbance at $\left.600 \mathrm{~nm}\left(\mathrm{~A}_{600}\right) \approx 0.8\right)$. Cells were allowed to grow for 2 hours following induction. Cells were harvested by centrifugation at 5,000 $\mathrm{g} g$ for 10 minutes at $4{ }^{\circ} \mathrm{C}$ using a Sorvall Evolution RC centrifuge (Mandel Scientific, Ontario, Canada). The harvested cells were then resuspended in a nickel chelating column loading buffer (Ni-A buffer; 20 mM Tris pH 8.0, $500 \mathrm{mM} \mathrm{NaCl}, 5$ $\mathrm{mM}$ imidazole). Every gram of cell pellet was resuspended in $10 \mathrm{ml}$ of $\mathrm{Ni}-\mathrm{A}$ buffer. The resuspended cells were lysed by sonication using a Branson sonifier 450 (Branson, Danbury, CT). The cell lysate was cleared of cell debris by centrifugation at $20,000 \times \mathrm{g}$ for 30 minutes at $4{ }^{\circ} \mathrm{C}$ followed by filtration using a $0.45 \mu \mathrm{m}$ Millex syringe filter (Millipore, Bedford, MA). Unless stated otherwise, this procedure was used prepare the soluble extract after protein over-expression in E. coli. The fusion proteins were then purified from the soluble cell extract using $\mathrm{Ni}^{2+}$-affinity chromatography using a Hi-Trap nickel chelating column (GE Healthcare, Piscataway, NJ). With the exception of the resin used in the high performance liquid chromatography columns, all chromatography resins used for protein purification were obtained from GE Healthcare, Piscataway, NJ. The soluble extract was loaded onto the nickel chelating column followed by a two 
step washing procedure. In the first step, the column was washed with five column volumes of Ni-A buffer. This was followed by a second wash step in which the column was washed with five column volumes of a buffer containing $90 \% \mathrm{Ni}-\mathrm{A}$ buffer and $10 \%$ of nickel column elution buffer (Ni-B; $20 \mathrm{mM}$ Tris $\mathrm{pH}$ 8.0, $500 \mathrm{mM} \mathrm{NaCl}, 500 \mathrm{mM}$ imidazole). The fusion protein was eluted from the column by washing with a buffer containing $30 \% \mathrm{Ni}-\mathrm{A}$ and $70 \% \mathrm{Ni}-\mathrm{B}$. The eluted protein fraction was dialyzed against GST-A buffer $(140 \mathrm{mM} \mathrm{NaCl}, 2.7 \mathrm{mM} \mathrm{KCl}$, $10 \mathrm{mM} \mathrm{Na}_{2} \mathrm{HPO}_{4}, 1.8 \mathrm{mM} \mathrm{KH}_{2} \mathrm{PO}_{4}, 5 \mathrm{mM}$ dithiothreitol, $\mathrm{pH}$ 7.3) overnight at $4{ }^{\circ} \mathrm{C}$. This was followed by glutathione-affinity chromatography. The protein was loaded onto a glutathione affinity column, washed with five column volumes of GST-A buffer and eluted from the column by washing with five column volumes of GST elution buffer (GST-B buffer; $50 \mathrm{mM}$ Tris $\mathrm{pH}$ 8.0, $10 \mathrm{mM}$ reduced glutathione, $5 \mathrm{mM}$ dithiothreitol). The fraction eluted from the glutathione affinity column was dialyzed against the high performance liquid chromatography sample buffer (Buffer D; $10 \mathrm{mM}$ Tris pH 7.2, $5 \mathrm{mM}$ dithiothreitol). This was followed by thrombin cleavage at $4^{\circ} \mathrm{C}$. Thrombin (No vagen, San Diego, CA) was added to the cleavage reaction at a concentration of 0.5 units per milligram of fusion protein. The cleaved protein was then purified using high performance liquid chromatography (HPLC). The cleaved protein was mixed in a 1:1 ratio (volume/volume) with the HPLC loading buffer (HPLC-A; 0.1\% trifluoroacetic acid in water) and loaded onto a C18 column (Vydac, Hesperia, CA). The protein was eluted from the column by increasing the concentration of the HPLC elution buffer (HPLC-B; $0.1 \%$ trifluoroacetic acid in acetonitrile) at a rate of $1 \%$ per 
minute up to a concentration of $80 \%$ HPLC-B. The HPLC silica-based column used here requires use of buffers with salt concentrations lower than $150 \mathrm{mM}$ and $\mathrm{pH}$ lower than 7.5. Higher salt and $\mathrm{pH}$ conditions are detrimental to the performance and longevity of the column. The fractions were then lypholized and analyzed by mass spectrometry. Figure 2.6 shows the purification of the p27 linker domain peptide. Similar results were achieved for the p21 and p57 linker domain peptides. The mass spectrometry analyses of the linker domain peptides are shown on Figure 2.7 and Figure 2.8.

\subsection{Expression and purification of p27-KID variants}

The p27-KID linker domain mutants listed in Table A.5 were expressed in E.coli as described in Section 2.6. Like the linker domain peptides, the bacterial cells that contained the expressed protein were resuspended in $\mathrm{Ni}-\mathrm{A}$ buffer. After cell lysis and clearing, some of the mutants were found to be insoluble. Table A.6 shows all of the p27-KID mutants expressed, their solubilities, and the corresponding expression plasmid(s). Only the mutants with soluble extracts were purified. The soluble extract was loaded onto a Hi-Trap nickel column, followed by washing using five column volumes of Ni-A buffer. Protein was eluted from the column by washing with $100 \% \mathrm{Ni}$-B buffer. The eluted protein was dialyzed overnight against thrombin cleavage buffer (Buffer T; $20 \mathrm{mM}$ Tris $\mathrm{pH}$ 8.0, $50 \mathrm{mM} \mathrm{NaCl}, 5 \mathrm{mM}$ dithiothreitol) at $4{ }^{\circ} \mathrm{C}$. The cleaved protein was boiled for 20 minutes and centrifuged at $30,000 \mathrm{~g}$ for 30 minutes at $4{ }^{\circ} \mathrm{C}$. The pellet mainly contains heat-denatured $E$. coli proteins and the supernatant 

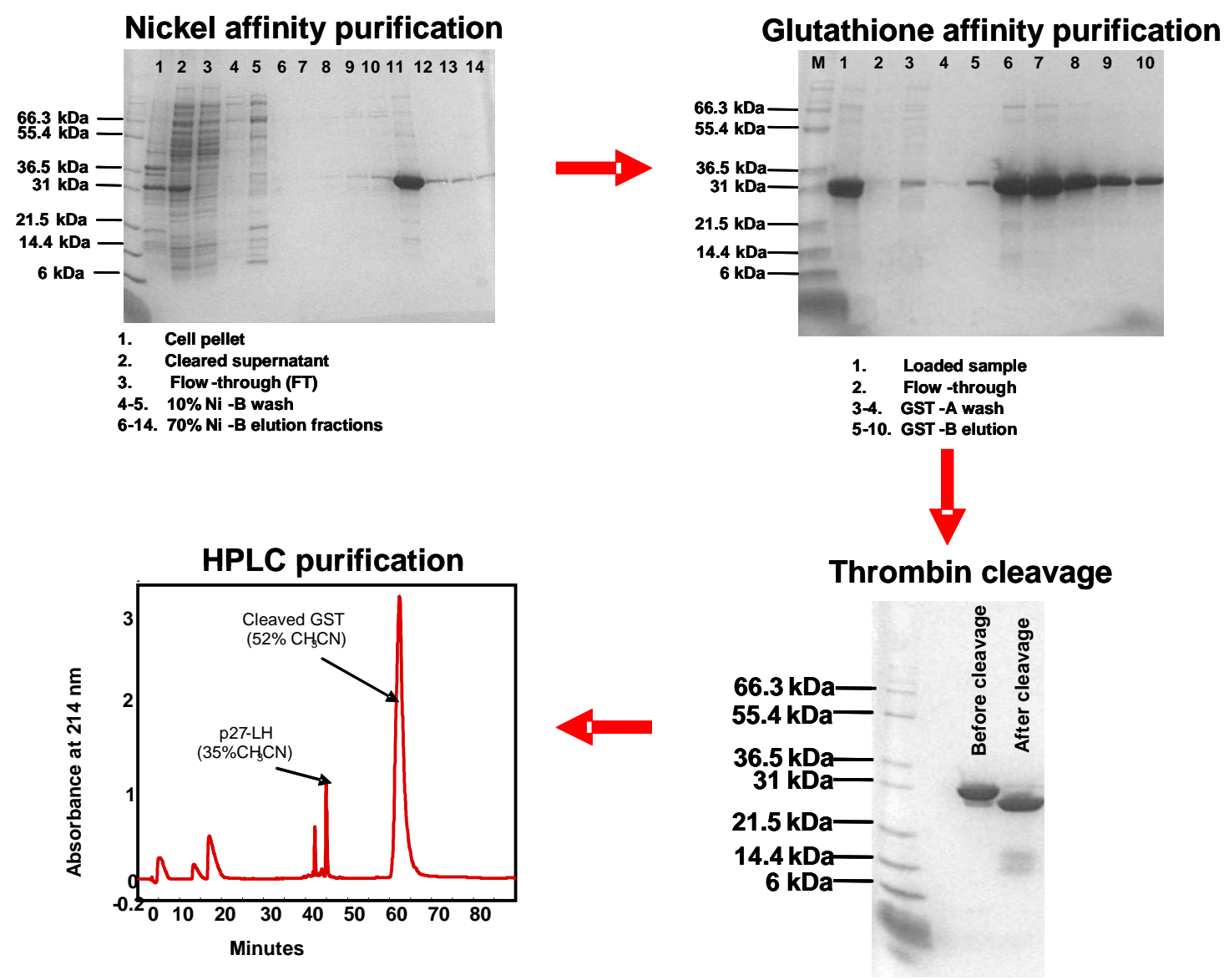

Figure 2.6 Purification of the p27 linker domain peptide (p27-LH). 

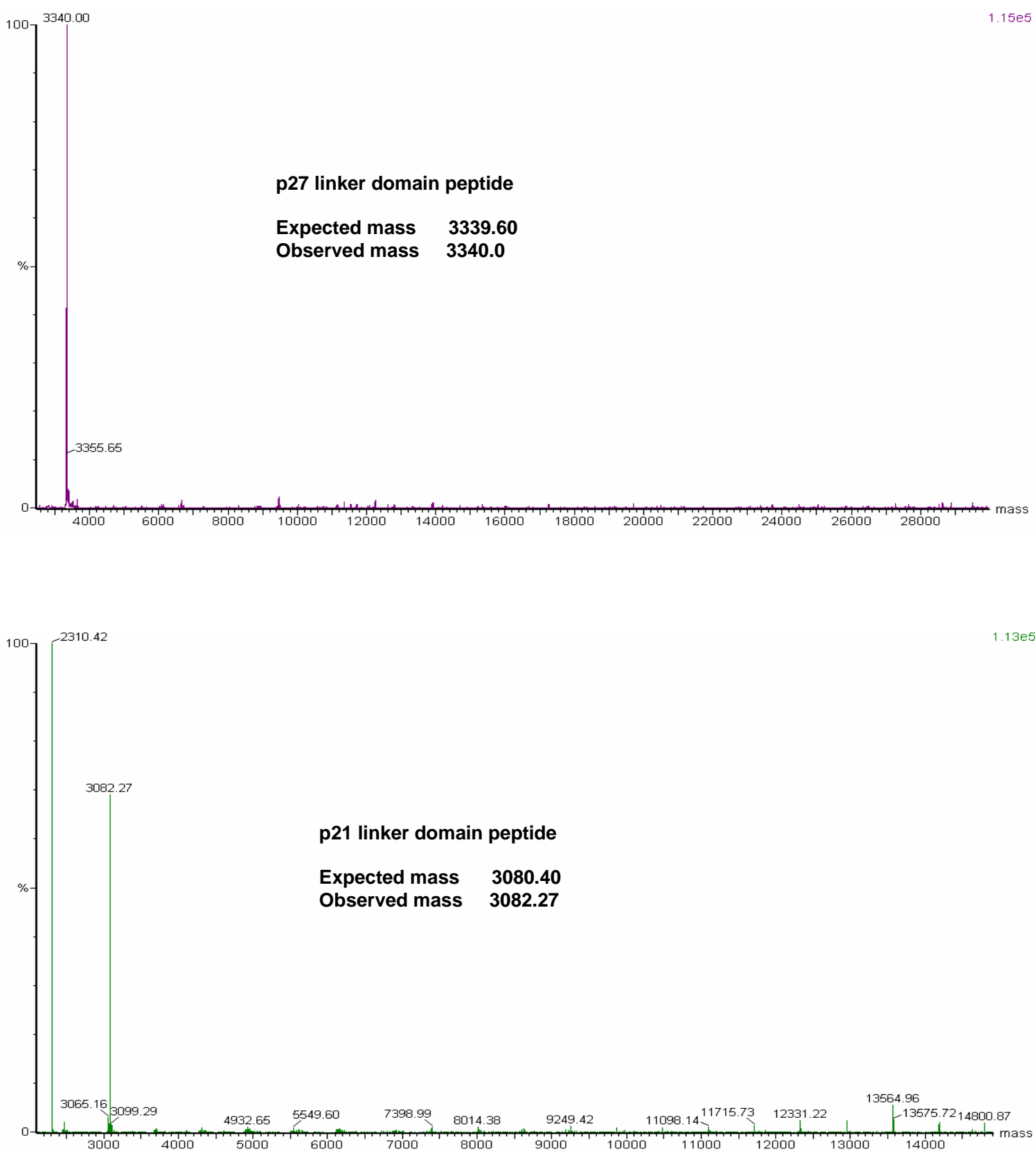

Figure 2.7 Mass spectrometry analyses of p27 and p21 linker domain peptides. 


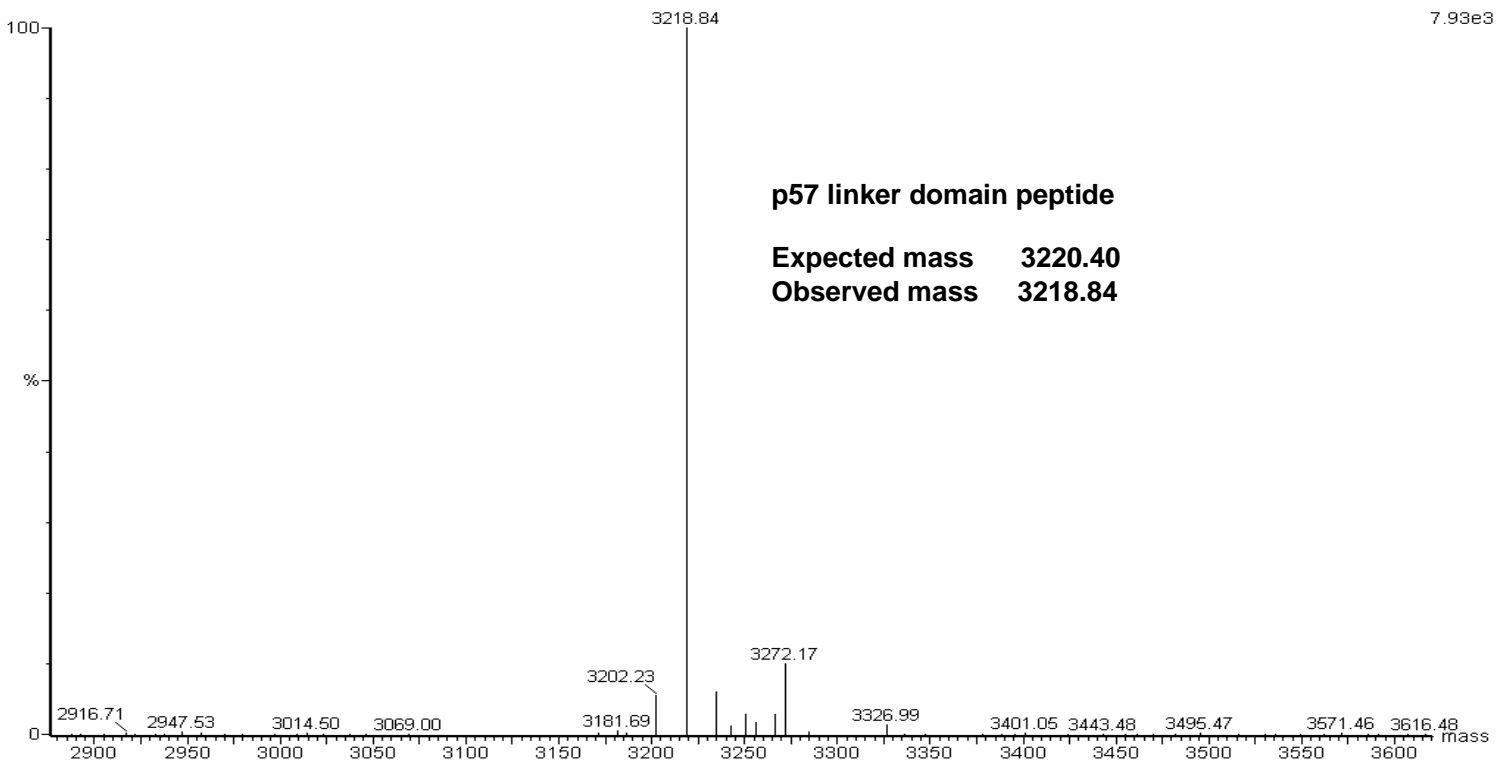

Figure 2.8 Mass spectrometry analysis of p57 linker domain peptide. 
contains the over-expressed mutant p27 protein. Figure 2.9 shows the purification of the $\mathrm{p} 27-\mathrm{KID}^{-\mathrm{H}}$ mutant. Similar results were achieved in the expression of the other p27-KID linker domain mutants. After purification, the secondary structures of the more and less helical p27-KID mutants were determined by circular dichroism spectropolarimetry. For the more helical mutant the $\mathrm{p} 27-\mathrm{KID}^{\mathrm{S} L 3}$ was found to exhibit the desired secondary structural properties and was chosen for further study. Hereafter, this mutant is referred to as the p27-KID ${ }^{+H}$. The p27-KID ${ }^{-H}$ and p27-KID loop mutants were also found to have the desired structural properties. The p27-KID linker domain mutants that I studied in detail include: p27-KID ${ }^{+H}, p 27-K^{-H}{ }^{-H}, p 27-K^{10 o p}, p 27-K^{1 D^{p 21 h e l i x ~}}$, p27$\mathrm{KID}^{\mathrm{p} 57 \text { helix }}$, and $\mathrm{p} 27-\mathrm{KID}^{\Delta \mathrm{C}}$. These mutants were characterized by mass spectrometry (Figure 2.10, Figure 2.11, and Figure 2.12) and sodium dodecyl sulfate polyacrylamide gel electrophoresis (SDS-PAGE). Figure 2.13 shows the SDS-PAGE gels of the purified proteins.

The D1 peptide (residues 23-57) was prepared by solid phase peptide synthesis (Hartwell Center, St. Jude Children's Research Hospital, Memphis, TN). A pET28a plasmid encoding a peptide corresponding to the Cdk binding domain of p27 (D2; residues 58-105) was used to express a His-tagged D2 fusion protein. The D2 peptide was purified using the same protocol as used for the KID variants described above (the nickel buffers used in the purification of D2 were phosphate based, D2-Ni-A and D2-Ni-B, as specified in Section A.1). After purification, the D2 peptide was analyzed by mass spectrometry (Figure 2.14). Prior to isothermal titration calorimetry (ITC) experiments, preparation of 


\section{Nickel affinity purification}

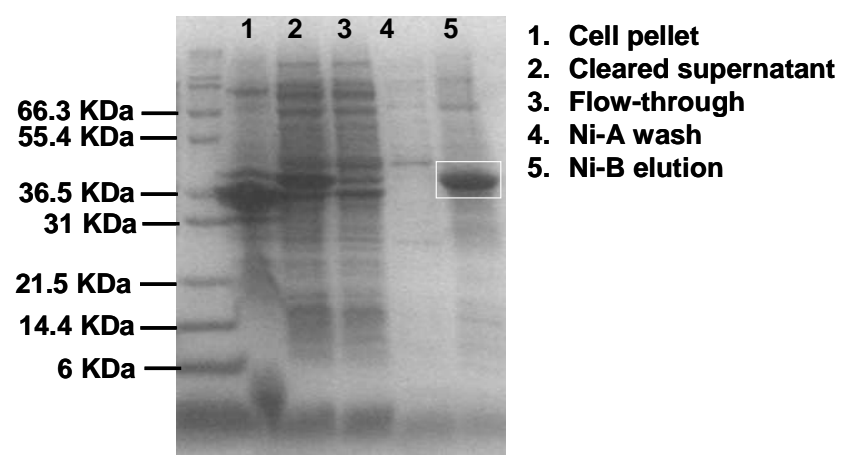

\section{Thrombin cleavage}

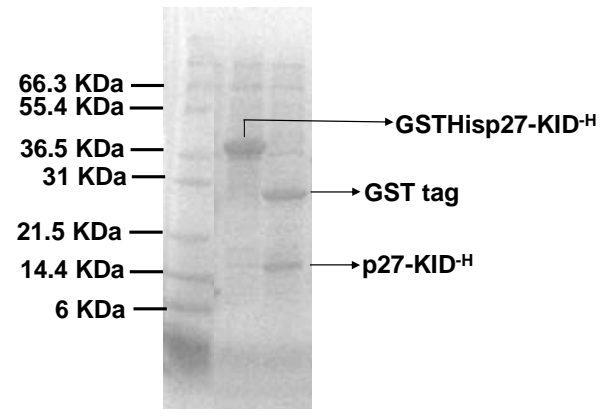

3. Boiling

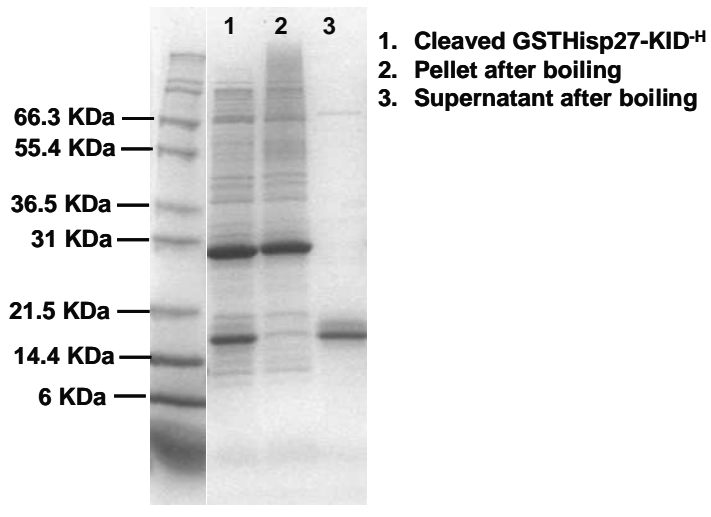

Figure 2.9 Purification of the p27-KID ${ }^{-H}$ mutant. 

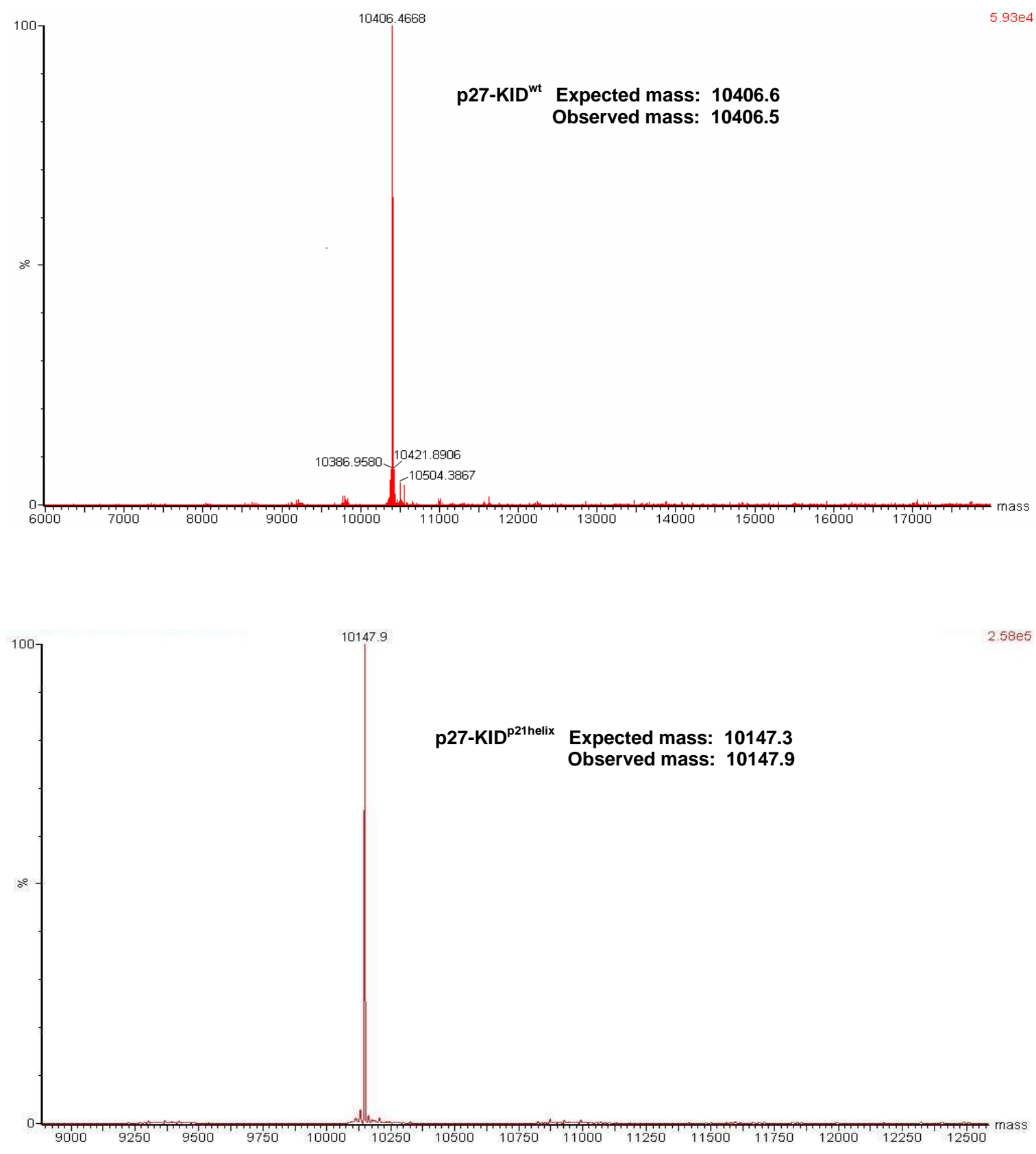

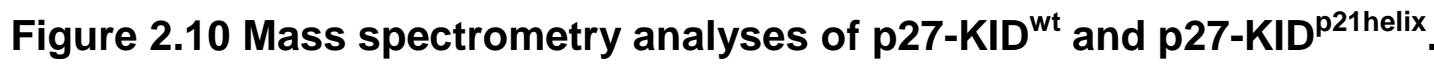



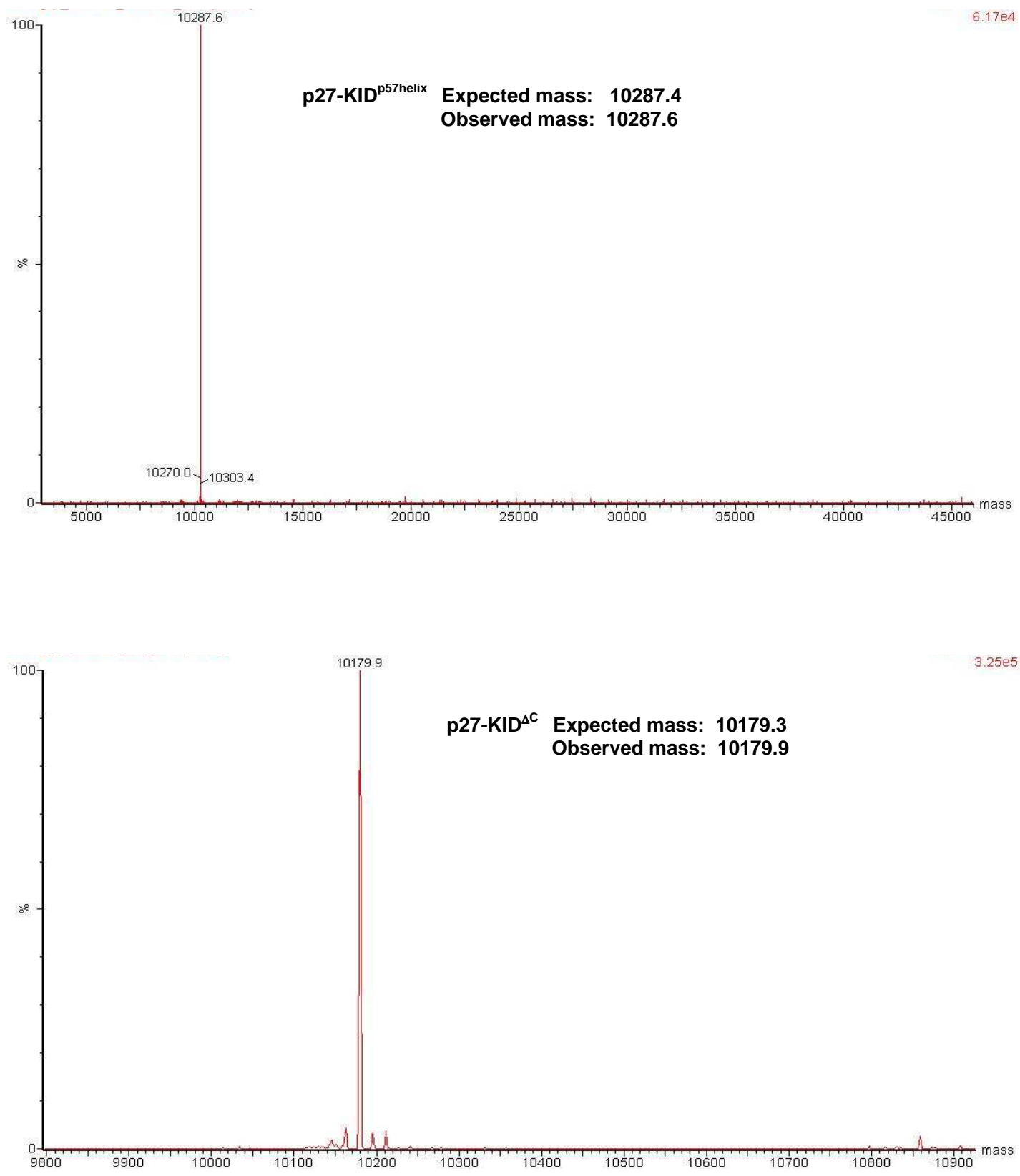

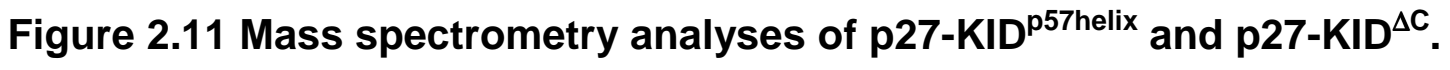



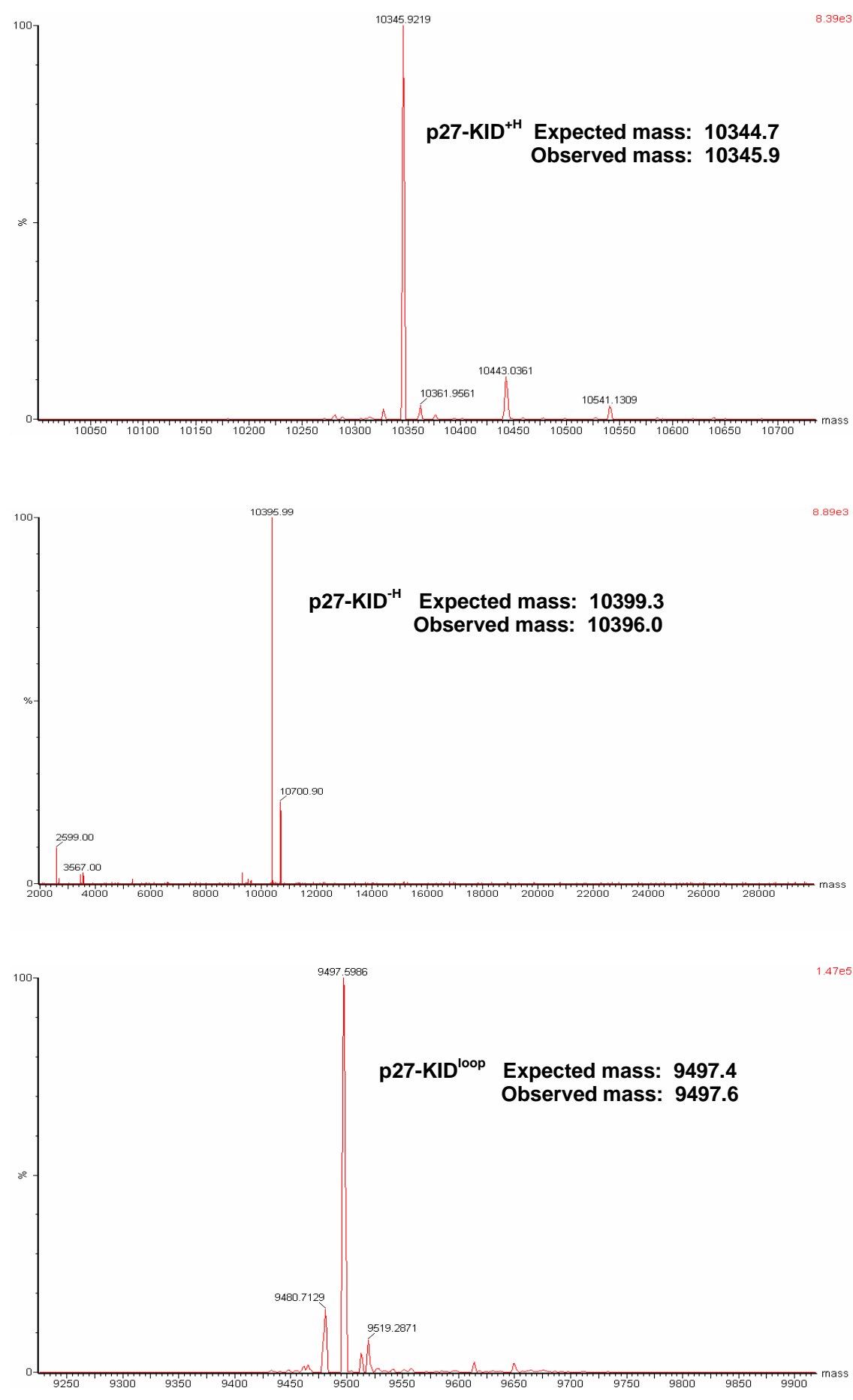

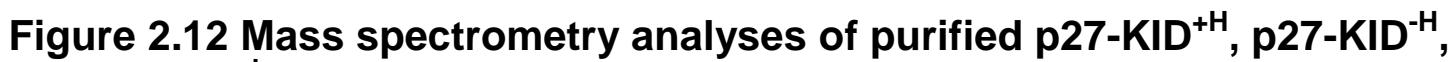
and P27-KID ${ }^{\text {loop }}$. 

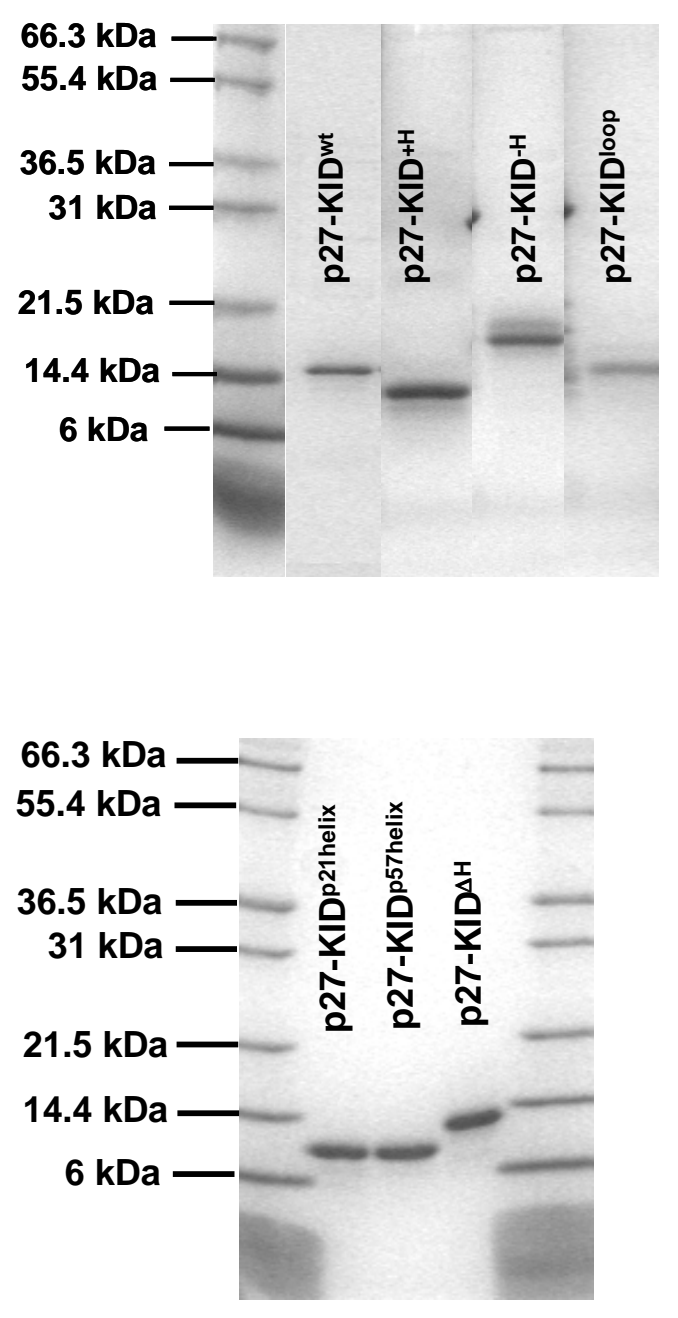

Figure 2.13 SDS-PAGE analysis of purified p27-KID mutants. Note that the lanes in the upper panel were digitally captured from multiple, individual SDSPAGE gels. However, each of these gels was electrophoresed for a similar period of time and, thus, this composite image accurately represents the relative mobility of the molecular weight markers and the p27-KID proteins. 


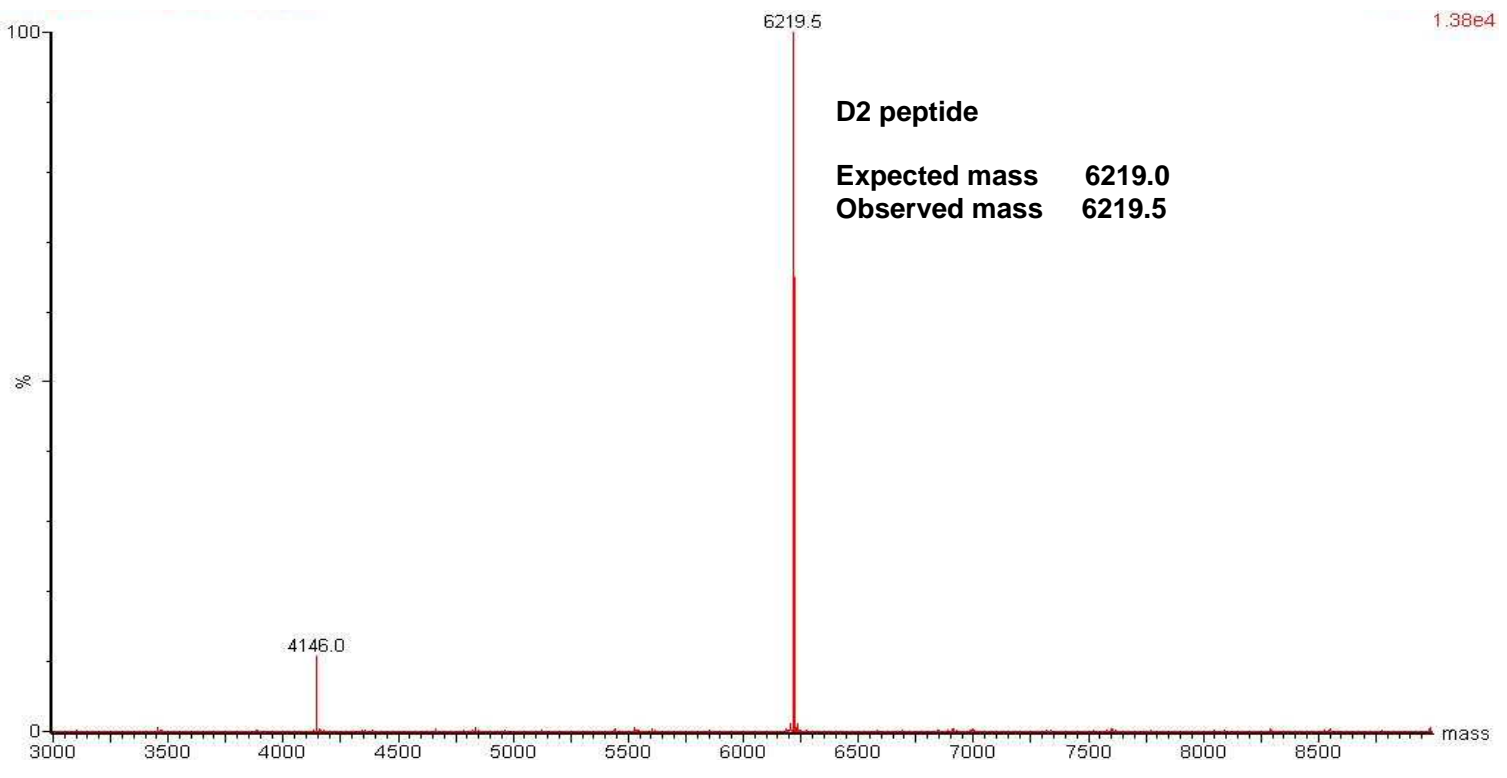

Figure 2.14 Mass spectrometry analysis of the D2 peptide. 
ternary complexes with Cdk2/cyclin A for thermal denaturation experiments or kinase assays, p27-KID ${ }^{\mathrm{wt}}$ and its variants were treated with $100 \mathrm{mM}$ dithiothreitol (DTT) at room temperature for 30 minutes followed by gel filtration chromatography purification using an Superdex S-75 column equilibrated with buffer GF (20 mM HEPES pH 7.5, $300 \mathrm{mM} \mathrm{NaCl,} 5 \mathrm{mM} \mathrm{DTT}$ ). Unless stated otherwise, all gel fitration chromatography purifications were performed in columns equilibrated with buffer GF. The D2 peptide was also treated in the same manner as the p27-KID mutants before ITC experiments and kinase assays.

\subsection{Expression and purification of cyclin A and phosphorylated Cdk2}

A pET28 plasmid encoding human cyclin A (residues 173-432) had previously been prepared in the lab. ${ }^{197}$ Cyclin A was expressed in E.coli at $37^{\circ} \mathrm{C}$ in LB media grown to early log phase $\left(A_{600} \approx 0.4\right)$. The temperature was reduced to $20^{\circ} \mathrm{C}$ and protein expression was induced with $1 \mathrm{mM}$ IPTG when the cell density reached an $A_{600} \approx 0.8$. The cells were then cultured for 20 hours and harvested by centrifugation. The protein was purified by nickel affinity chromatography (as described in Section 2.7; 50\% Ni-B was used for elution) followed by ammonium sulfate $\left(\left(\mathrm{NH}_{4}\right)_{2} \mathrm{SO}_{4}\right)$ precipitation. Maximal precipitation of the cyclin A protein during the $\left(\mathrm{NH}_{4}\right)_{2} \mathrm{SO}_{4}$ precipitation were achieved by adding finely ground $\left(\mathrm{NH}_{4}\right)_{2} \mathrm{SO}_{4}\left(0.56 \mathrm{~g}\right.$ of $\left(\mathrm{NH}_{4}\right)_{2} \mathrm{SO}_{4}$ for every $\mathrm{ml}$ of protein solution; $85 \%$ saturation) to protein solutions with a total protein concentration

greater than $3 \mathrm{mg} / \mathrm{ml}$. The precipitated protein was resuspended in TcycA -buffer 
(20 mM Tris $\mathrm{pH} 8.0,1.0 \mathrm{M} \mathrm{NaCl}, 2.5 \mathrm{mM} \mathrm{CaCl}_{2}$ ) and cleaved using thrombin overnight at $4{ }^{\circ}$. The cleaved cyclin A protein wa s further purified by anion exchange chromatography using $\mathrm{Q}$-sepharose resin and stored at $-20^{\circ} \mathrm{C}$ after a second $\left(\mathrm{NH}_{4}\right)_{2} \mathrm{SO}_{4}$ precipitation step. Before use in experiments, the stored protein was purified by gel filtration chromatography using a superdex S-75 column.

A bicistronic plasmid vector encoding full length human Cdk2 and civ1 (a yeast CAK $)^{216}$ was kindly provided by Dr. Jane Endicott, Oxford University, Oxford, UK. Both the Cdk2 and civ1 proteins are fused to GST tags at their $\mathrm{N}$-termini. civ 1 is a Cdk-activating kinase that phosphorylates Cdk2 on threonine-160. ${ }^{217}$ This vector was used to express P-Cdk2, full length human Cdk2 phosphorylated at threonine-160. P-Cdk2 expression was performed using the same procedure as discussed earlier for truncated human cyclin A. The protein was first purified by glutathione-affinity chromatography followed by dialysis into Pre-Scission cleavage buffer (50 mM Tris pH 7.0, $150 \mathrm{mM} \mathrm{NaCl}, 1$ mM EDTA, 1 mM DTT). The purified protein was cleaved overnight using Pre-Scission protease (GE Healthcare, Piscataway, $\mathrm{NJ}$ ) enzyme at $4^{\circ} \mathrm{C}$. Only the GST-P-Cdk2 fusion protein contains the Pre-Scission cleavage site and ipso facto only the P-Cdk2 protein is cleaved from the GST fusion protein. Cleaved PCdk2 was purified using a second glutathione-affinity chromatography step. GST-civ1, the cleaved GST tag from GST-P-Cdk2 fusion protein, uncleaved GST-P-Cdk2, and pre-scission enzyme (Pre-Scission is GST tagged) were retained on the column while cleaved P-Cdk2 flowed through the column. 
P-Cdk2 was then concentrated by ammonium sulfate precipitation (in the same way as cyclin A above) and stored at $-20^{\circ} \mathrm{C}$. Befor e use in experiments, the stored P-Cdk2 protein was purified by gel filtration chromatography superdex S-75 column.

To prepare the P-Cdk2/cyclin A binary complex, the ammonium sulfate precipitated P-Cdk2 and cyclin A stored at $-20^{\circ} \mathrm{C}$ were first separately dissolved in buffer GF and the concentration of each protein was determined. The two proteins were then mixed in a 1:1 mole ratio (purified cyclin A to purified P-Cdk2) and incubated at $4{ }^{\circ} \mathrm{C}$ for 30 minutes. The binary complex was then purified by gel filtration chromatography using a Superdex 75 (S-75) column. The p27-KID/P-Cdk2/cyclin A ternary complexes used in the thermal denaturation experiments were made by mixing $\mathrm{p} 27-\mathrm{KID}^{\mathrm{wt}}$ or mutants in a 1.1:1.0 mole ratio with the P-Cdk2/cyclin A complex, incubating the mixture for 30 minutes at $4{ }^{\circ} \mathrm{C}$ and purifying the complex using gel filtration chromatography using a Superdex 200 (S-200) column equilibrated in the thermal denaturation buffer (1 mM phosphate $\mathrm{pH}$ 7.0, $25 \mathrm{mM} \mathrm{NaCl}, 1 \mathrm{mM} \mathrm{DTT})$.

\subsection{Preparation of retrovirus}

Retroviruses integrate their genetic material into the genomes of their hosts and use the transcription and translation machinery of the host to express viral proteins. ${ }^{218}$ The viral genetic material is replicated by the host cell and new viruses are packaged. The packaged viruses exit the cell and invade the neighboring cells. The viral genome has four DNA modules that are 
indispensable in invasion and replication: (i) a packaging signal $(\Psi)$ the enables packaging of the viral genome into viral particles; (ii) the env gene that encodes the viral coat proteins used in the attachment of a virus to a host; (iii) the pol gene that encodes the viral polymerase; and (iv) the gag gene that encodes proteins that form the viral core structure. ${ }^{219,220}$ Viral particles are only packaged when the $\Psi$ packaging signal is in cis with the the gag, pol and env genes. ${ }^{221}$ Retroviral expression systems were designed to take advantage of the modularity of the viral genome..$^{222-224}$ First, a gene of interest (in this case flp27 $7^{\mathrm{wt}}$ or its variants) in cis with the retroviral packaging signal is ligated into shuttle vector. The gag, pol, and env genes were ligated into one plasmid or two separate plasmids called helper plasmid(s). The helper plasmid(s) and the shuttle vector are then transfected into a packaging cell line. ${ }^{225}$ The packaging cell lines have very high transfection efficiencies and are engineered to optimally and stably express the viral gag, pol, and env genes thereby ensuring production of high viral titres. Further, the packaging cell lines are genetically modified to prevent production of replication-competent viruses. ${ }^{226,227}$ In the packaging cell line, the gag, pol and env genes cloned into the helper plasmid(s) facilitate the packaging of the gene of interest into viral particles in trans. Only the gene of interest is packaged since it is in cis with the $\Psi$. The viral particles with the gene of interest are then used to transduce a non-packaging cell line. Since there are no gag, pol or env genes in the non-packaging cell line, the viruses with the gene of interest can only transduce the cells but cannot replicate. For my studies, the MSCV-I-GFP shuttle vectors in which the flp2 $7^{\mathrm{wt}}$ or its variants were cloned 
(described in Section 2.5) and a helper plasmid were used to prepare retroviruses.

First, the the viruses were packaged in the 293T packaging cell line.100 $\mathrm{mm} \times 10 \mathrm{~mm}$ plates of $293 \mathrm{~T}$ cells were cultured at $37^{\circ} \mathrm{C}$ in Dulbecco's Modified Eagle Medium (DMEM) supplemented with 10\% fetal bovine serum (FBS), $2 \%$ of $200 \mathrm{mM}$ glutamine (vol:vol), and $1 \%$ of $10,000 \mu \mathrm{g} / \mu \mathrm{l}$ penicillin/streptomycin (vol:vol). Hereafter, the DMEM supplements are referred to as additives. $3 \times 10^{6}$ cells were cultured on each plate. After 24 hours, the cell culture media (with additives) was substituted with additive free media (DMEM only) and incubated for 3 hours. During this time, transfection cocktails were prepared.

The cocktails contained a single helper DNA (containing the viral gag-polenv genes), shuttle vector DNA plasmid, fugene 6 transfection reagent (Roche, Basel, Switzerland) and additive free DMEM media. The helper DNA was obtained from Dr. Martine Roussel (St. Jude Children's Research Hospital, Memphis, TN). In every cocktail, the ratio of the volume of the fugene reagent to the amount of the shuttle vector (by mass) was varied to identify the ratio that yielded the highest viral titre. The volume of the fugene reagent was kept constant at $15 \mu \mathrm{l}$ and the amount of the shuttle vector added was varied to give different fugene to shuttle vector ratios. Cocktails with volume of fugene to mass of shuttle plasmid DNA ratios of 1:20,1:15, 1:12, and 1:10 were prepared for each construct (each construct had 4 cocktails). A constant $6 \mu \mathrm{g}$ of helper DNA was added to each coctktail. Serum free media was added to bring the total volume of each cocktail to $700 \mu$ l. Before addition of fugene to the cocktails, it 
was first warmed to room temperature according to manufacturer's instructions. For each cocktail, the reagents were added in the following order: (i) serum free media, (ii) helper DNA, (iii) vector DNA, and (iv) fugene. The cocktails were then mixed. After 3 hours, the transfection cocktails were added to the incubated cells.

After 20 hours the additive free media was replaced with $10 \mathrm{ml}$ of fresh media supplemented with additives. In the following steps, all the media was supplemented with additives unless stated otherwise. After 6 hours, the media was aspirated from the plates and $5 \mathrm{ml}$ of media was added to each plate. After 18 hours, $5 \mathrm{ml}$ of the media containing virus was harvested and $5 \mathrm{ml}$ of fresh media was added to each plate. 8 hours later, $5 \mathrm{ml}$ of media containing virus was again harvested and a fresh $7 \mathrm{ml}$ of fresh media added to each plate. Virus-containing media $(7 \mathrm{ml})$ was harvested a final time after an additional 20 hours. The harvested virus was filtered using a $0.22 \mu \mathrm{m}$ steriflip filter (Millipore, Millerica, MA) and stored at $4^{\circ} \mathrm{C}$.

\subsection{Viral transduction and cell cycle arrest analysis}

$3 \times 10^{5} \mathrm{NIH} 3$ T3 cells were cultured on $100 \times 10 \mathrm{~mm}$ plates in DMEM media with additives (additives specified in section 2.9 above). After 20 hours, the media was aspirated from each plate. $5 \mathrm{ml}$ of cold virus (virus kept in ice) supplemented with $30 \mu \mathrm{l}$ of $1 \mathrm{mg} / \mathrm{ml}$ of polybrene $(6 \mu \mathrm{l}$ of polybrene for every $\mathrm{ml}$ of virus) was added to each plate and incubated at $37^{\circ} \mathrm{C}$. After 3 hours, $10 \mathrm{ml}$ of DMEM media with additives was added to each plate. After 48 hours, the cells 
were washed, re-suspended in additive-free DMEM media, and the viral titre determined using GFP fluorescence analysis (Flow Cytometry Facility, St. Jude Children's Research Hospital, Memphis, TN). Before GFP analysis, viable cells were separated from non-viable cells by propidium iodide $(\mathrm{PI})$ staining. For $\mathrm{PI}$ staining, the cells were first mixed until a single suspension of cells was realized. The cells were then counted and washed once with phosphate buffered saline (PBS). The supernatant was decanted and the cells were resuspended in PI solution $(0.05 \mathrm{mg} / \mathrm{ml}$ propidium iodide $)$ at $1 \times 10^{6} \mathrm{cells} / \mathrm{ml}$. This was followed by treatment with RNAse for 30 minutes at room temperature. Prior to and after the RNAse step, the cells were kept in ice. The cells were then filtered using a 40 $\mu \mathrm{m}$-diameter mesh (Small Parts Inc., Miami Lakes, FL) to eliminate large multicellular clumps that might clog the flow cytometer. The filtered cells were analyzed by flow cytometry.

For cell cycle analysis, viral solutions with high viral titres, as determined by GFP analysis, were used to transduce NIH $3 \mathrm{~T} 3$ cells as described above. The cells were washed, and analyzed for viral transduction using GFP analysis. The top $10 \%$ GFP positive cells were stained by PI and analyzed for DNA content. Each cell cycle arrest analysis was performed in triplicate for each construct.

\subsection{Protein concentration determination}

The concentrations of the prepared proteins were determined by UV absorbance at $280 \mathrm{~nm}$ in a denaturing buffer containing $20 \mathrm{mM}$ phosphate $\mathrm{pH}$

6.5, and 6.0 M guanidinium hydrochloride. ${ }^{228,229}$ The molar extinction coefficients 
were determined using the ProteinParameters tool (ExPASy server;

http://us.expasy.org/tools/protparam.html) as: $15220 \mathrm{M}^{-1} \mathrm{~cm}^{-1}$ for p27-KID

p27-KID ${ }^{\text {p57helix }}, p 27-K{ }^{\Delta C}, p 27-K^{2} D^{+H}, p 27-K^{-H}$ and p27-KID ${ }^{\text {loop }} ; 15470$

$\mathrm{M}^{-1} \mathrm{~cm}^{-1}$ for D2 and p27-KID ${ }^{\mathrm{p} 21 \text { helix; }}$ and $35560 \mathrm{M}^{-1} \mathrm{~cm}^{-1}, 31860 \mathrm{M}^{-1} \mathrm{~cm}^{-1}$ and

$33710 \mathrm{M}^{-1} \mathrm{~cm}^{-1}$ for P-Cdk2, cyclin A, and the P-Cdk2/cyclin A complex respectively. The extinction co-efficient for the p27-KID ${ }^{\mathrm{wt}}, \mathrm{p} 27-\mathrm{KID}^{\mathrm{p} 57 \text { helix, }}$ p27-KID ${ }^{\Delta \mathrm{C}}, \mathrm{p} 27-\mathrm{KID}^{+\mathrm{H}}, \mathrm{p} 27-\mathrm{KID}^{-\mathrm{H}}$ and $\mathrm{p} 27-\mathrm{KID}^{\text {loop }}$ ternary complexes was determined as $24465 \mathrm{M}^{-1} \mathrm{~cm}^{-1}$. An extinction coefficient value of $24590 \mathrm{M}^{-1} \mathrm{~cm}^{-1}$ was used for the p27-KID ${ }^{\text {p21helix }}$ ternary complex. The p27, p21, and p57 linker domain peptides all had an extinction coefficient of $5690 \mathrm{M}^{-1} \mathrm{~cm}^{-1}$.

\subsection{Secondary structure analysis by circular dichroism (CD) spectropolarimetry}

The secondary structures of the p27-KID and its mutants were analyzed using an AVIV model 62A DS CD spectropolarimeter. All the samples were analyzed in a buffer containing $10 \mathrm{mM}$ phosphate $\mathrm{pH} 7.0$ and $5 \mathrm{mM}$ DTT. The experiments were performed at $25^{\circ} \mathrm{C}$. At least $3 \mathrm{CD}$ traces were averaged for each sample with a 5 second signal averaging time for each data point. To determine the helical contents of the p27-KID mutants relative to the wild-type, the CD spectra of all the proteins to be compared were obtained under exactly the same conditions. For these experiments, the protein concentration in each sample was $20 \mu \mathrm{m}$. The CD spectra were collected in a $1 \mathrm{~mm}$ path length $C D$ cuvette to maximize the signal to noise ratio. 


\subsection{Thermal denaturation experiments}

The thermal denaturation of the ternary complexes formed by the $\mathrm{p} 27-\mathrm{KID}^{\mathrm{wt}}$ and its mutants on binding the P-Cdk2/cyclin A complex was monitored in the temperature range of $15^{\circ} \mathrm{C}$ to $95^{\circ} \mathrm{C}$ using an AVIV model $62 \mathrm{~A}$ DS circular dichroism spectropolarimeter equipped with a Pelltier thermoelectric temperature control device. The samples, each containing $400 \mathrm{nM}$ of protein in $1 \mathrm{mM}$ sodium phosphate buffer, $\mathrm{pH} 7.0,25 \mathrm{mM} \mathrm{NaCl}$ and $1 \mathrm{mM}$ DTT, were analyzed in a $1 \mathrm{~cm}$ path length quartz cuvette. For these studies, denaturation was monitored by measuring ellipticity at $222 \mathrm{~nm}$ at $1{ }^{\circ} \mathrm{C}$ intervals at a heating rate of $5{ }^{\circ} \mathrm{C} \min ^{-1}$ with constant stirring. Three experiments were performed with ternary complexes comprised of P-Cdk2/cyclin A and p27-KID ${ }^{w t}, p 27-K D^{+H}, p 27-K D^{-H}$, p27-KID ${ }^{\text {loop }}$, or $\mathrm{p} 27-\mathrm{KID}^{\mathrm{p} 57 \text { helix }}$, and two experiments were performed with ternary complexes containing $\mathrm{p} 27-\mathrm{KID}^{\mathrm{p} 21 \text { helix }}$ or $\mathrm{p} 27-\mathrm{KID}^{\Delta \mathrm{C}}$. The denaturation curves were analyzed as previously described by Bowman, et al., ${ }^{216}$ as follows: The fraction of unfolded protein $\left(f_{\mathrm{D}}\right)$ at any given temperature was calculated from the corresponding experimental ellipticity value $\theta$ using Equation 2.1:

$$
f_{\mathrm{D}}=\left[\theta-\left(\theta_{\mathrm{N}}+m_{\mathrm{N}} T\right)\right] /\left[\left(\theta_{\mathrm{D}}+m_{\mathrm{D}} T\right)-\left(\theta_{\mathrm{N}}+m_{\mathrm{N}} T\right)\right]
$$

where $T$ is the temperature, $\theta_{\mathrm{N}}$ and $\theta_{\mathrm{D}}$ are the ellipticity values corresponding to the native $(\mathrm{N})$ and denatured $(\mathrm{D})$ states extrapolated to $T=0$, and $m_{\mathrm{N}}$ and $m_{\mathrm{D}}$ are the slopes of the baselines preceding and following the transition region obtained 
by linear regression analysis. The free energy of unfolding, $\Delta G_{D-N}$, was calculated using Equation 2.2:

$$
\Delta G_{\mathrm{D}-\mathrm{N}}=-R T \ln K=-\mathrm{RT} \ln \left\{f_{\mathrm{D}} /\left(1-f_{\mathrm{D}}\right)\right\}
$$

to calculate $T_{\mathrm{m}}, \Delta G_{\mathrm{D}-\mathrm{N}}$ values over the transition region of the denaturation curve were plotted against temperature and fit to a straight line by linear regression. $T_{\mathrm{m}}$ corresponds to the intercept of the $\mathrm{x}$-axis where $\Delta G_{\mathrm{D}-\mathrm{N}}$ is equal to zero (this is the temperature at which $f_{\mathrm{D}}=f_{\mathrm{N}}$ ). The melting temperature for each sample was determined at least two times. The experimental error in the determination of the melting temperature was calculated by determining the standard deviation from the mean $T_{\mathrm{m}}$ for each sample.

\subsection{Determination of thermodynamic parameters by ITC}

The thermodynamic parameters associated with $\mathrm{p} 27-\mathrm{KID}^{\mathrm{wt}}$ and the mutant proteins binding to P-Cdk2/cyclin A were determined using isothermal titration calorimetry (ITC). The experiments were performed using a VP-ITC calorimeter (Microcal, Northampton, MA). A major advantage of ITC is that the enthalpy of association $(\Delta \mathrm{H})$, binding stoichiometry $(n)$, and binding affinity $\left(K_{\mathrm{a}}\right.$; within certain limits) of the two interacting components can be determined in a single experiment. It is important to note that ITC cannot accurately determine the binding affinity associated with very tight interactions (low $\mathrm{nM}$ binding affinity) because such systems have a very high $c$ value $-c$ is defined by Wiseman, et 
al. ${ }^{230}$ However, accurate estimates can be obtained. The Gibbs free energy of association $(\Delta G)$ is calculated using the equation $\Delta G=-R T \ln \left(1 / K_{a}\right)$, where $R$ is the gas constant $\left(1.987 \mathrm{cal} \mathrm{mol}^{-1} \mathrm{~K}^{-1}\right)$ and $\mathrm{T}$ is the temperature (degrees Kelvin) at which the experiment was performed. Given that the above limitation applies, the values of the Gibbs free energy of association of p27-KID ${ }^{w t}, \mathrm{p} 27-\mathrm{KID}^{+\mathrm{H}}$, p27-KID ${ }^{-H}, p 27-K D^{p 57 h e l i x}, p 27-K D^{p 21 h e l i x}$ and p27-KID ${ }^{\Delta C}$ binding to P-Cdk2/cyclin A are taken as estimates of the upper limit of the $K_{d}$ and those of p27-KID ${ }^{\text {loop }}, \mathrm{D} 1$, and D2 are considered to be accurate.

The experiments were performed at $25^{\circ} \mathrm{C}$ in buffer containing $20 \mathrm{mM}$ HEPES pH 7.5, $300 \mathrm{mM} \mathrm{NaCl}$ and $5 \mathrm{mM}$ DTT. For these experiments, the syringe volume was $250 \mu \mathrm{l}$ and the cell volume was $1.3 \mathrm{ml}$. In each experiment, the P-Cdk2/cyclin A complex was in the cell and p27-KID ${ }^{\text {wt }}$ or its variants were in the syringe (titrant). A standard injection protocol was used for each titrant. $2 \mu \mathrm{L}$ of the titrant was first injected followed by 39 injections, $6 \mu \mathrm{L}$ each, with 300 seconds between the injections. The following were the protein concentrations used in the ITC studies: $75 \mu \mathrm{M}$ p27-KID ${ }^{\mathrm{wt}}+7.4 \mu \mathrm{M}$ P-Cdk2/cyclin A; $73 \mu \mathrm{M}$ D1 + 5.2 $\mu \mathrm{M}$ P-Cdk2/cyclin A; $72.3 \mu \mathrm{M} \mathrm{D2}+5.61 \mu \mathrm{M}$ P-Cdk2/cyclin A; $56 \mu \mathrm{M}$ p27-KID ${ }^{+H}+4.2 \mu \mathrm{M}$ P-Cdk2/cyclin A; $42 \mu \mathrm{M}$ p27-KID ${ }^{-H}+4.4 \mu \mathrm{M}$ P-Cdk2/cyclin A; and 36.4 $\mu \mathrm{M}$ p27-KID loop $+3.1 \mu \mathrm{M}$ P-Cdk2/cyclin A; $56.87 \mu \mathrm{M}$ p27-KID ${ }^{\mathrm{p} 2 \text { helix }}$ + 6.22 $\mu \mathrm{M}$ P-Cdk2/cyclin A; $53.57 \mu \mathrm{M}$ p27-KID ${ }^{p 57 \text { helix }}+4.6 \mu \mathrm{M}$ P-Cdk2/cyclin A; and $35 \mu \mathrm{M}$ p27-KID ${ }^{\Delta C}+2.88 \mu \mathrm{M}$ P-Cdk2/cyclin A. The samples were degassed before the experiments were performed and the instrument was regularly calibrated as specified by the manufacturer. The binding data were fit to a $1: 1$ 
binding model using the Origin software (Origin Lab Corporation, Northampton, MA). This analysis yielded values of the enthalpy of binding $(\Delta \mathrm{H})$, the equilibrium association constant $\left(K_{\mathrm{a}}\right)$, and the Gibbs free energy of binding $(\Delta \mathrm{G})$. The product of entropy and temperature (-T $\Delta S$ ) was calculated using Equation 2.3:

$$
-T \Delta S=\Delta G-\Delta H
$$

\subsection{Kinase inhibition assays}

A solution of $80 \mathrm{pM}$ P-Cdk2/cyclin A binary complex containing $2.5 \mu \mathrm{M}$ histone $\mathrm{H} 1$ (Upstate, Charlottsville, VA) and varying amounts of $\mathrm{p} 27-\mathrm{KID}^{\mathrm{wt}}$ or its mutants was incubated at $4{ }^{\circ} \mathrm{C}$ for 1 hour. $6 \mu \mathrm{Ci}$ of $\mathrm{Y}^{32} \mathrm{P}$-ATP (PerkinElmer, Inc., Wellesley, MA) was added to each reaction and incubated at $30^{\circ} \mathrm{C}$ for 35 minutes. The concentration of the $\mathrm{Y}^{32} \mathrm{P}$-ATP was $1 \mathrm{mCi} / \mathrm{ml}(1$ part $10 \mathrm{mCi} / \mathrm{ml}$ of $\mathrm{Y}^{32} \mathrm{P}$-ATP to 9 parts $111 \mu \mathrm{M}$ cold ATP) and the total reaction volume was $15 \mu \mathrm{l}$. All the samples were in a kinase assay buffer containing $20 \mathrm{mM} \mathrm{HEPES} \mathrm{pH,} \mathrm{7.3,}$ $25 \mathrm{mM}$ sodium b-glycerolphosphate, $15 \mathrm{mM} \mathrm{MgCl}_{2}, 16 \mathrm{mM}$ EGTA, $0.5 \mathrm{mM}$ $\mathrm{Na}_{3} \mathrm{VO}_{4}$ and $10 \mathrm{mM}$ DTT. The kinase reactions were quenched by adding SDS-PAGE gel loading buffer. The samples were then analyzed by SDS-PAGE and the bands quantified using a phosphoimager (Molecular Dynamics, Sunnyvale, CA). The inhibition data was fit to a dose-response model using Graphpad Prism software (Graphpad Software, Inc., San Diego, CA). Each curve was fit from a triplicate set of experiments. The error was reported as the $95 \%$ confidence interval of the $\mathrm{IC}_{50}$ from a curve fit of a triplicate data set. 


\section{Chapter 3: The linker domains of proteins in the Cip/Kip family play similar structural roles}

The structure of the linker domain of p21, p27, and p57 is conserved, and this domain serves to couple the D1 and D2 domains of Cip/Kip proteins. In the first study of this chapter, I have investigated the effects of deleting the linker domain on the function of p27 as a cyclin-dependent kinase inhibitor. The thermodynamics of association of peptides corresponding to the D1 and D2 domains of the KID with the P-Cdk2/cyclin A complex relative to the p27-KID ${ }^{\text {wt }}$ were analyzed. The functional consequences of deleting the linker domain were determined by studying inhibition of the P-Cdk2/cyclin A catalytic activity by D2 peptide.

Although the nascent helical structure of the linker domain is conserved amongst members of the Cip/Kip family, its sequence is not. However, the sequence of the linker domain for each Cip/Kip protein is highly conserved in different species suggesting that the sequences of these proteins may be important for their function. In the second study in this chapter, I investigated whether the linker domains of the Cip/Kip proteins, despite having different sequences, play similar structural roles. Chimeric Cip/Kip proteins were used for these studies. The chimeric proteins were prepared by replacing the linker domain of the p27-KID with either the p21 linker domain (p27-KID ${ }^{\mathrm{p} 21 \text { helix }}$ ) or the p57 linker domain (p27-KID $\left.{ }^{p 57 h e l i x}\right)$. The interaction of these chimeric proteins with the P-Cdk2/cyclin A kinase complex was then probed. This chimeric mutant 
model is suitable for these studies for two reasons. First, the individual linker domains of p21, p27 and p57 have an inherent propensity to form $\alpha$-helices indicating that this domain can fold independent of the rest of the KID polypeptide. Secondly, the residues neighboring the linker domains in the native proteins are well conserved. This minimizes the variation in stabilization of the linker helix by the neighboring residues. By replacing only the linker domain and keeping D1 and D2 constant, the consequences of linker domain swapping on the interaction with the P-Cdk2/cyclin A complex can be studied.

Only 3 out of the 22 residues in the linker domains of p21, p27, and p57 are conserved. The role of these conserved residues in the interaction of the Cip/Kip proteins with Cdk complexes is unknown. In the final study of this chapter, I have investigated the role of these conserved residues by preparing a p27-KID variant in which the three conserved residues in the linker domain are mutated to glycines (Glu39Gly/Leu41Gly/Arg43Gly, the p27-KID ${ }^{\Delta C}$ mutant) and probing its interaction with the P-Cdk2/cyclin A complex.

In all the studies, CD was used to study the secondary structures of the mutants. The relative stabilities of the ternary complexes formed by the variants on binding to P-Cdk2/cyclin A were determined by monitoring the ellipticity of each complex at $222 \mathrm{~nm}$ as a function of temperature. ITC was used to determine the thermodynamics of association of the mutants with the P-Cdk2/cyclin A complex. Kinase inhibition assays were performed to provide insights into the consequences of the mutations on the kinase inhibition activity of the Cip/Kip proteins. 


\subsection{The nascent helical character of the linker domain is preserved in the chimeric mutants and the $\mathrm{p} 27-\mathrm{KID}^{\Delta \mathrm{C}}$ mutant}

The secondary structures of the p21, p27, and p57 linker domain peptides,

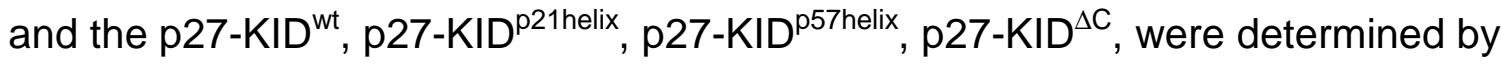
CD (Figure 3.1 and Figure 3.2). The linker domain peptides are mainly unstructured (ellipticity at about $200 \mathrm{~nm}$ ), with some helical secondary structure (ellipticity at $222 \mathrm{~nm}$ ). These results are consistent with secondary structure prediction for the linker domains of p27, p21, and p57. ${ }^{197}$ The p21 helix peptide shows a significantly higher content of random coil than either p27 or p57 linker helix peptides. This is not surprising since its sequence has a glycine (Gly40) which would effectively decrease its helical content and increase its overall random coil composition relative to the p27 and p57 helix peptides. The presence of nascent $\alpha$-helical secondary structure in all the linker domain peptides indicates that their sequences have an intrinsic tendency to form $\alpha$-helices and can fold independent of the rest of the KID sequence. The stabilization of the linker domains in the context of the KID should be very similar since the residues neighboring the linker domains in the p21, p57, and p27 proteins are well conserved.

The chimeric mutants have secondary structures very similar to that of the p27-KID ${ }^{\text {wt }}$. They are largely unstructured with nascent helical characteristics. The mean residue molar ellipticity values of the chimeric mutants at $222 \mathrm{~nm}$ $\left(\left[\Theta_{222}\right]\right)$ are comparable to that of the $\mathrm{p} 27-\mathrm{KID}^{\mathrm{wt}}$ indicating that they have comparable helical contents. The results of the secondary structural analysis of 


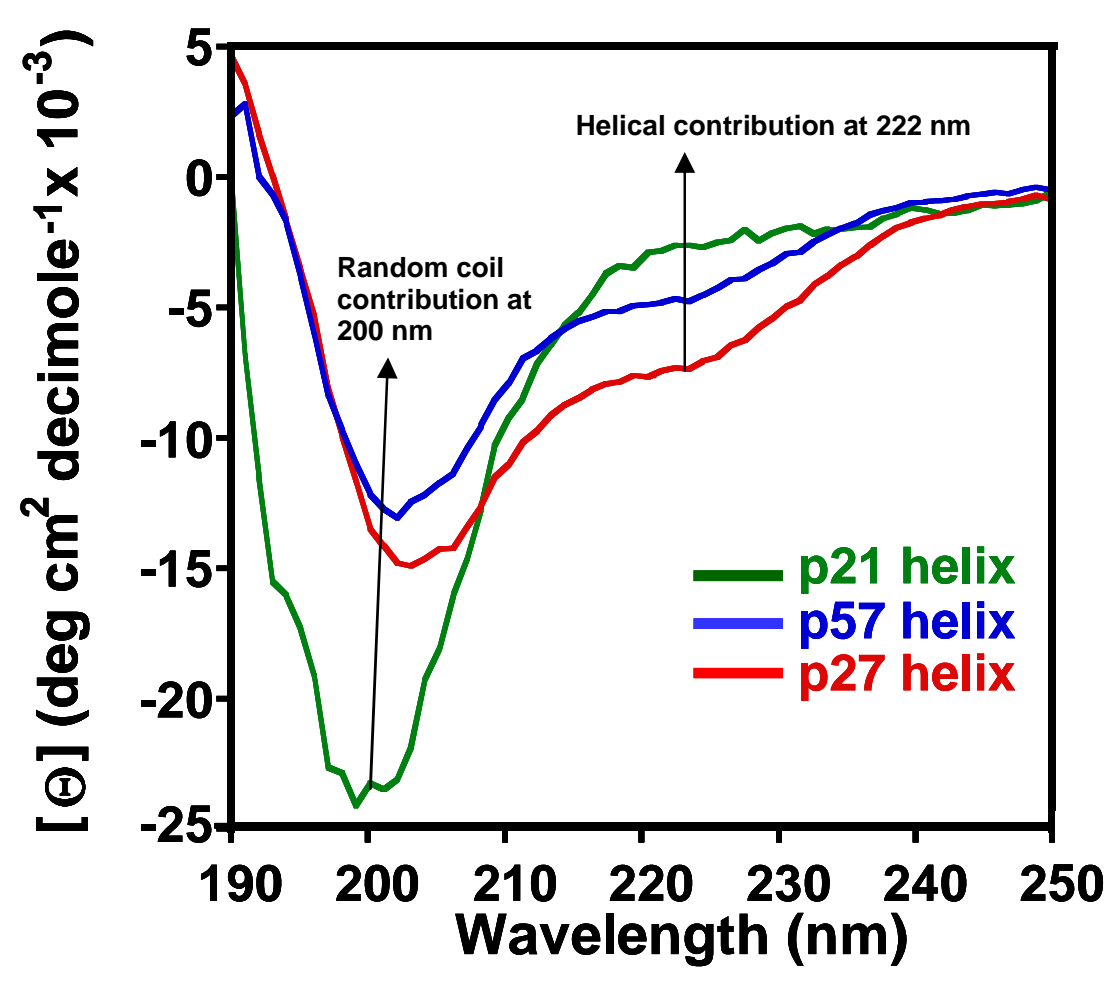

Figure 3.1 CD spectra of the p21, p27, and p57 linker domain peptides. 


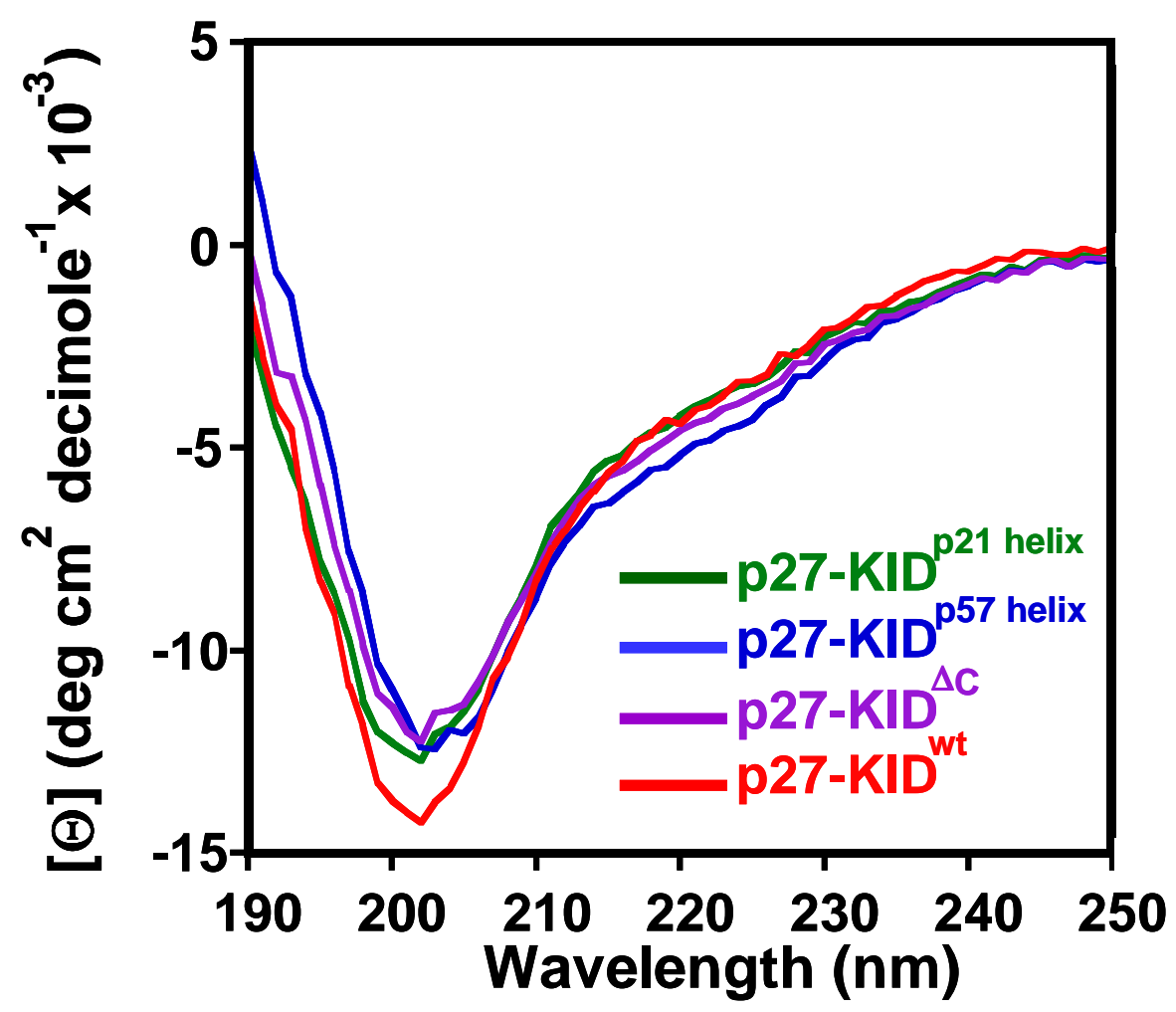

Figure 3.2 CD spectra of p27-KID ${ }^{\mathrm{wt}}, \mathrm{p} 27-\mathrm{KID}^{\mathrm{p} 21 \mathrm{helix}}, \mathrm{p} 27-\mathrm{KID}^{\mathrm{p} 57 \mathrm{helix}}$, and p27$\mathrm{KID}^{\Delta \mathrm{C}}$. 
the chimeric mutants suggest that the linker domains of p21 and p57, despite having different sequences and being bordered by D1 and D2 domains of p27, adopt structures similar to their previously reported secondary structures in the context of p21-KID and p57-KID, respectively. ${ }^{188,198}$ Further, this shows that our chimeric mutant model does not cause major changes in the secondary structure and hence is suitable for probing the structure conservation/sequence divergence relationship of the linker domains of p27, p21, and p57.

The $\mathrm{p} 27-\mathrm{KID}^{\Delta \mathrm{C}}$ mutant also exhibits a largely disordered secondary structure with nascent helical characteristics (Figure 3.2). The $\left[\Theta_{222}\right]$ of this mutant is comparable to that of the wild-type p27-KID indicating that the two p27-KID variants have comparable helical contents. These results suggest that the mutation of the conserved residues in the linker domain to glycines does not cause a major structural change. This structural similarity suggests that any disparities in the interaction between $\mathrm{p} 27-\mathrm{KID}^{\Delta \mathrm{C}}$ and the P-Cdk2/cyclin A complex relative to the $\mathrm{p} 27-\mathrm{KID}^{\mathrm{wt}}$ are not a consequence of perturbation of the structure of the linker domain.

\subsection{Binding thermodynamics determined by ITC}

The thermodynamics of the association of the $\mathrm{p} 27-\mathrm{KID}^{\mathrm{wt}}$ and its variants with the P-Cdk2/cyclin A complex were characterized by ITC. First, the thermodynamic parameters of the binding of either the D1 or the D2 peptide to the binary complex were compared to those of $\mathrm{p} 27-\mathrm{KID}^{\mathrm{wt}}$. The binding of either $\mathrm{D} 1$ or $\mathrm{D} 2$ to $\mathrm{P}-\mathrm{Cdk} 2 / \mathrm{cyclin} \mathrm{A}$ is much weaker than that of $\mathrm{p} 27-\mathrm{KID}^{\mathrm{wt}}$. The 
dissociation constants for the p27-KID ${ }^{\mathrm{wt}}$, D1, and D2 were determined to be 3.34 $\pm 1.64 \mathrm{nM}, 42.20 \pm 5.16 \mathrm{nM}$, and $82.96 \pm 17.89 \mathrm{nM}$, respectively (Table 3.1).

These results show that the coupling of D1 and D2 via the linker domain greatly increases the affinity of p27 for the kinase complex. The $\Delta G$ 's of association of p27-KID ${ }^{\mathrm{wt}}, \mathrm{D} 1$, and D2 with the P-Cdk2/cyclin A complex are $-10.06 \pm 0.07 \mathrm{kcal}$ $\mathrm{mol}^{-1}$ and $-9.66 \pm 0.12 \mathrm{kcal} \mathrm{mol}^{-1}$, and $-11.61 \pm 0.30 \mathrm{kcal} \mathrm{mol}^{-1}$, respectively. The sum of the $\Delta \mathrm{H}$ values for the interactions of $\mathrm{D} 1$ and $\mathrm{D} 2$ with $\mathrm{P}-\mathrm{Cdk2/cyclin} \mathrm{A}$ is $-47.69 \mathrm{kcal} \mathrm{mol}^{-1}$ relative to the $-43.77 \mathrm{kcal} \mathrm{mol}^{-1}$ of heat released when p27-KID ${ }^{\text {wt }}$ binds to the same complex. This suggests that the P-Cdk2/cyclin A bound conformation(s) of either the D1 or D2, or both peptides, is slightly different from its/their conformation(s) in the context of p27-KID.

The chimeric mutants bound to the P-Cdk2/cyclin A complex with affinities very similar to that of $\mathrm{p} 27-\mathrm{KID}^{\mathrm{wt}}$ (Table 3.1 ). Figure 3.3 shows the binding isotherms for the interactions between $\mathrm{p} 27-\mathrm{KID}^{\mathrm{wt}}$ or its variants with the P-Cdk2/cyclin A complex. The dissociation constants for p27-KID ${ }^{\mathrm{p} 21 \text { helix }}$ and p27-KID ${ }^{\text {p57helix }}$ were $2.83 \pm 1.37 \mathrm{nM}$, and $0.49 \pm 0.14 \mathrm{nM}$ respectively. Similar to that for p27-KID ${ }^{\mathrm{wt}}$, the binding of each chimeric mutant to the P-Cdk2/cyclin A complex was associated with a large entropic penalty. $-\mathrm{T} \Delta \mathrm{S}$ values of +32.16 $\pm 1.22 \mathrm{kcal} \mathrm{mol}^{-1},+16.24 \pm 1.29 \mathrm{kcal} \mathrm{mol}^{-1}$, and $+18.44 \pm 0.35 \mathrm{kcal} \mathrm{mol}^{-1}$ were obtained for the $\mathrm{p} 27-\mathrm{KID}^{\mathrm{wt}}, \mathrm{p} 27-\mathrm{KID}^{\mathrm{p} 21 \text { helix }}$, and $\mathrm{p} 27-\mathrm{KID}^{\mathrm{p} 57 \text { helix }}$ respectively. However, the disparities $-\mathrm{T} \Delta \mathrm{S}$ values suggest that the chimeric mutants do not fold to the same extent as the p27-KID ${ }^{w t}$. Although there are differences in the folding of the chimeric mutants relative the $\mathrm{p} 27-\mathrm{KID}^{\mathrm{wt}}$ on binding the 
Table 3.1. Thermodynamic parameters determined by ITC at $25^{\circ} \mathrm{C}$.

\begin{tabular}{|c|c|c|c|c|}
\hline Interaction & $\begin{array}{c}K_{\mathrm{d}} \\
(\mathrm{nM})\end{array}$ & $\begin{array}{c}\Delta \mathrm{G}^{\mathrm{a}} \\
\left(\mathrm{kcal} \mathrm{mol}^{-1}\right)\end{array}$ & $\begin{array}{c}\Delta \mathrm{H} \\
\left(\mathrm{kcal} \mathrm{mol}^{-1}\right)\end{array}$ & $\begin{array}{c}-\mathrm{T} \Delta \mathrm{S}^{\mathrm{b}} \\
\left(\mathrm{kcal} \mathrm{mol}^{-1}\right)\end{array}$ \\
\hline $\mathrm{D} 1+\mathrm{P}-\mathrm{Cdk} 2 /$ cyclin A & $42.20 \pm 5.16$ & $-10.06 \pm 0.07$ & $-16.14 \pm 0.20$ & $+6.08 \pm 0.27$ \\
\hline $\mathrm{D} 2+\mathrm{P}-\mathrm{Cdk} 2 /$ cyclin A & $82.96 \pm 17.89$ & $-9.66 \pm 0.12$ & $-31.55 \pm 1.66$ & $+21.89 \pm 1.53$ \\
\hline $\mathrm{p} 27-\mathrm{KID}^{\mathrm{wt}}+\mathrm{P}-\mathrm{Cdk} 2 /$ cyclin A & $3.34 \pm 1.64$ & $-11.61 \pm 0.30^{\circ}$ & $-43.77 \pm 1.38$ & $+32.16 \pm 1.22$ \\
\hline p27-KID & $2.83 \pm 1.37$ & $-11.70 \pm 0.27^{c}$ & $-27.94 \pm 1.26$ & $+16.24 \pm 1.29$ \\
\hline p27-KID & $0.49 \pm 0.14$ & $-12.71 \pm 0.17^{c}$ & $-31.15 \pm 0.51$ & $+18.44 \pm 0.35$ \\
\hline p27-KID ${ }^{\Delta C}+P-C d k 2 / c y c l i n A$ & $1.97 \pm 0.65$ & $-11.89 \pm 0.21^{\mathrm{c}}$ & $-39.65 \pm 0.13$ & $+27.76 \pm 0.17$ \\
\hline
\end{tabular}

In all the experiments above, the stoichiometry of binding was determined as one (1), with experimental error within $10 \%$. The errors reported above are the standard deviations from the mean of three independent experiments.

${ }^{a}$ Calculated with the equation $\Delta G=-R T \ln \left(1 / K_{d}\right)$.

${ }^{\mathrm{b}}$ Calculated with the equation $\Delta \mathrm{G}=\Delta \mathrm{H}-\mathrm{T} \Delta \mathrm{S}$.

${ }^{c}$ The $c$ values for these p27-KID variants are very high and the $\Delta G$ values reported here are taken as estimates of the upper limits of $K_{\mathrm{d}}$. Ideal c values for accurate determination of $K_{d}$ have a range of 1 to 500 . The $c$ values for the binding of $\mathrm{p} 27-\mathrm{KID}^{\mathrm{wt}}, \mathrm{p} 27-\mathrm{KID}^{\mathrm{p} 21 \text { helix }}, \mathrm{p} 27-\mathrm{KID}^{\mathrm{p} 57 \text { helix }}$, and the $\mathrm{p} 27-\mathrm{KID}^{\Delta \mathrm{C}}$ to P-Cdk2/cyclin A complex are 2212, 2195, 9387, and 1461, respectively. 


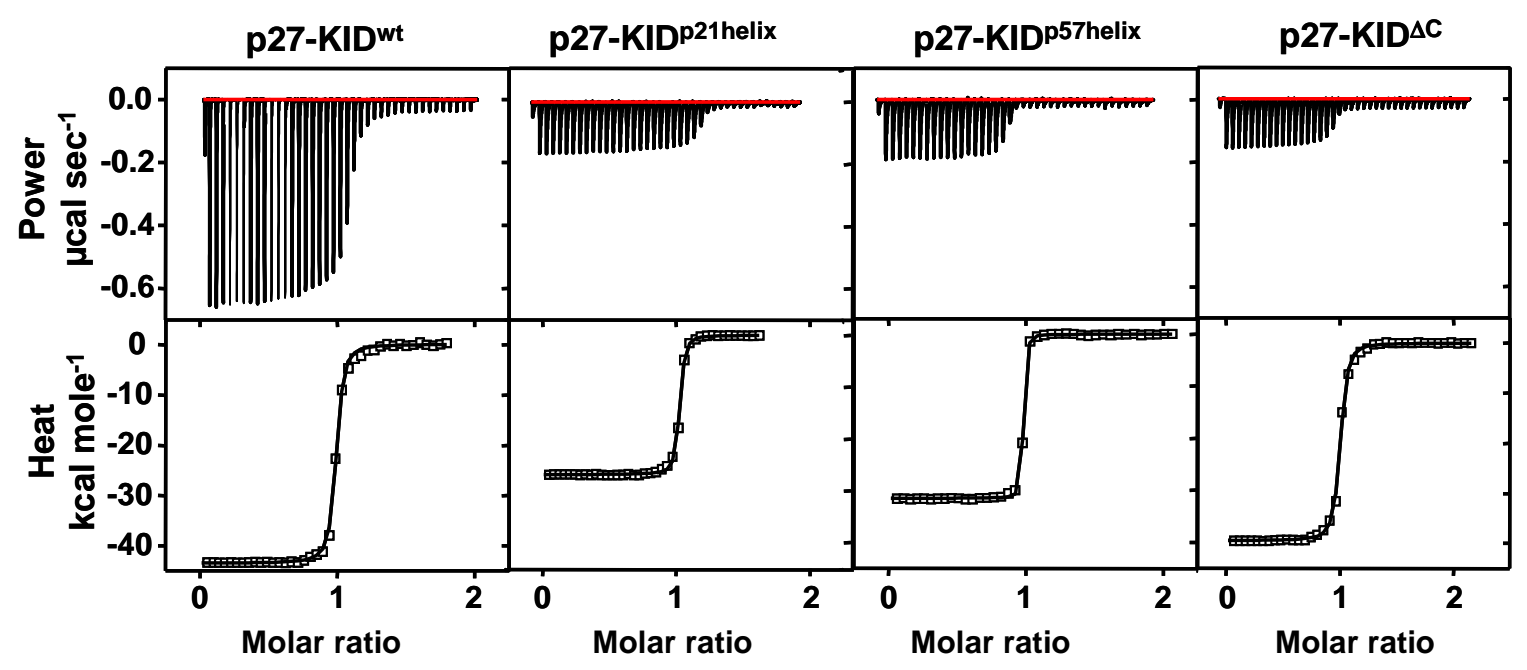

Figure 3.3 ITC binding isotherms for the p27-KID variants. 
P-Cdk2/cyclin A complex, data from the binding of D1 and D2 with the same complex suggest that the D1 and D2 domains of these mutants bind to the kinase complex. The chimeric mutants have much higher binding affinities for the P-Cdk2/cyclin A complex than either D1 or D2 (Table 3.1). This stems from the linkage by the linker domain. In addition, the association of the the chimeric mutants with P-Cdk2/cyclin A is more favorable than that of D1 or D2. Further, the Gibbs free energy of association for the chimeric mutants with P-Cdk2/cyclin A is more favorable than $\Delta G$ values previously reported for the interactions of p27-KID wt with either Cdk2 or cyclin A. ${ }^{197}$ The differences in the folding of the p27-KID ${ }^{\mathrm{wt}}$ and the chimeric mutants are further seen in the enthalpy values obtained for their interaction with the P-Cdk2/cyclin A complex. Enthalpy values of $-43.77 \pm 1.38 \mathrm{kcal} \mathrm{mol}^{-1},-27.94 \pm 1.26 \mathrm{kcal} \mathrm{mol}^{-1}$, and $-31.15 \pm 0.51 \mathrm{kcal} \mathrm{mol}^{-1}$ were obtained for the p27-KID ${ }^{w t}, \mathrm{p} 27-\mathrm{KID}^{\mathrm{p} 21 \text { helix }}$, and p27-KID ${ }^{\mathrm{p} 57 \text { helix }}$ respectively. The differences in $\Delta \mathrm{H}$ are most likely caused by the interactions of different sets of residues in the linker domains of $\mathrm{p} 27-\mathrm{KID}^{\mathrm{wt}}, \mathrm{p} 27-\mathrm{KID}^{\mathrm{p} 2 \text { helix }}$, and $\mathrm{p} 27-\mathrm{KID}^{\mathrm{p} 57 \mathrm{~h} \text { elix }}$ (recall that the sequences linker domains of the Cip/Kip proteins are not conserved). The enthalpy values show that the interactions of the chimeric mutants with the P-Cdk2/cyclin A complex have fewer favorable contacts with respect to the $\mathrm{p} 27-\mathrm{KID}^{\mathrm{wt}}$. Nevertheless, the enthalpy generated by each chimeric mutant compensates for the entropic cost associated with the folding on binding the P-Cdk2/cyclin A complex making the free energies of the interactions negative and favorable - entropy-enthalpy compensation. Entropy-enthalpy compensation is a phenomenon in which the enthalpy change in a system during 
a chemical process (in which the experimental conditions are varied) is partially or completely compensated for by a corresponding change in entropy such that the net free energy change is very small. As an example, the decrease in the absolute magnitude of $\Delta \mathrm{H}$ in the interactions of $\mathrm{p} 27-\mathrm{KID}^{\mathrm{p} 21 \text { helix }}$ and $\mathrm{p} 27-\mathrm{KID}^{\mathrm{p} 57 \mathrm{~h} \text { elix }}$ with P-Cdk2/cyclin A relative to $\mathrm{p} 27-\mathrm{KID}^{\mathrm{wt}}$ caused by the differences in the sequences of the linker domains (variation in experimental conditions) is compensated for by a proportionate decrease in the entropic cost of binding; the residues that make fewer favorable contacts (release less heat) have more degrees of freedom (increase in entropy). Entropy-enthalpy compensation therefore results in a narrow range of variation of $\Delta \mathrm{G}$ between the native system and the system with varied experimental conditions. The $\Delta G$ values of the interactions of $\mathrm{p} 27-\mathrm{KID}^{\mathrm{wt}}$, $\mathrm{p} 27-\mathrm{KID}^{\mathrm{p} 21 \text { helix }}$, and $\mathrm{p} 27-\mathrm{KID}^{\mathrm{p} 57 \text { helix }}$ with $\mathrm{P}-\mathrm{Cdk} 2 / \mathrm{cyclin} \mathrm{A}$ vary within a narrow range indicating that, despite the enthalpic differences, the linker domains have very similar $\Delta \mathrm{G}$ contributions to the interaction of the Cip/Kip proteins with the P-Cdk2/cyclin A complex. The free energies of association for the p27-KID ${ }^{\mathrm{wt}}, \mathrm{p} 27-\mathrm{KID}^{\mathrm{p} 21 \mathrm{helix}}$, and $\mathrm{p} 27-\mathrm{KID}^{\mathrm{p} 57 \mathrm{helix}}$ were $-11.61 \pm 0.30 \mathrm{kcal} \mathrm{mol}^{-1}$, $-11.70 \pm 0.27 \mathrm{kcal} \mathrm{mol}^{-1}$, and $-12.71 \pm 0.17 \mathrm{kcal} \mathrm{mol}^{-1}$ respectively.

The interaction of the p27-KID ${ }^{\Delta \mathrm{C}}$ mutant with the P-Cdk2/cyclin A complex relative to the $\mathrm{p} 27-\mathrm{KID}^{\mathrm{wt}}$ shows the thermodynamic contributions of the conserved residues within the linker domain. This mutant binds very tightly to the kinase complex with a $K_{d}$ of $1.97 \pm 0.65 \mathrm{kcal} \mathrm{mol}^{-1}$ (Figure 3.3, Table 3.1). Similar to the chimeric mutants, it also folds extensively on binding the 
$\mathrm{P}-\mathrm{Cdk} 2 /$ cyclin A complex and has a $-\mathrm{T} \Delta \mathrm{S}$ value of $+27.76 \pm 0.17 \mathrm{kcal} \mathrm{mol}^{-1}$. This extensive folding on binding, as is expected, is accompanied by the release of a large amount of heat. The enthalpy of association of the p27-KID ${ }^{\Delta C}$ mutant with $\mathrm{P}$-Cdk2/cyclin A complex is $-39.65 \pm 0.13 \mathrm{kcal} \mathrm{mol}^{-1}$. The enthalpic contribution of the conserved residues is about $4.12 \mathrm{kcal} \mathrm{mol}^{-1}$ (about $9.4 \%$ of the total heat released). The net free energy obtained for the interaction of the $\mathrm{p} 27-\mathrm{KID}^{\Delta \mathrm{C}}$ with the P-Cdk2/cyclin A complex is $-11.89 \pm 0.21 \mathrm{kcal} \mathrm{mol}^{-1}$. The $\Delta \mathrm{G}$ of the interaction of the p27-KID ${ }^{\Delta C}$ mutant to the P-Cdk2/cyclin A complex is therefore the same as that for the $\mathrm{p} 27-\mathrm{KID}^{\mathrm{wt}}$ within the error range. This shows that even though there is a slight decrease in enthalpy of interaction of the p27-KID with the P-Cdk2/cyclin A complex in the absence of the conserved residues within the linker domain, this loss is completely compensated for by a proportionately reduced entropic cost - entropy-enthalpy compensation.

\subsection{The p27-KID wt ternary complex is more stable than the corresponding complexes of its variants}

Previous studies have shown that the binding of the p27-KID to P-Cdk2/cyclin A stabilizes that binary kinase complex and increases its melting temperature $\left(T_{\mathrm{m}}\right) \cdot{ }^{216}$ While the P-Cdk2/cyclin A complex has a $T_{\mathrm{m}}$ of $54.7 \pm 0.6{ }^{\circ} \mathrm{C}$, the $\mathrm{p} 27-\mathrm{KID} / \mathrm{P}-\mathrm{Cdk} 2 /$ cyclin A ternary complex has a $T_{\mathrm{m}}$ of $82.3 \pm$ $0.5^{\circ} \mathrm{C}$, an increase of $27.6^{\circ} \mathrm{C}$ in the melting temperature. I investigated the consequences of linker domain swapping (chimeric mutants), and the role of the 
conserved residues within the linker domain on the stabilization of the binary complex by determining the $T_{\mathrm{m}}$ 's of their ternary complexes on binding the $\mathrm{P}-\mathrm{Cdk2}$ /cyclin A complex. The melting temperature $\left(T_{\mathrm{m}}\right)$ for each protein complex was determined by using standard thermal denaturation curve-fitting equations ${ }^{231}$ to analyze the temperature dependence of $\left[\Theta_{222}\right]$ using $C D$. The thermal denaturation curves for the ternary complexes of $\mathrm{p} 27-\mathrm{KID}^{\mathrm{wt}}$, p27-KID ${ }^{p 21 h e l i x}, p 27-K^{p} D^{p 57 h e l i x}$, and $p 27-K_{I} D^{\Delta C}$ are shown in Figure 3.4. It is significant to note that the thermal denaturation process for the complexes in this study is irreversible due to protein aggregation at temperatures above their $T_{\mathrm{m}}$ values. Our results show that the ternary complex containing p27-KID ${ }^{\mathrm{wt}}\left(T_{\mathrm{m}}=\right.$ $82.3 \pm 0.5^{\circ} \mathrm{C}$ ) is slightly more stable than those containing p27-KID ${ }^{\text {p21helix }}$ or p27-KID ${ }^{\text {p57helix }}$ which have $T_{\mathrm{m}}$ values of $80.7 \pm 0.2^{\circ} \mathrm{C}$ and $80.1 \pm 0.1{ }^{\circ} \mathrm{C}$ (Table 3.2), respectively. The $\mathrm{p} 27-\mathrm{KID}^{\Delta C}$ is the least stable of the three mutants relative to the p27-KID ${ }^{\mathrm{wt}}$ and has a $T_{\mathrm{m}}$ of $74.1 \pm 0.6^{\circ} \mathrm{C}$. The relative instability of the p27-KID ${ }^{\Delta C}$ is probably caused largely in part by the mutation of Leu41 which in the crystal structure of the $\mathrm{p} 27-\mathrm{KID}^{\mathrm{wt}} / \mathrm{Cdk} 2 /$ cyclin A complex ${ }^{104}$ is buried in the $\mathrm{p} 27-\mathrm{KID}^{\mathrm{wt}} / \mathrm{cyclin} \mathrm{A}$ binding interface. By mutating this residue to a glycine, I have decreased the buried surface area at this position and consequently diminished the extent of favorable inter-molecular interactions. The other conserved residues, Glu39 and Arg43, are solvent exposed and most likely do not contribute significantly to the interaction of the linker domain with the cyclin A. This result indicates that the conserved residues of the linker helix contribute significantly to the stability of the ternary complex (by about $8^{\circ} \mathrm{C}$ ). 


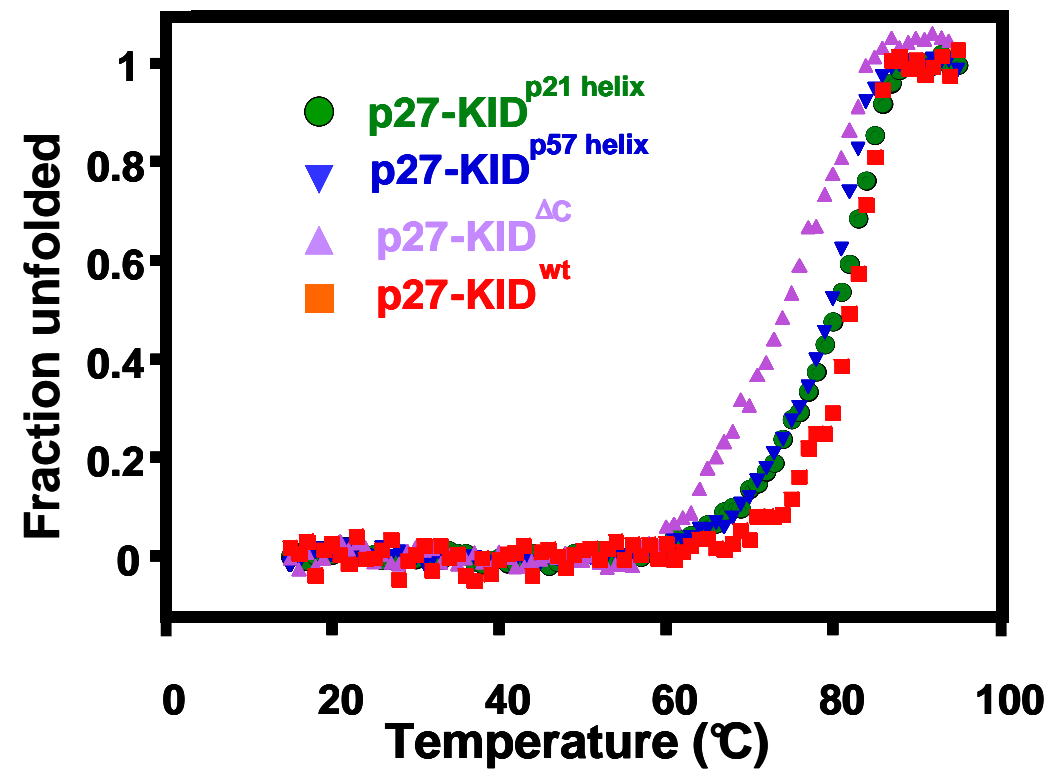

Figure 3.4 Thermal denaturation curves of the p27-KID mutants. 
Table 3.2 Melting temperatures of ternary complexes formed by either p27-KID ${ }^{\text {wt }}$ or its variants bound to P-Cdk2/cyclin A complex.

\begin{tabular}{|c|c|}
\hline Complex & $T_{\mathrm{m}}\left({ }^{\circ} \mathrm{C}\right)$ \\
\hline $\mathrm{p} 27-\mathrm{KID}^{\mathrm{wt}} / \mathrm{P}-\mathrm{Cdk} 2 /$ cyclin $\mathrm{A}^{\mathrm{a}}$ & $82.3 \pm 0.5$ \\
\hline p27-KID ${ }^{\text {p21helix } / P-C d k 2 / c y c l i n ~ A ~}$ & $80.7 \pm 0.2$ \\
\hline p27-KID & $80.1 \pm 0.1$ \\
\hline $\mathrm{p} 27-\mathrm{KID}^{\Delta \mathrm{C}} / \mathrm{P}-\mathrm{Cdk} 2 /$ cyclin $\mathrm{A}^{\mathrm{b}}$ & $74.1 \pm 0.6$ \\
\hline
\end{tabular}

\footnotetext{
${ }^{a}$ The error reported is the standard deviation from the mean of three independent experiments.

${ }^{b}$ The error reported is the standard deviation from the mean of two independent experiments.
} 


\subsection{Relative inhibition potency determined by in vitro kinase assays}

Kinase inhibiton assays were performed to probe the P-Cdk2/cyclin A kinase inhibition potency of the p27-KID mutants with respect to the p27-KID ${ }^{\mathrm{wt}}$. In these studies, the P-Cdk2/cyclin A kinase concentration was $80 \mathrm{pM}$ to avoid depletion of the inhibitors which would be the case at much higher kinase concentrations. All the proteins used in these studies were exchanged into the kinase assay buffer (buffer KA) by gel filtration chromatography. This was necessary because these assays are very sensitive to differences in solution conditions. It was therefore very important to prepare all the samples under exactly the same buffer conditions in order to obtain comparable results for the various $\mathrm{p} 27-\mathrm{KID}$ variants.

Studies of the inhibition potency of D2, Figure 3.5, Table 3.3, shows that it is a poor inhibitor $\left(\mathrm{IC}_{50}=125 \mathrm{nM}\right)$ and cannot completely inhibit kinase activity. even at very high D2 inhibitor concentrations, as high as $17.7 \mu \mathrm{M}, \mathrm{P}-\mathrm{Cdk} 2 /$ cyclin A retains about $25 \%$ of full kinase activity. This indicates that the isolated D2 peptide does not fold into the same inhibitory conformation as does D2 in the context of the full KID.

On the other hand, the chimeric mutants are potent kinase inhibitors with $I_{50}$ values in the low $\mathrm{nM}$ range. Figure 3.6 shows the gels and kinase inhibition curves of the chimeric mutants. The $\mathrm{IC}_{50}$ values for the $\mathrm{p} 27-\mathrm{KID}^{\mathrm{wt}}, \mathrm{p} 27-$

$\mathrm{KID}^{\mathrm{p} 21 \mathrm{helix}}$, and $\mathrm{p} 27-\mathrm{KID}^{\mathrm{p} 57 \text { helix }}$ were $0.22 \mathrm{nM}, 1.73 \mathrm{nM}$, and $3.45 \mathrm{nM}$ respectively (Table 3.3). These results clearly show that the D2 domains of the chimeric mutants are folded into conformations with a capacity of inhibiting the kinase 
(a)

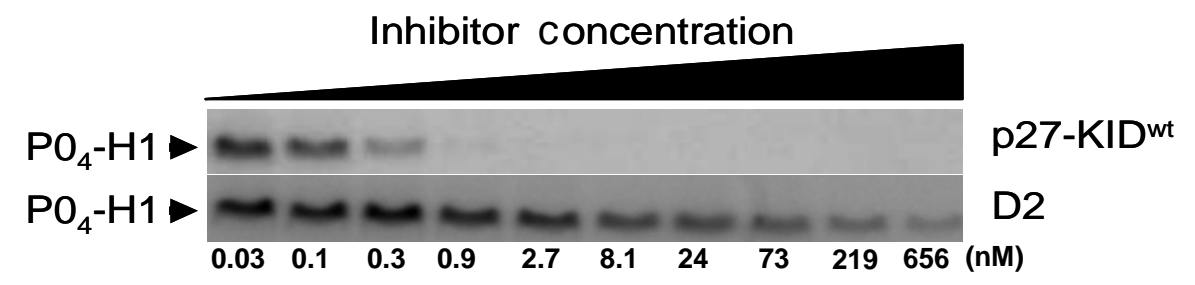

(b)

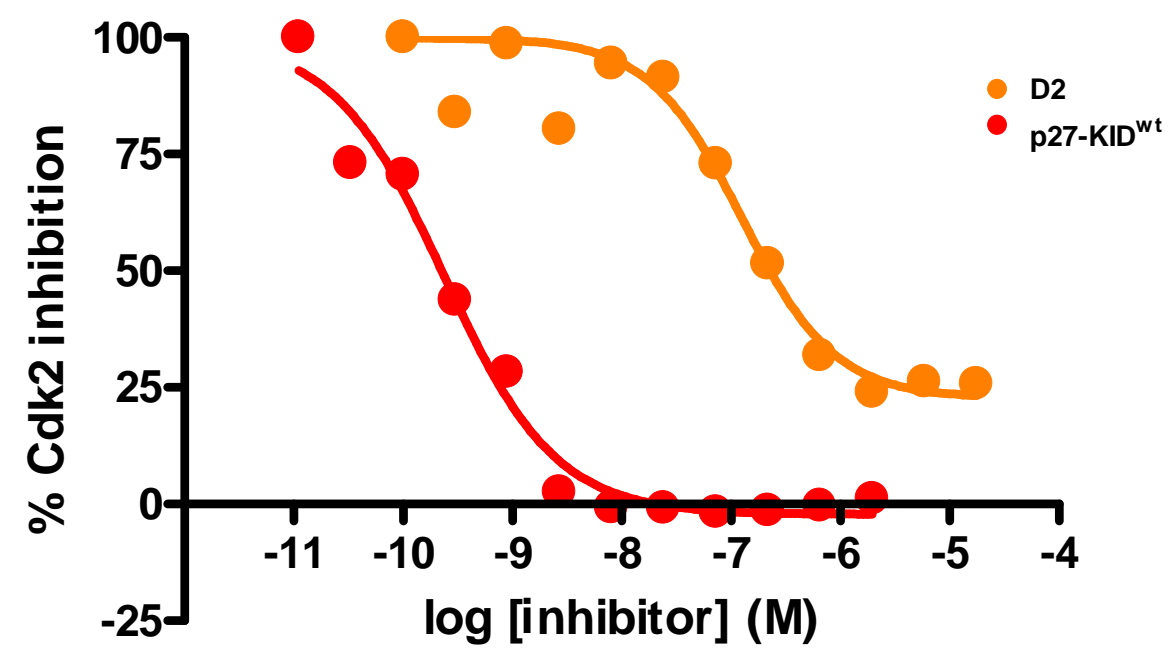

Figure 3.5 Inhibition of P-Cdk2/cyclin A kinase activity by the D2 peptide. (a) SDS-PAGE gels showing the inhibition of the P-Cdk2/cyclin A kinase by the p27-KID ${ }^{\text {wt }}$ and D2. $\mathrm{PO}_{4}-\mathrm{H} 1$ is phosphorylated histone $\mathrm{H} 1$. (b) Inhibition curves for the p27-KID ${ }^{\text {wt }}$ and $\mathrm{D} 2$ peptide. 
Table 3.3 Inhibition potencies of the p27-KID variants.

\begin{tabular}{ccc}
\hline Variant & $\mathrm{IC}_{50}(\mathrm{nM})$ & $\mathbf{9 5 \%}$ confidence intervals of $\mathrm{IC}_{50}(\mathrm{nM})^{\mathrm{a}}$ \\
\hline $\mathrm{p} 27-\mathrm{KID}^{\mathrm{wt}}$ & 0.22 & $0.17-0.28$ \\
$\mathrm{p} 27-\mathrm{KID}^{\mathrm{p} 2 \text { helix }}$ & 1.73 & $1.09-2.73$ \\
$\mathrm{p} 27-\mathrm{KID}^{\mathrm{p} 57 h \text { hlix }}$ & 3.45 & $2.32-5.13$ \\
$\mathrm{p} 27-\mathrm{KID}^{\Delta \mathrm{C}}$ & 2.56 & $1.70-3.87$ \\
$\mathrm{D} 2$ & $125^{\mathrm{b}}$ & $45.94-340.80$ \\
\hline
\end{tabular}

${ }^{a}$ Error is reported as the $95 \%$ confidence interval of the $\mathrm{IC}_{50}$ from a curve fit of a triplicate data set.

${ }^{b}$ This variant only partially inhibits kinase activity. $25 \%$ residual kinase activity observed at inhibitor concentrations as high as $17.7 \mu \mathrm{M}$. 
(a)

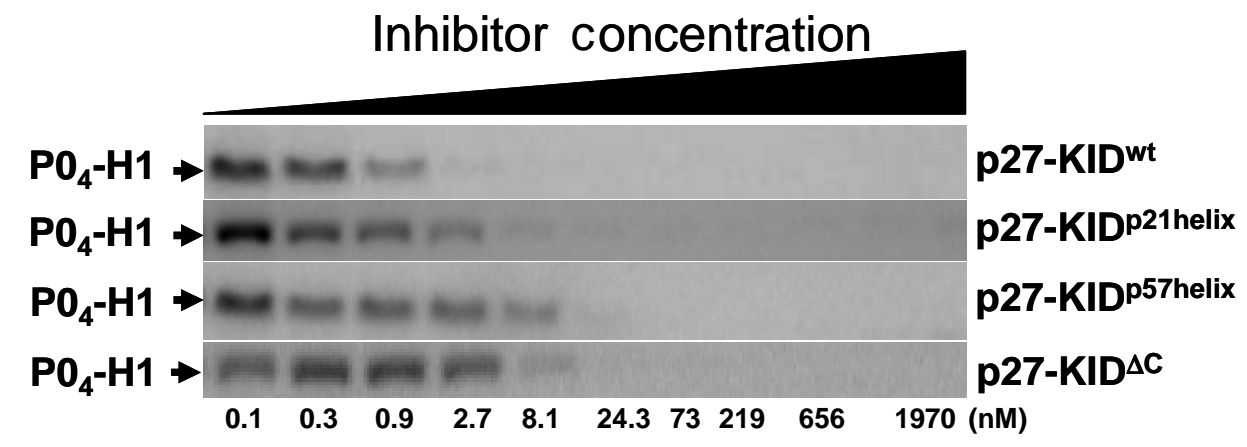

(b)

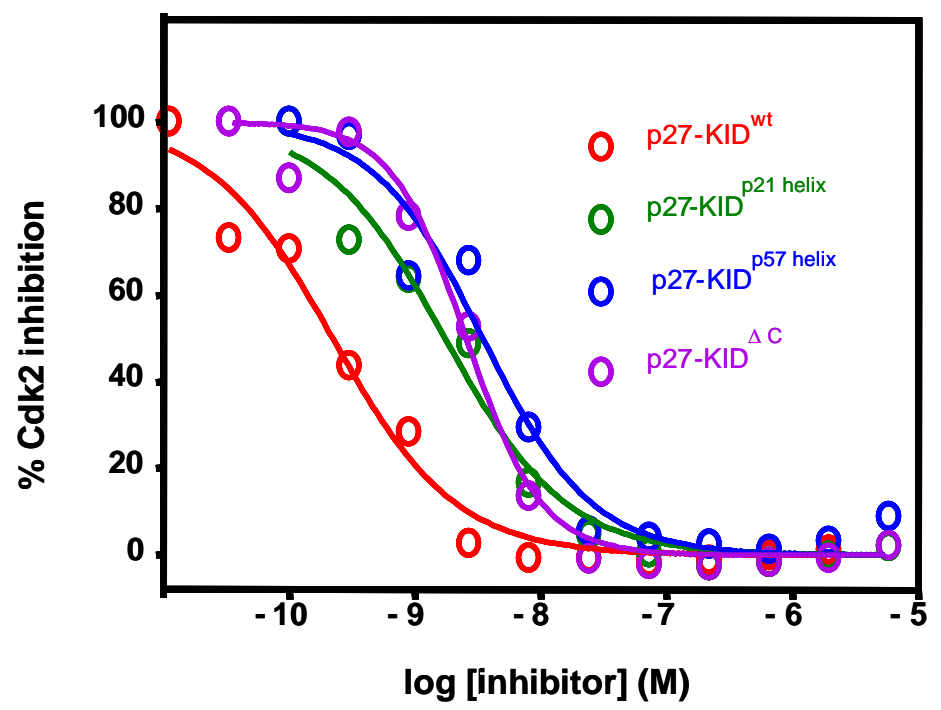

Figure 3.6 Inhibition of P-Cdk2/cyclin A kinase activity by the p27-KID variants. (a) SDS-PAGE gels showing the inhibition of the P-Cdk2/cyclin $A$ kinase by the p27-KID mutants. $\mathrm{PO}_{4}-\mathrm{H} 1$ is phophorylated histone $\mathrm{H} 1$ (b) Inhibition curves of the p27-KID mutants. 
complex. Even though the chimeric mutants are potent kinase inhibitors, their potencies are slightly decreased relative to the $\mathrm{p} 27-\mathrm{KID}^{\mathrm{wt}}$. The $\mathrm{p} 27-\mathrm{KID}^{\mathrm{p} 21 \mathrm{helix}}$

mutant is about 8 fold less potent than the $\mathrm{p} 27-\mathrm{KID}^{\mathrm{wt}}$, while the $\mathrm{p} 27-\mathrm{KID}^{\mathrm{p} 57 \mathrm{~h} \text { elix }}$ is about 16 fold less potent relative to the wild-type p27-KID.

The p27-KID ${ }^{\Delta C}$ mutant is also a potent inhibitor of the P-Cdk2/cyclin A kinase activity. This mutant has an $\mathrm{IC}_{50}$ value of $2.56 \mathrm{nM}$. Like the chimeric mutants, the p27-KID ${ }^{\Delta C}$ mutant is less potent than the p27-KID ${ }^{\text {wt }}$ (about 12 times less potent). This suggests that the conserved residues contribute marginally to the kinase inhibitory function of p27.

\subsection{Summary}

In this chapter, it has been demonstrated that the binding the D1 and D2 peptides to the P-Cdk2/cyclin A complex is less favorable relative to that of p27-KID ${ }^{\text {wt }}$. It has also been shown that the D2 peptide cannot completely inhibit the P-Cdk2/cyclin A kinase activity.

In addition, I have shown that the linker domains of the Cip/Kip proteins play similar structural roles, the differences in their sequences notwithstanding. The free energies of association of chimeric mutants (created by substituting the linker domain of p27 with p21 and p57 linker domains) with the P-Cdk2/cyclin A complex are very comparable to the free energy of association of wild-type p27-KID with the same complex. The melting temperatures of the ternary complexes formed by the chimeric mutants on binding the P-Cdk2/cyclin A complex are very similar to that of the ternary of the p27-KID ${ }^{\text {wt }}$. The chimeric 
mutants are potent inhibitors of the kinase activity of P-Cdk2/cyclin A, with $\mathrm{IC}_{50}$ values in the low $\mathrm{nM}$ range.

Data from the third study shows that three conserved residues within the linker domains of the Cip/Kip proteins do not contribute significantly to the Gibbs free energy of association in the binding of p27-KID to the P-Cdk2/cyclin A. However, the conserved residues contribute to the thermal stability of the ternary complex formed when p27-KID binds the P-Cdk2/cyclin A complex. Further, these residues contribute, albeit marginally, to the inhibition of the P-Cdk2/cyclin A kinase activity. 


\section{Chapter 4: Consequences of perturbation of the structure of the linker domain of p27}

The nascent helical structure of the linker domain is conserved amongst members of the Cip/Kip family of cyclin-dependent kinase inhibitors; however, its role in function is not known. To investigate the role of the nascent structure of the linker domain, mutants with higher or lower helical contents than the wild-type p27-KID in the linker domain were prepared and the consequences of the structure perturbations on the interaction of p27-KID with P-Cdk2/cyclin A were monitored. First, the solvent exposed residues within the linker domain were substituted with residues that either stabilize or destabilize helices. The secondary structures of the mutants were determined by CD. The consequences of the mutations on the thermodynamics of association of p27 and the P-Cdk2/cyclin A were analyzed by ITC. CD thermal denaturation studies were used to determine the thermal stability of the ternary complexes formed by p27

and its variants bound to P-Cdk2/cyclin A. The effects of the mutations on p27 function were determined by Cdk2 inhibition assays and cell cycle arrest analysis.

\subsection{Secondary structure analysis of the more and less helical p27-KID mutants}

The mutants selected for these studies were part of a larger library of mutants as listed in Table 2.2. Figure 4.1 shows the CD spectra of $\mathrm{p} 27-\mathrm{KID}^{\mathrm{wt}}$, 


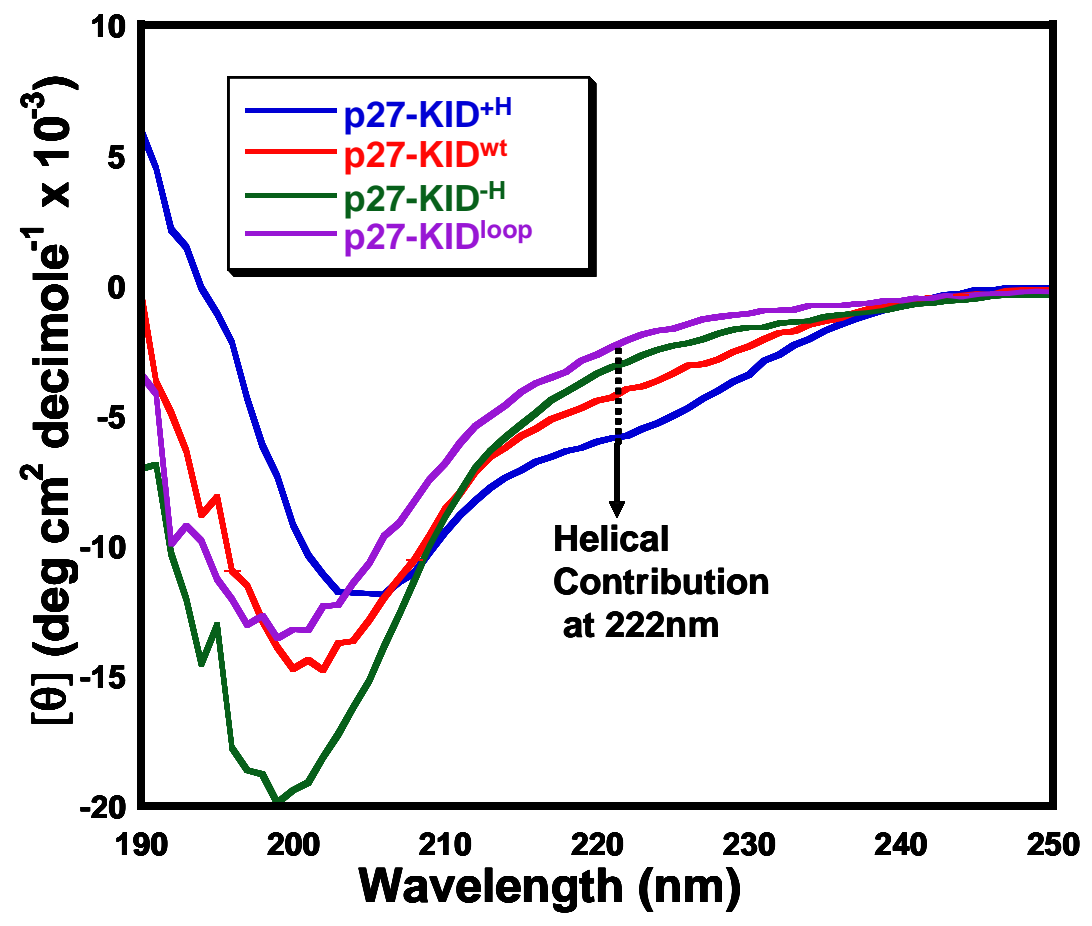

Figure 4.1 CD spectra of the p27-KID linker domain mutants. 
$\mathrm{p} 27-\mathrm{KID}^{+\mathrm{H}}, \mathrm{p} 27-\mathrm{KID}^{-\mathrm{H}}$, and $\mathrm{p} 27-\mathrm{KID}^{\mathrm{loop}}$ and the differences in their helical contents. p27-KID ${ }^{100 p}$ exhibits random coil structure, suggesting strongly that the helical contribution at $222 \mathrm{~nm}$ observed in the $\mathrm{p} 27-\mathrm{KID}^{\mathrm{wt}}$ is localized in the linker domain and not in D1 or D2. This is consistent with previous studies. ${ }^{197}$

\subsection{The free energy of the interaction of p27-KID with P-Cdk2/cyclin A does not decrease when the helical content in the linker domain of p27 is increased}

The thermodynamic binding parameters associated with the interaction of the p27-KID linker domain mutants and the P-Cdk2/cyclin A complex were determined by ITC. Figure 4.2 shows the ITC binding isotherms for these mutants. The interactions of the linker helix mutants with the P-Cdk2/cyclin A complex, involve dramatic folding-on-binding and are accompanied by large entropic costs, but not to the same extent as the p27-KID ${ }^{\mathrm{wt}}$. The observed $-\mathrm{T} \Delta \mathrm{S}$ values for the p27-KID ${ }^{w t}, p 27-K^{\prime} D^{+H}, p 27-K^{-H}$, and p27-KID ${ }^{\text {loop }}$ are $+32.2 \pm 1.2$ $\mathrm{Kcal} \mathrm{mol}^{-1},+22.3 \pm 2.5 \mathrm{Kcal} \mathrm{mol}^{-1},+19.0 \pm 1.0 \mathrm{Kcal} \mathrm{mol}^{-1}$, and $+20.0 \pm 1.4 \mathrm{Kcal}$ $\mathrm{mol}^{-1}$ respectively (Table 4.1). This suggests that $\mathrm{p} 27-\mathrm{KID}^{+\mathrm{H}}, \mathrm{p} 27-\mathrm{KID}^{-\mathrm{H}}$, and p27-KID ${ }^{\text {loop }}$ do not fold to the same extent upon binding to P-Cdk2/cyclin A as p27-KID ${ }^{\text {wt }}$. The folding of the mutants on binding the P-Cdk2/cyclin A complex results in a large number of favorable contacts. All the mutants release a large amount of heat when they bind the P-Cdk2/cyclin A complex. Nevertheless, the enthalpies of interaction for the mutants are smaller in magnitude relative to that for $\mathrm{p} 27-\mathrm{KID}^{\mathrm{wt}}$. The enthalpies of interaction for the $\mathrm{p} 27-\mathrm{KID}^{\mathrm{wt}}, \mathrm{p} 27-\mathrm{KID}^{+\mathrm{H}}$, 


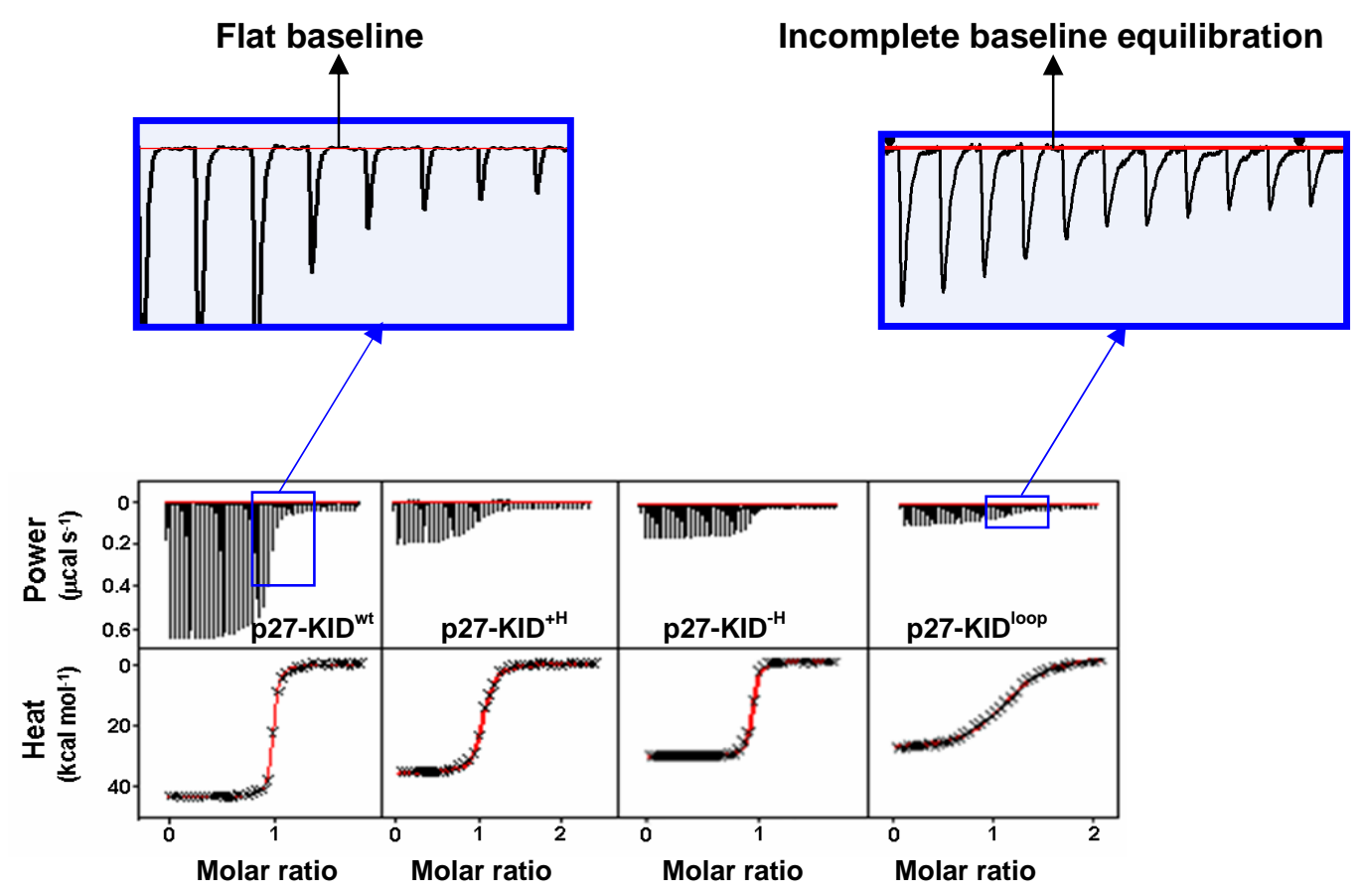

Figure 4.2 ITC binding isotherms for the interaction of the p27-KID mutants with the P-Cdk2/cyclin A complex. The transition regions for $\mathrm{p} 27-\mathrm{KID}^{\mathrm{wt}}$ and p27-KID ${ }^{\text {loop }}$ are expanded. 
Table 4.1 Thermodynamic binding parameters of the p27-KID mutants.

\begin{tabular}{|c|c|c|c|c|}
\hline Interaction & $\begin{array}{c}K_{\mathrm{d}} \\
(\mathrm{nM})\end{array}$ & $\begin{array}{c}\Delta \mathrm{G}^{\mathrm{a}} \\
\left(\mathrm{kcal} \mathrm{mol} \mathrm{mo}^{-1}\right)\end{array}$ & $\begin{array}{c}\Delta H \\
\left(k^{-1} \mathrm{~mol}^{-1}\right)\end{array}$ & $\begin{array}{c}-\mathrm{T} \Delta \mathrm{S}^{\mathrm{b}} \\
\left(\mathrm{kcal} \mathrm{mol}^{-1}\right)\end{array}$ \\
\hline $\mathrm{p} 27-\mathrm{KID}^{\mathrm{wt}}+\mathrm{P}-\mathrm{Cdk2} / \mathrm{cyclin} \mathrm{A}$ & $3.34 \pm 1.64$ & $-11.61 \pm 0.30^{c}$ & $-43.77 \pm 1.38$ & $+32.16 \pm 1.22$ \\
\hline $\mathrm{p} 27-\mathrm{KID}^{+\mathrm{H}}+\mathrm{P}-\mathrm{Cdk} 2 / \mathrm{cyclin} \mathrm{A}$ & $23.41 \pm 6.09$ & $-10.42 \pm 0.15$ & $-32.72 \pm 2.57$ & $+22.3 \pm 2.46$ \\
\hline $\mathrm{p} 27-\mathrm{KID}^{-\mathrm{H}}+\mathrm{P}-\mathrm{Cdk} 2 /$ cyclin A & $3.66 \pm 0.91$ & $-11.52 \pm 0.14^{c}$ & $-30.48 \pm 0.82$ & $+18.96 \pm 0.95$ \\
\hline $\mathrm{p} 27-\mathrm{KID}^{\mathrm{loop}}+\mathrm{P}-\mathrm{Cdk} 2 / \mathrm{cyclin} \mathrm{A}$ & $138.3 \pm 43.05$ & $-9.37 \pm 0.18$ & $-29.12 \pm 1.19$ & $+19.7 \pm 1.36$ \\
\hline
\end{tabular}

In all the experiments above, the stoichiometry of binding was determined as one (1), with experimental error within $10 \%$. The errors reported above are the standard deviations from the mean of three independent experiments.

${ }^{a}$ Calculated with the equation $\Delta \mathrm{G}=-\mathrm{RT} \ln \left(1 / K_{\mathrm{d}}\right)$.

${ }^{\mathrm{b}}$ Calculated with the equation $\Delta \mathrm{G}=\Delta \mathrm{H}-\mathrm{T} \Delta \mathrm{S}$.

${ }^{c}$ The $c$ values for these p27-KID variants are very high and the $\Delta G$ values reported here are taken as estimates of the upper limits of $K_{\mathrm{d}}$. Ideal c values for accurate determination of $K_{d}$, have a range of 1 to 500 . The $c$ values for the binding of $\mathrm{p} 27-\mathrm{KID}^{\mathrm{wt}}$ and $\mathrm{p} 27-\mathrm{KID}^{-\mathrm{H}}$ to P-Cdk2/cyclin A complex are 2212 and 1698 , respectively. 
$\mathrm{p} 27-\mathrm{KID}^{-\mathrm{H}}$, and p27-KID ${ }^{\text {loop }}$ are $-43.8 \pm 1.4 \mathrm{kcal} \mathrm{mol}^{-1},-32.7 \pm 2.6 \mathrm{kcal} \mathrm{mol}^{-1},-30.5$ $\pm 0.8 \mathrm{kcal} \mathrm{mol}^{-1}$, and $-29.1 \pm 1.2 \mathrm{kcal} \mathrm{mol}^{-1}$ in the order given. These enthalpies of interaction, for the wild-type and mutants, overcome the respective unfavorable interaction entropies resulting in overall favorable Gibbs free energies of interaction. $\Delta \mathrm{G}$ values of $-11.6 \pm 0.3 \mathrm{kcal} \mathrm{mol}^{-1},-10.4 \pm 0.2 \mathrm{kcal}$ $\mathrm{mol}^{-1},-11.5 \pm 0.1 \mathrm{kcal} \mathrm{mol}^{-1}$, and $-9.4 \pm 0.2 \mathrm{kcal} \mathrm{mol}^{-1}$ were obtained for the $\mathrm{p} 27-\mathrm{KID}^{\mathrm{wt}}, \mathrm{p} 27-\mathrm{KID}^{+\mathrm{H}}, \mathrm{p} 27-\mathrm{KID}^{-\mathrm{H}}$, and $\mathrm{p} 27-\mathrm{KID}^{\text {loop }}$, respectively. The $\Delta \Delta \mathrm{G}$ value for the p27-KID ${ }^{+H}$ with respect to the $\mathrm{p} 27-\mathrm{KID}^{\mathrm{wt}}$ is $+1.2 \mathrm{kcal} \mathrm{mol}^{-1}$ demonstrating that increasing the helical content of the linker domain does not make the interaction of $\mathrm{p} 27-\mathrm{KID}^{\mathrm{wt}}$ with $\mathrm{P}-\mathrm{Cdk} 2 /$ cyclin A more favorable; in fact, it actually makes it slightly less favorable.

\subsection{The ternary complexes formed by the linker helix mutants on binding P-Cdk2/cyclin A are thermodynamically less stable relative to that of p27-KID ${ }^{\text {wt }}$}

The P-Cdk2/cyclin A complex has a modest thermal stability with a melting temperature $\left(T_{\mathrm{m}}\right)$ of $54.7 \pm 0.6{ }^{\circ} \mathrm{C} .{ }^{216}$ When $\mathrm{p} 27-\mathrm{KID}^{\mathrm{wt}}$ binds to the $\mathrm{P}-\mathrm{Cdk} 2 / \mathrm{cyclin}$ A complex, the thermal stability of the ternary complex is dramatically increased to $82.3 \pm 0.5^{\circ} \mathrm{C}$. Figure 4.3 shows the thermal denaturation curves of the ternary complexes formed by the interaction of the p27-KID linker helix mutants and the P-Cdk2/cyclin A complex. The $T_{m}$ for values for complexes containing p27-KID ${ }^{+H}, \mathrm{p} 27-\mathrm{KID}^{-\mathrm{H}}$, and p27-KID ${ }^{\text {loop }}$ were $80.3 \pm 0.2^{\circ} \mathrm{C}, 78.5 \pm 0.6^{\circ} \mathrm{C}$, and $73.0 \pm 0.3^{\circ} \mathrm{C}$ respectively (Table 4.2). The results indicate that perturbation of 


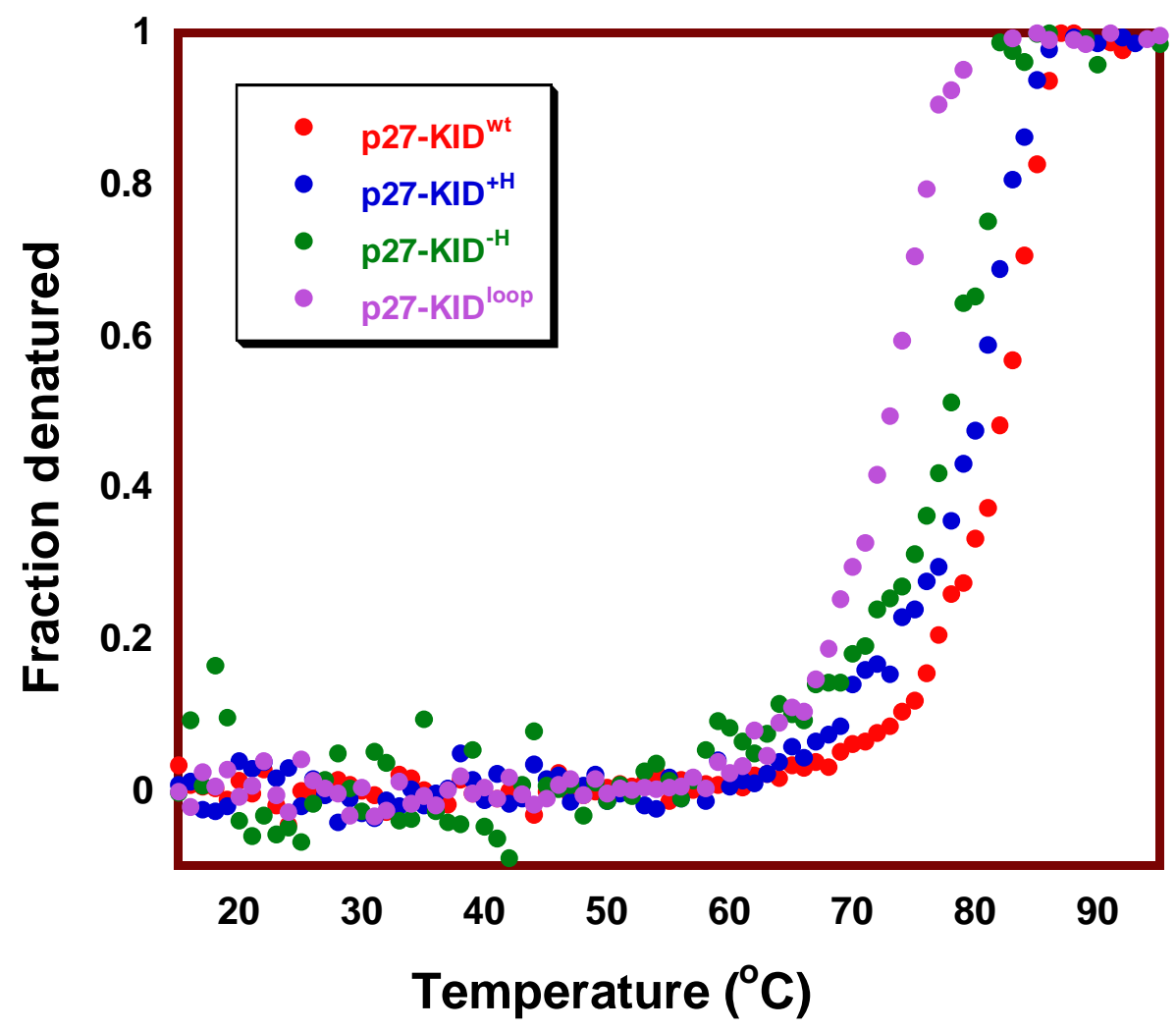

Figure 4.3 Thermal denaturation curves for the p27-KID variants. 
Table 4.2 Melting temperatures of the ternary complexes formed by the p27-KID mutants on binding the P-Cdk2/cyclin A complex.

\begin{tabular}{cc}
\hline Complex & $\boldsymbol{T}_{\mathrm{m}}\left({ }^{\circ} \mathrm{C}\right)$ \\
\hline $\mathrm{p} 27-\mathrm{KID}^{\mathrm{w}} / \mathrm{P}-\mathrm{Cdk} 2 /$ cyclin A & $82.3 \pm 0.5$ \\
$\mathrm{p} 27-\mathrm{KID}^{+\mathrm{H}} / \mathrm{P}-\mathrm{Cdk} 2 /$ cyclin A & $80.3 \pm 0.2$ \\
$\mathrm{p} 27-\mathrm{KID}^{-\mathrm{H}} / \mathrm{P}-\mathrm{Cdk} 2 /$ cyclin A & $78.5 \pm 0.6$ \\
$\mathrm{p} 27-\mathrm{KID}^{\mathrm{loop}} / \mathrm{P}-\mathrm{Cdk} 2 /$ cyclin A & $73.0 \pm 0.3$ \\
\hline
\end{tabular}


the helical content of the linker domain has a net effect of decreasing the thermal stabililty of the ternary complexes. Reducing the helical content $\left(\mathrm{p} 27-\mathrm{KID}^{-\mathrm{H}}\right)$ or completely eliminating the nascent helical structure (p27-KID ${ }^{\text {loop }}$ ) has a greater impact on the thermal stability than increasing the helical content $\left(\mathrm{p} 27-\mathrm{KID}^{+\mathrm{H}}\right)$. The decrease in the melting temperature of the ternary complex containing the p27-KID ${ }^{+H}$ mutant relative to that of $\mathrm{p} 27-\mathrm{KID}^{\mathrm{wt}}$ indicates that restricting the dynamics of the linker domain by stabilizating its helical structure has a destabilizing effect on the p27-KID ${ }^{\mathrm{wt}}$ ternary complex. On the other hand, the $T_{m}$ 's obtained for the $\mathrm{p} 27-\mathrm{KID}^{-\mathrm{H}}$ and $\mathrm{p} 27-\mathrm{KID}^{\text {loop }}$ mutants suggest that excessive flexibility of the linker domain is also destabilizing.

\subsection{The linker helix mutants are less potent inhibitors of P-Cdk2/cyclin A kinase activity relative to $\mathrm{P27}-\mathrm{KID}^{\mathrm{wt}}$}

In the last chapter, it was shown that p27-KID is a potent inhibitor of the kinase activity of the P-Cdk2/cyclin A complex and that a Cdk binding domain peptide (D2) cannot completely inhibit kinase activity. The D2 peptide partially inhibits P-Cdk2/cyclin A kinase activity with an $\mathrm{IC}_{50}$ of about $125 \mathrm{nM}$. However, the D2 peptide fails to completely inhibit P-Cdk2/cyclin A with $25 \%$ residual kinase activity observed even at inhibitor peptide concentrations as high as 17 $\mu \mathrm{M}$. These data suggest that the Cdk binding domain requires the other domains of the KID (the cyclin binding domain and the linker domain) for effective inhibition. The structure of the p27-KID/Cdk2/cyclin A complex shows that the the LH domain of p27 is an $\alpha$-helix in this ternary complex. ${ }^{104}$ However, the role 
of the helical propensity of the LH domain of unbound p27 in binding and inhibition is not known. I performed kinase inhibition assays to uncover the contribution of the structure of the linker domain to Cdk inhibition.

All of the linker helix mutants inhibit kinase activity in the low nanomolar range. Nevertheless, none of the mutants is more potent than the wild-type KID. Figure 4.4 shows the raw inhibition data and normalized inhibition curves for the p27-KID mutants. The values for the p27-KID ${ }^{w t}, p 27-K D^{+H}, p 27-K^{-H}{ }^{-H}$, and p27-KID ${ }^{\text {loop }}$ were $293.8 \mathrm{pM}, 5.1 \mathrm{nM}, 353.0 \mathrm{pM}$, and $16.0 \mathrm{nM}$ (Table 4.3), respectively. The results obtained for the $\mathrm{p} 27-\mathrm{KID}^{\mathrm{loop}}$ mutant are very interesting because they show that a KID molecule formed by coupling the cyclin and Cdk binding domains with an unstructured flexible linker can inhibit the P-Cdk2/cyclin A cyclin complex with an $\mathrm{IC}_{50}$ as low as $16 \mathrm{nM}$. Increasing the helical content of the linker domain decreases inhibition potency relative to the $\mathrm{p} 27-\mathrm{KID}^{\mathrm{wt}}$. The p27-KID ${ }^{+H}$ variant is about 17 times less potent than the wild-type KID in inhibiting P-Cdk2/cyclin A kinase activity. However, reducing the helical content $\left(p 27-K D^{-H}\right)$ does not diminish the inhibition potency of the KID to the same extent. The $\mathrm{p} 27-\mathrm{KID}^{-\mathrm{H}}$ is almost as potent as the wild-type KID. Since the linker domain does not have extensive interactions with either cyclin A or P-Cdk2, its effects on phosphorylation are most likely a consequence of the role it plays in the folding of the Cdk binding domain.

It should be noted that the disparity between the $\mathrm{IC}_{50}$ value, $(16 \mathrm{nM})$, and $K_{d}$ value, $(138 \mathrm{nM})$, for the p27-KID ${ }^{\text {loop }}$ mutant is likely due to kinetic effects. In Figure 4.2, the expanded regions compare the raw data for the ITC binding 
(a)

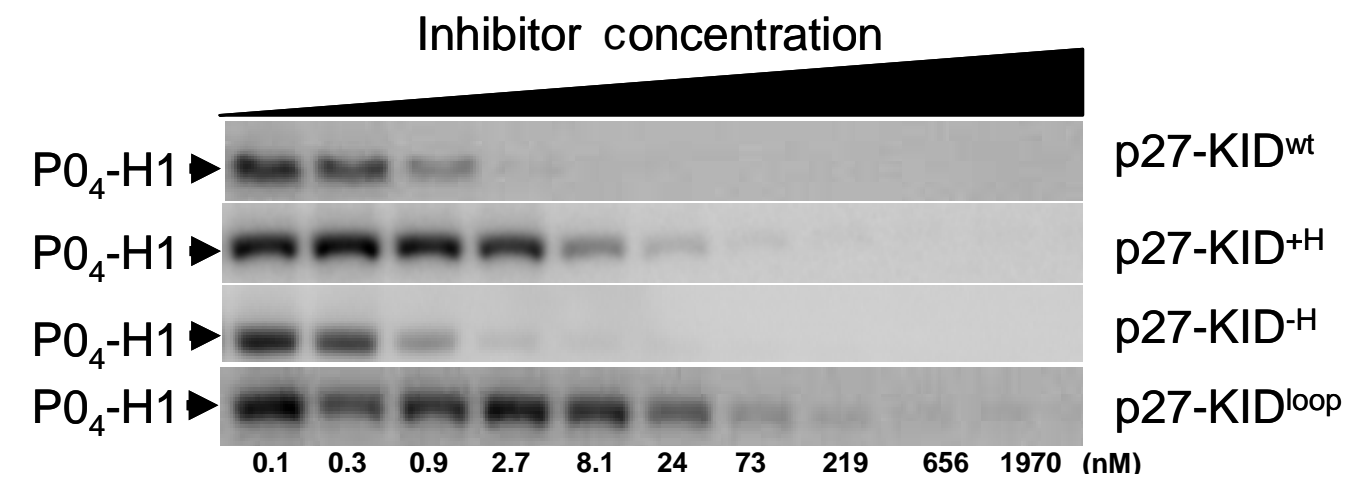

(b)

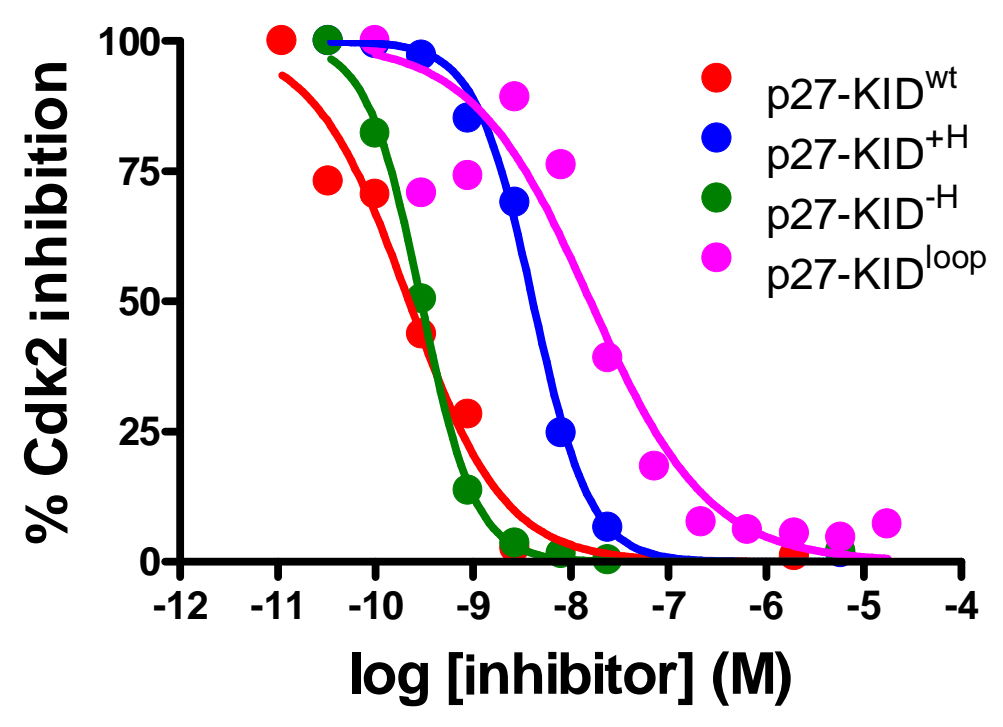

Figure 4.4 P-Cdk2/cyclin A kinase inhibition by the p27-KID mutants. (a) Gels showing kinase inhibition the p27-KID mutants. (b) Inhibition curves of the p27-KID variants. 
Table 4.3 Inhibition potencies of the p27-KID mutants.

\begin{tabular}{ccc}
\hline Variant & IC $_{50}(\mathbf{n M})$ & 95\% confidence intervals of $\mathbf{I C}_{\mathbf{5 0}}(\mathbf{n M})^{\mathbf{a}}$ \\
\hline $\mathrm{p} 27-\mathrm{KID}^{\mathrm{wt}}$ & $293.8 \mathrm{pM}$ & $0.17-0.28$ \\
$\mathrm{p} 27-\mathrm{KID}^{+H}$ & $5.06 \mathrm{nM}$ & $3.3-5.1$ \\
$\mathrm{p} 27-\mathrm{KID}^{-H}$ & $353 \mathrm{pM}$ & $0.26-0.33$ \\
$\mathrm{p} 27-\mathrm{KID}^{\text {loop }}$ & $15.95 \mathrm{nM}$ & $9.9-25.7$ \\
\hline
\end{tabular}

${ }^{a}$ Error is reported as the $95 \%$ confidence interval of the $\mathrm{IC}_{50}$ from a curve fit of a triplicate data set. 
isotherms for $\mathrm{p} 27-\mathrm{KID}^{\mathrm{wt}}$ and $\mathrm{p} 27-\mathrm{KID}^{\mathrm{loop}}$. From the figure it is clear that unlike the p27-KID wt which equilibrates to a completely flat baseline between injections, the injection peaks of the p27-KID ${ }^{\text {loop }}$ mutant do not fully equilibrate to a flat baseline in the time between injections. The equilibration time between injections is 5 minutes. On the other hand, during the kinase assays, P-Cdk2/cyclin A was mixed with p27-KID ${ }^{\text {loop }}$ and incubated for 1 hour prior to addition of ATP. It is conceivable that in an hour the binding reaction reaches equilibrium explaining the difference between $K_{\mathrm{d}}$ and $\mathrm{IC}_{50}$. Such a long equilibration time would not be practical in an ITC experiment because it would require a total experiment time of at least 40 hours. In addition, the P-Cdk2/cyclin A complex would precipitate if kept at $25^{\circ} \mathrm{C}$ for such an extended period of time making such an experiment unfeasible.

\subsection{Perturbation of the structure of the linker domain is deleterious to p27 function in cells}

Previous studies have shown that up-regulation of p27 causes cell cycle arrest during the $\mathrm{G} 1 / \mathrm{S}$ cell cycle transition. ${ }^{111}$ The effects of increasing or decreasing the helical content of the linker domain on cell cycle arrest were probed by over-expressing the p27 mutants in mouse fibroblast cells immortalized using the NIH 3 T3 protocol. ${ }^{232}$ The linker LH domain mutants were expressed in the context of the full-length p27 protein. The level of protein expression was evaluated by measuring the GFP fluorescence of NIH 3T3 cells using fluorescence activated cell sorting (FACS) analysis (Figure A.7). The DNA 
content of cells expressing the greatest amount of GFP (top 10\%) was measured for cell cycle analysis. Relative to a vector only negative control, the flp2 $7^{\mathrm{wt}}$, caused cell cycle arrest and reduced the percentage of cells is S-phase from $28.4 \%$ to $14 \%$ (Table 4.4, Figure A.8). The mutant with the stabilized helix, flp2 $7^{+H}$, also caused cell cycle arrest. However, its cell cycle arrest capacity relative to flp $27^{\mathrm{wt}}$ is diminished. $18.2 \%$ of the cells progressed to S-phase when flp2 $7^{+\mathrm{H}}$ mutant was over-expressed. The greatest effects on cell cycle arrest were observed when the structure of the linker domain was either destabilized $\left(\mathrm{flp} 27^{-\mathrm{H}}\right.$ ) or completely eliminated (flp2 $7^{\text {loop }}$ ). When either flp2 $7^{-\mathrm{H}}$ or flp2 $7^{\text {loop }}$ was over-expressed, $28.5 \%$ and $30.5 \%$ of the cells respectively progressed to the S-phase from the G1 phase. Compared to the vector only negative control (28.4\%), the flp2 $7^{-\mathrm{H}}$ and the flp2 $7^{\text {loop }}$ mutants do not appear to cause cell cycle arrest.

\subsection{Summary}

In this chapter, the thermodynamic and functional effects of perturbations of the structure of the linker domain of p27 have been reported. There was a decrease in the enthalpy of association of p27 with the P-Cdk2/cyclin A complex when the structure of the linker domain was perturbed. However, this reduction in enthalpy was compensated for by a proportional reduction in the entropic cost associated with the folding of the p27-KID mutants on binding the P-Cdk2/cyclin A complex. This compensation caused the Gibbs free energies of association of all the $\mathrm{p} 27-\mathrm{KID}$ variants to vary within a very narrow range. The ternary 
Table 4.4 Cell cycle analysis of cells.

\begin{tabular}{ccccccc}
\hline $\begin{array}{c}\text { Cell cycle } \\
\text { phase }\end{array}$ & No plasmid & $\begin{array}{c}\text { Empty vector } \\
\text { (GFP only) }\end{array}$ & $\mathbf{p 2 7} 7^{\mathbf{w t}}$ & $\mathbf{p 2 7}^{+\mathbf{H}}$ & $\mathbf{p 2 7} 7^{-\mathbf{H}}$ & $\mathbf{p 2 7}^{\text {loop }}$ \\
\hline G0/G1 & $55.3 \pm 2.6$ & $22.2 \pm 2.1$ & $57.9 \pm 0.4$ & $48.5 \pm 3.0$ & $38.7 \pm 3.2$ & $39.9 \pm 2.3$ \\
$\mathbf{S}$ & $34.4 \pm 2.1$ & $28.4 \pm 2.4$ & $14 \pm 1.0$ & $18.2 \pm 1.7$ & $28.1 \pm 1.1$ & $30.5 \pm 1.2$ \\
$\mathbf{G 2 / M}$ & $10.3 \pm 1.3$ & $49.5 \pm 2.6$ & $28.2 \pm 0.6$ & $33.3 \pm 1.4$ & $33.1 \pm 3.0$ & $29.6 \pm 1.5$ \\
\hline
\end{tabular}

The values represent the percentage of cells at the various phases of the cell cycle. Triplicate experiments were done for each control and p27-KID variant. 
complexes formed when the mutants bind to the P-Cdk2/cyclin A complex were thermally less stable than that formed with wild-type p27-KID. All the mutants were less potent inhibitors of the P-Cdk2/cyclin A complex relative to the p27-KID ${ }^{\text {wt }}$. While enhancing the helical structure of the linker domain has a slight negative impact on the ability of p27 to cause cell cycle arrest, destabilizing or replacing this structure with a loop is deleterious to p27 function. 


\section{Chapter 5: Discussion}

\subsection{The linker domain of $p 27$ is necessary for function}

The studies of the interactions of D1 and D2 with P-Cdk2/cyclin A support my hypothesis that the linker domain of p27 is necessary for function. The crystal structure of the $\mathrm{p} 27 / \mathrm{Cdk} 2 /$ cyclin A complex shows that the linker domain makes few contacts with the Cdk2/cyclin A complex. It follows that the sum of the enthalpy of association of D1 with the P-Cdk2/cyclin A complex and the enthalpy of association of D2 with the same complex should either be equal to or slightly greater than (more positive) the interaction of p27 with the P-Cdk2 cyclin A complex. However, the total observed enthalpy for the D1 and D2 interactions $\left(-47.69 \mathrm{kcal} \mathrm{mol}^{-1}\right)$ with the binary complex was less than (more negative) that for p27-KID ${ }^{\text {wt }}\left(-43.77 \mathrm{kcal} \mathrm{mol}^{-1}\right)$. This suggests that even though D1 and D2 bind to the P-Cdk2/cyclin A complex, one or both domains does (do) not make exactly the same contacts with P-Cdk2/cyclin A as they do in the context of p27-KID ${ }^{\text {wt }}$. A previous study reported by our laboratory suggests that it is unlikely that D1 misfolds. ${ }^{197}$ First, the binding of D1 to the cyclin does not involve large conformational changes which would require overcoming many local free energy barriers. Secondly, the enthalpy of the interaction D1 to the P-Cdk2/cyclin A complex $\left(-16.1 \pm 0.2 \mathrm{kcal} \mathrm{mol}^{-1}\right)$ is very similar to that of the $\mathrm{p} 27-\mathrm{KID}^{\mathrm{wt}}$ on binding

cyclin A $\left(-17.2 \pm 0.3 \mathrm{kcal} \mathrm{mol}^{-1}\right) .{ }^{197}$ This suggests that favorable contacts made in both cases are very similar, if not identical. 
On the other hand, the binding of D2 to the Cdk2 subunit of the kinase complex involves a number of significant conformational changes and dramatic folding of p27-KID and remodeling within Cdk2. This makes it likely that the D2 peptide does not make the same contacts with Cdk2 as does the D2 domain in the context of $\mathrm{p} 27-\mathrm{KID}^{\mathrm{wt}}$. The results of the kinase inhibition assays confirm this (Figure 3.5). ITC shows that D2 binds to the kinase complex with reduced affinity $\left(K_{d}=82.96 \pm 17.89 \mathrm{nM}\right)$. However, D2 is unable to completely inhibit Cdk2 kinase activity. Differences in the dynamics of bound D2 peptide relative to that of bound D2 in the context of the p27-KID may account for the differences in Cdk2 kinase inhibition observed for the D2 peptide and p27-KID ${ }^{\text {wt }}$. I propose that the D2 peptide bound to Cdk2 is more dynamic than Cdk2-bound D2 in the context of the p27-KID ${ }^{\text {wt }}$. The more dynamic nature of the Cdk2-bound D2 peptide allows some ATP molecules to access to the ATP-binding pocket thereby enabling the $25 \%$ residual kinase activity observed during the kinase inhibition assays. I further propose that the coupling of D2 to D1 by the linker domain in p27-KID ${ }^{\text {wt }}$ restricts the motion of D2 on binding Cdk2. Because of the motional restriction, the Cdk-binding domain of $\mathrm{p} 27-\mathrm{KID}^{\mathrm{wt}}$ completely occludes the ATP binding pocket thereby blocking ATP access and ipso facto completely inhibiting Cdk2 kinase activity.

In addition to facilitating kinase inhibition, the coupling of D1 and D2 by the linker domain makes the interaction of $\mathrm{p} 27-\mathrm{KID}^{\mathrm{wt}}$ with $\mathrm{P}-\mathrm{Cdk2} / \mathrm{cyclin} \mathrm{A}$ more favorable than the interactions of either D1 or D2 peptides with the same complex. The $\Delta \mathrm{G}$ of the binding of $\mathrm{p} 27-\mathrm{KID}^{\mathrm{wt}}$ to P-Cdk2/cyclin A complex $(-11.61$ 
$\left.\pm 0.30 \mathrm{kcal} \mathrm{mol}^{-1}\right)$ is more favorable relative to that of either D1 $(-10.06 \pm 0.07$ $\left.\mathrm{kcal} \mathrm{mol}{ }^{-1}\right)$, or D2 $\left(-9.66 \pm 0.12 \mathrm{kcal} \mathrm{mol}^{-1}\right)$. This raises a question as to how the coupling of D1 and D2 by the linker domain makes the binding of $\mathrm{p} 27-\mathrm{KID}^{\mathrm{wt}}$ to the P-Cdk2/cyclin A more favorable than the binding of D1 and D2 peptides to the same complex.

For the binding of $\mathrm{p} 27-\mathrm{KID}^{\mathrm{wt}}$ to $\mathrm{P}-\mathrm{Cdk} 2 / \mathrm{cyclin} \mathrm{A}$ to be more favorable than the binding of either D1 or D2, the coupling of D1 and D2 with the linker domain has to either cause net increase the amount of heat released (net increase in the enthalpy of binding) or increase the overall disorder of the system (net increase in entropy). From the structure of the p27-KID/Cdk2/cyclin A it is evident that the linker domain makes very few contacts with either Cdk2 or cyclin A. It is therefore unlikely that the linker domain decreases the free energy of the binding of $\mathrm{p} 27-\mathrm{KID}^{\mathrm{wt}}$ to $\mathrm{P}-\mathrm{Cdk2/cyclin} \mathrm{A}$ (relative to the binding of D1 and D2 peptides to P-Cdk2/cyclin A) by causing a net increase in the enthalpy of binding.

The other explanation is that the coupling of D1 and D2 with the linker domain decreases the free energy of binding of $p 27-K D^{w t}$ to $P-C d k 2 / c y c l i n ~ A$, relative to the binding of $D 1$ and $D 2$ to the same complex, by causing a net increase in the entropy of binding. The net change in the entropy of binding is determined by two components: (i) the entropy of water resulting from the burial of hydrophobic side-chains (favorable entropy); and (ii) the conformational entropy that results from loss of translational and rotational degrees during protein folding (unfavorable entropy) and the motion of the protein after binding (favorable entropy). ${ }^{233,234}$ Since there is no significant burial of hydrophobic 
residues within the linker domain when $\mathrm{p} 27-\mathrm{KID}^{\mathrm{wt}}$ binds to Cdk2/cyclin $A$, the contribution of the entropy of water toward a net entropy increase should be minimal. This suggests that the net increase in entropy contribution of the linker domain toward the binding of $\mathrm{p} 27-\mathrm{KID}^{\mathrm{wt}}$ to $\mathrm{P}-\mathrm{Cdk} 2 / \mathrm{cyclin} \mathrm{A}$ is caused by a net positive change in conformational entropy. This positive change in entropy indicates that the contribution of the dynamics (positive entropy change) of the linker domain of p27 when bound to P-Cdk2/cyclin A is greater than the entropic contribution of the folding of the linker domain (negative entropy change). This is consistent with recent results from our laboratory (unpublished results). NMR analysis of p27-KID in complex with Cdk2/cyclin A has showed that the linker domain is dynamic even when p27 is bound to Cdk2/cyclin A. The same has also been observed for the linker domain of p21-KID in a p21-KID/Cdk2/cyclin A complex (unpublished results). I propose that the dynamic nature of the linker domain of the Cip/Kip proteins contributes significantly to the free energy of their association with Cdk complexes.

\subsection{The linker domains of the Cip/Kip proteins play similar structural roles despite their sequence differences}

The sequences of the linker domains of the Cip/Kip proteins are very variable but have a conserved helical structure. An analysis of the linker domain of each Cip/Kip protein in different species shows very high sequence conservation. It is curious why nature has selected for disparate sequences in the linker domains of the Cip/Kip proteins. It is reasonable to argue that if just a 
helical structure was required to couple D1 and D2, then any sequence that could adopt a helical structure could have been selected for; but, this is clearly not the case. This may indicate that these sequences may play well defined roles in the interactions of the Cip/Kip proteins with Cdk's. This poses a question as to whether the different sequences of the linker domains of the Cip/Kip proteins confer each protein different thermodynamic and functional properties when they bind to their Cdk targets.

ITC studies show that the free energies of association of the chimeric mutants on binding the P-Cdk2/cyclin A complex are very similar. The $\mathrm{p} 27-\mathrm{KID}^{\mathrm{wt}}$ has a $\Delta \mathrm{G}$ value of $-11.61 \mathrm{kcal} \mathrm{mol}^{-1}$, which is very close to the $-11.7 \pm 0.27 \mathrm{kcal}$ $\mathrm{mol}^{-1}$ and $-12.71 \pm 0.17 \mathrm{kcal} \mathrm{mol}^{-1}$ obtained for the $\mathrm{p} 27-\mathrm{KID}^{\mathrm{p} 21 \mathrm{helix}}$ and the p27-KID ${ }^{\text {p57helix }}$, respectively. This shows that the three linker domains despite their differences in sequence contribute very similarly to the free energy of association of the Cip/Kip proteins with the P-Cdk2/cyclin A complexes. One difference observed in the thermodynamic parameters is the magnitude of the enthalpy of binding.

The chimeric mutants exhibit decreased value of the enthalpy of binding relative to that for $\mathrm{p} 27-\mathrm{KID}^{\mathrm{wt}} \cdot \mathrm{p} 27-\mathrm{KID}^{\mathrm{p} 21 \mathrm{helix}}$ and $\mathrm{p} 27-\mathrm{KID}^{\mathrm{p} 57 \mathrm{~h} \text { elix }}$ exhibit enthalpies of $-27.94 \pm 1.26 \mathrm{kcal} \mathrm{mol}^{-1}$ and $-31.15 \pm 0.51 \mathrm{kcal} \mathrm{mol}^{-1}$, respectively, in comparison to $\mathrm{p} 27-\mathrm{KID}^{\mathrm{wt}}$ which has an enthalpy of $-43.77 \mathrm{kcal} \mathrm{mol}^{-1}$ on binding P-Cdk2/cyclin A. The kinase assay results show that these chimeric variants are potent inhibitors albeit slightly less potent than $\mathrm{p} 27-\mathrm{KID}^{\mathrm{wt}}$. This shows that D2 in the chimeric mutants binds to Cdk2 and is folded in a conformation very similar to 
that of $\mathrm{D} 2$ of the $\mathrm{p} 27-\mathrm{KID}^{\mathrm{wt}}$. It is unlikely that $\mathrm{D} 1$ of the chimeric mutants is not binding since the free energies of association of the chimeric mutants with P-Cdk2/cyclin A are more comparable to the free energy of the association of p27-KID ${ }^{\text {wt }}$ and P-Cdk2/cyclin A than to the free energy of association of either the D2 peptide and the binary complex or p27-KID with monomeric Cdk2. ${ }^{197}$ If D1 was not binding, the free energies of the association of the chimeric mutants and P-Cdk2/cyclin A should reflect binding only to Cdk2. This suggests that the enthalpy decrease observed in the ITC experiments is likely a result of the linker domains (since D1 and D2 bind) of the chimeric mutants not having exactly the same favorable interactions on binding P-Cdk2/cyclin A as p27-KID ${ }^{\text {wt }}$. While the loss of enthalpy contributes unfavorably to the free energy of interaction, it is countered by a favorable decrease in the entropic penalty associated with the folding on binding. The $-\mathrm{T} \Delta S$ values for the $\mathrm{p} 27-\mathrm{KID}^{\mathrm{p} 21 \text { helix }}$ and the $\mathrm{p} 27-\mathrm{KID}^{\mathrm{p} 57 \text { helix }}$ are $+16.24 \pm 1.29 \mathrm{kcal} \mathrm{mol}^{-1}$ and $+18.44 \pm 0.35 \mathrm{kcal} \mathrm{mol}^{-1} 1$ respectively, in comparison to the greater entropic cost of $+32.16 \pm 1.22 \mathrm{kcal} \mathrm{mol}^{-1}$ of the interaction of $\mathrm{p} 27-\mathrm{KID}^{\mathrm{wt}}$ with $\mathrm{P}-\mathrm{Cdk} 2 / \mathrm{cyclin} \mathrm{A}$. The proportional decrease in the entropic penalty effectively compensates for the enthalpic loss in the chimeric mutants resulting in free energy values very close to that of the $\mathrm{p} 27-\mathrm{KID}^{\mathrm{wt}}$. The chimeric mutants exhibit an entropy-enthalpy compensation phenomenon that makes their interaction with the P-Cdk2/cyclin A complex favorable.

It has been shown that the binding of the $\mathrm{p} 27-\mathrm{KID}^{\mathrm{wt}}$ to the $\mathrm{P}-\mathrm{Cdk} 2 / \mathrm{cyclin} \mathrm{A}$ complex greatly stabilizes the formed ternary complex. ${ }^{216}$ The melting temperature of the P-Cdk2/cyclin A complex , which is only $54.7 \pm 0.6{ }^{\circ} \mathrm{C}$, 
increases to $82.3 \pm 0.5{ }^{\circ} \mathrm{C}$ when the $\mathrm{p} 27-\mathrm{KID}^{\mathrm{wt}}$ binds. The binding of the chimeric mutants stabilize the binary complex to extents very similar to that of the p27-KID ${ }^{w t}$. The ternary complexes of $p 27-K D^{p 21 \text { helix }}$ and $p 27-K D^{p 57 h e l i x}$ have melting temperatures of $80.7 \pm 0.2{ }^{\circ} \mathrm{C}$ and $80.1 \pm 0.1{ }^{\circ} \mathrm{C}$ respectively. This suggests that the non-conserved residues in the linker domain contribute minimally to the stability of the ternary complexes formed when the Cip/Kip proteins bind the binary complex. The thermal denaturation data taken together with the ITC and kinase inhibition assay data suggest that the linker domains of the Cip/Kip proteins, despite their sequence differences, play very similar structural roles in binding to Cdk2/cyclin A.

\subsection{The role of the conserved residues in the linker domain of Cip/Kip proteins}

Only 3 (Glu39, Leu41 and Arg43) out of the 22 residues in the linker domain are conserved. I mutated these residues to glycines to probe their role in the interaction of the p27-KID with the P-Cdk2/cyclin A complex. The thermodynamic parameters obtained from ITC are very similar to those of the p27-KID ${ }^{\text {wt }}$. There is a difference in the enthalpy of p27-KID ${ }^{\Delta C}$ mutant and p27-KID ${ }^{\mathrm{wt}}$. The enthalpy of association of $\mathrm{p} 27-\mathrm{KID}^{\Delta \mathrm{C}}$ is $-39.65 \pm 0.13 \mathrm{kcal} \mathrm{mol}^{-1}$, a decrease of $4.12 \mathrm{kcal} \mathrm{mol}^{-1}$ relative to that of the $\mathrm{p} 27-\mathrm{KID}^{\mathrm{wt}}$. This indicates that the conserved residues contribute about $4 \mathrm{kcal} \mathrm{mol}^{-1}$ to the enthalpy of interaction of the $\mathrm{p} 27-\mathrm{KID}^{\mathrm{wt}}$ with P-Cdk2/cyclin A. ${ }^{104}$ This difference is most likely caused by the mutation of Leu41 to Gly. In the p27/Cdk2/cyclin A structure, 
Leu41 is buried into the p27-KID-cyclin A interface and mutating it to a smaller side chain, Gly, reduces the buried surface area resulting in a reduction of the amount of heat released. The other two conserved residues Glu39 and Arg43 are largely solvent exposed. Their mutation to Gly's probably does not have a great effect on the enthalpy. However, the Gly mutations may affect the dynamics of the linker helix by making it more flexible since the glycine side chain can access a broad range of conformational states. Like the chimeric mutants, this slight loss of enthalpy is compensated for and the free energy of association is the same as that of the p27-KID ${ }^{\text {wt }}$ within standard error. The free energy of association of $\mathrm{p} 27-\mathrm{KID}^{\Delta \mathrm{C}}$ with the binary complex is $-11.89 \mathrm{Kcal} \mathrm{mol}^{-1}$.

Even though the thermodynamic parameters associated with p27-KID ${ }^{\Delta C}$ mutant are very similar to those associated with $\mathrm{p} 27-\mathrm{KID}^{\mathrm{wt}}$, the ternary complex this mutant forms on binding the binary complex is significantly less stable than that formed by $\mathrm{p} 27-\mathrm{KID}^{\mathrm{wt}}$. The binding of $\mathrm{p} 27-\mathrm{KID}^{\Delta \mathrm{C}}$ is associated with a $T_{\mathrm{m}}$ of $74.1 \pm 0.6^{\circ} \mathrm{C}$, about $8^{\circ} \mathrm{C}$ less than that associated with $\mathrm{p} 27-\mathrm{KID}^{\mathrm{wt}}$. This demonstrates that the conserved residues contribute significantly to the stability of the ternary complex.

The $\mathrm{p} 27-\mathrm{KID}^{\Delta \mathrm{C}}$ mutant is slightly less potent than the p27-KID ${ }^{\mathrm{wt}}$. The $\mathrm{IC}_{50}$ of this mutant is $2.56 \mathrm{nM}$ while the $\mathrm{p} 27-\mathrm{KID}^{\mathrm{wt}}$ has an $\mathrm{IC}_{50}$ value of $0.22 \mathrm{nM}$. This clearly indicates that the effects of mutations which are very close to D1 are propagated along the length of the helix and are able to affect the interaction D2 and Cdk2 resulting in the decrease in inhibition potency. This attests to the dynamic nature of the linker domain of the Cip/Kip proteins. In summary, the 
conserved residues in the linker domains of the Cip/Kip proteins contribute to the enthalpy of binding of p27-KID to P-Cdk2/cyclin A, the kinase activity of p27-KID ${ }^{\mathrm{wt}}$, and also play a role in increasing the thermal stability of the ternary complex formed on when p27 binds P-Cdk2/cyclin A.

\subsection{Biophysical and functional effects of increasing, decreasing, or eliminating the helical structure of the linker domain of p27}

The nascent helical structure of the linker domain is conserved amongst members of the Cip/Kip family. I probed the consequences of perturbing this conserved structure on the biophysical and functional properties of p27 by studying the interactions of p27 mutants, in which the helical content of the linker domain was either increased $\left(\mathrm{p} 27-\mathrm{KID}^{+\mathrm{H}}\right)$, decreased $\left(\mathrm{p} 27-\mathrm{KID}^{-\mathrm{H}}\right)$, or completely eliminated (p27-KID ${ }^{\text {loop}}$ ), with the P-Cdk2/cyclin A. The binding of p27 to Cdk2/cyclin A is associated with a great entropic cost. I hypothesized that the preformed helix in the linker domain of p27 decreases this entropic cost.

Results from the ITC analysis of the binding of p27-KID ${ }^{\mathrm{wt}}$, p27-KID ${ }^{+\mathrm{H}}$, and $\mathrm{p} 27-\mathrm{KID}^{-\mathrm{H}}$ to $\mathrm{P}-\mathrm{Cdk} 2 /$ cyclin A disprove this hypothesis. While the relative $-\mathrm{T} \Delta \mathrm{S}$ values for the binding $\mathrm{p} 27-\mathrm{KID}^{\mathrm{wt}}$ and $\mathrm{p} 27-\mathrm{KID}^{+\mathrm{H}}\left(+32.16 \pm 1.22 \mathrm{kcal} \mathrm{mol}^{-1}\right.$ and $+22.3 \pm 2.46 \mathrm{kcal} \mathrm{mol}^{-1}$ respectively) to P-Cdk2/cyclin A seem to support this hypothesis, an analysis of the values of the other thermodynamic parameters does not. If the $\mathrm{p} 27-\mathrm{KID}^{+\mathrm{H}}$ bound to the P-Cdk2/cyclin A complex in the same way as the p27-KID wt, its association with the binary complex would be more favorable than that of the $\mathrm{p} 27-\mathrm{KID}^{\mathrm{wt}}$. However, from my experimental 
observation, this is not the case. The $\Delta G$ values of the association of $\mathrm{p} 27-\mathrm{KID}^{\mathrm{wt}}$ and $\mathrm{p} 27-\mathrm{KID}^{+\mathrm{H}}$ with P-Cdk2/cyclin A are $-11.61 \pm 0.3 \mathrm{kcal} \mathrm{mol}^{-1}$ and $-10.42 \pm 0.15$ $\mathrm{kcal} \mathrm{mol}^{-1}$, respectively (Table 4.1). The $\Delta \Delta \mathrm{G}$ value of the association of $\mathrm{p} 27-\mathrm{KID}^{+\mathrm{H}}$ and $\mathrm{P}-\mathrm{Cdk} 2 / \mathrm{cyclin} \mathrm{A}$ with respect to the $\mathrm{p} 27-\mathrm{KID}^{\mathrm{wt}}$ is $+1.2 \mathrm{kcal} \mathrm{mol}^{-1}$. An explanation for the less favorable free energy of association of $\mathrm{p} 27-\mathrm{KID}^{+\mathrm{H}}$ and $\mathrm{P}-\mathrm{Cdk} 2 /$ cyclin A with respect to $\mathrm{p} 27-\mathrm{KID}^{\mathrm{wt}}$ can be found in the values of the enthalpy that were observed for the two p27-KID variants. The enthalpies of association for the interaction of $\mathrm{p} 27-\mathrm{KID}^{\mathrm{wt}}$ and $\mathrm{p} 27-\mathrm{KID}^{+\mathrm{H}}$ with $\mathrm{P}-\mathrm{Cdk} 2 / \mathrm{cyclin} \mathrm{A}$ are $-43.77 \pm 1.38 \mathrm{kcal} \mathrm{mol}^{-1}$ and $-32.72 \pm 2.57 \mathrm{kcal} \mathrm{mol}^{-1}$. The heat released by the binding of $\mathrm{p} 27-\mathrm{KID}^{+\mathrm{H}}$ to $\mathrm{P}-\mathrm{Cdk} 2 / \mathrm{cyclin} \mathrm{A}$ is less than that for the $\mathrm{p} 27-\mathrm{KID}^{\mathrm{wt}}$. This indicates the p27-KID ${ }^{+H}$ mutant does not make exactly the same contacts as does $\mathrm{p} 27-\mathrm{KID}^{\mathrm{wt}}$ on binding P-Cdk2/cyclin A. Where are there differences in the contacts made by $\mathrm{p} 27-\mathrm{KID}^{+\mathrm{H}}$ and $\mathrm{p} 27-\mathrm{KID}^{\mathrm{wt}}$ on binding P-Cdk2/cyclin A?

It is unlikely the net enthalpy decrease in the binding of $\mathrm{p} 27-\mathrm{KID}^{+\mathrm{H}}$ to $\mathrm{P}-\mathrm{Cdk2} / \mathrm{cyclin} \mathrm{A}$ relative to $\mathrm{p} 27-\mathrm{KID}^{\mathrm{wt}}$ is due to lack of binding or proper folding of either D1 or D2. If D1 did not bind, the binding affinity of the interaction of p27-KID ${ }^{+H}$ and P-Cdk2/cyclin A $\left(K_{d}=23.41 \pm 6.09 \mathrm{nM}\right)$ should be similar to the binding affinity of the interactions of p27 to Cdk2 $(70.3 \pm 23.1 \mathrm{nM})^{197}$ or D2 peptide to $\mathrm{P}$-Cdk2/cyclin A $\left(K_{d}=82.96 \pm 17.89 \mathrm{nM}\right)$, which it does not. The results from the P-Cdk2/cyclin A kinase inhibition assays (Table 4.3) show that D2 binds and folds, like the p27-KID ${ }^{\mathrm{wt}}$, into an inhibition competent conformation. This indicates that the reduced enthalpy observed for the binding of $\mathrm{p} 27-\mathrm{KID}^{+\mathrm{H}}$ to $\mathrm{P}-\mathrm{Cdk} 2 / \mathrm{cyclin} \mathrm{A}$ relative to $\mathrm{p} 27-\mathrm{KID}^{\mathrm{wt}}$ stems from differences in the favorable 
contacts within the linker domain of $\mathrm{p} 27-\mathrm{KID}^{+\mathrm{H}}$. Since the linker domain of the p27- $\mathrm{KID}^{+\mathrm{H}}$ is already stabilized and does not need fold to the same extent as the p27-KID ${ }^{\text {wt }}$ on binding the P-Cdk2/cyclin A complex, the heat that is released during the folding of the linker domain helix of $\mathrm{p} 27-\mathrm{KID}^{+\mathrm{H}}$ on binding $\mathrm{P}-\mathrm{Cdk2/cyclin} \mathrm{A}$ is reduced.

In addition to the thermodynamic studies of the $\mathrm{p} 27-\mathrm{KID}^{+\mathrm{H}}$, the studies of the $\mathrm{p} 27-\mathrm{KID}^{-\mathrm{H}}$ also show that the nascent helix of $\mathrm{p} 27$ does not reduce the entropic cost associated with binding. The $-\mathrm{T} \Delta \mathrm{S}$ value of $\mathrm{p} 27-\mathrm{KID}^{-\mathrm{H}}$ and p27-KID ${ }^{\mathrm{wt}}$ on binding P-Cdk2/cyclin A are $+18.96 \pm 0.95 \mathrm{kcal} \mathrm{mol}^{-1}$ and $+32.16 \pm$ $1.22 \mathrm{kcal} \mathrm{mol}^{-1}$, respectively. . If the $\mathrm{p} 27-\mathrm{KID}^{-\mathrm{H}}$ mutant folds in the same way as p27-KID ${ }^{\text {wt }}$ upon binding, its entropic cost should be higher than that for wild-type p27-KID. This suggests that the $\mathrm{p} 27-\mathrm{KID}^{-\mathrm{H}}$ mutant does not fold to the same extent as p27-KID ${ }^{w t}$. The ability of $\mathrm{p} 27-\mathrm{KID}^{-\mathrm{H}}$ to inhibit $\mathrm{P}-\mathrm{Cdk} 2 / \mathrm{cyclin} \mathrm{A}$ kinase activity shows that the conformation of D2 on binding the kinase complex is similar in both the $\mathrm{p} 27-\mathrm{KID}^{\mathrm{wt}}$ and $\mathrm{p} 27-\mathrm{KID}^{-\mathrm{H}}$. The $\mathrm{IC}_{50}$ values for $\mathrm{p} 27-\mathrm{KID}^{\mathrm{wt}}$ and $\mathrm{p} 27-\mathrm{KID}^{-\mathrm{H}}$ are $0.3 \mathrm{nM}$ and $0.35 \mathrm{nM}$ respectively (Table 4.3). It is unlikely that $\mathrm{D} 1$ in the $\mathrm{p} 27-\mathrm{KID}^{-\mathrm{H}}$ mutant is not binding since the dissociation constant of the binding of the D2 peptide to P-Cdk2/cyclin A $(82.96 \pm 17.89 \mathrm{nM})$ is much higher than that observed for the $\mathrm{p} 27-\mathrm{KID}^{-\mathrm{H}}(3.66 \pm 0.91 \mathrm{nM})$. This implies that the linker domain of this mutant does not fold on binding P-Cdk2/cyclin A. This is the most likely cause of the decreased enthalpy of binding observed for the association of the $\mathrm{p} 27-\mathrm{KID}^{-\mathrm{H}}$ and the $\mathrm{P}-\mathrm{Cdk} 2 / \mathrm{cyclin} \mathrm{A}$ relative to $\mathrm{p} 27-\mathrm{KID}^{\mathrm{wt}}$. The observed enthalpies for the binding of the $\mathrm{p} 27-\mathrm{KID}^{\mathrm{wt}}$ and $\mathrm{p} 27-\mathrm{KID}^{-\mathrm{H}}$ with the 
$\mathrm{P}-\mathrm{Cdk} 2 /$ cyclin A are $-43.77 \pm 1.38 \mathrm{kcal} \mathrm{mol}^{-1}$ and $-30.48 \pm 0.82$ respectively.

Like the $\mathrm{p} 27-\mathrm{KID}^{+\mathrm{H}}$ and $\mathrm{p} 27-\mathrm{KID}^{-\mathrm{H}}$ mutants, the ITC thermodynamic parameters and the P-Cdk2/cyclin A kinase inhibition assays indicate that the D1 and D2 domains of the p27-KID ${ }^{\text {loop }}$ mutant bind to P-Cdk2/cyclin A. The binding of the D2 domain of the p27-KID ${ }^{\text {loop }}$ mutant is shown by its ability to potently and completely inhibit P-Cdk2/cyclin A kinase activity $\left(\mathrm{IC}_{50}=16 \mathrm{nM}\right)$. In Section 5.1 above, I discussed that complete kinase inhibition requires coupling of D1 and D2 by the linker domain. The ability of the $\mathrm{p} 27-\mathrm{KID}^{\mathrm{loop}}$ mutant to completely inhibit kinase activity indicates that the D1 domain of this mutant binds to the cyclin A subunit of $\mathrm{P}$-Cdk2/cyclin A. The $-\mathrm{T} \Delta \mathrm{S}$ value of this mutant $(+19.7 \pm 1.36$ $\mathrm{kcal} \mathrm{mol}^{-1}$ ) therefore shows that its linker domain does not fold to the same extent as the $\mathrm{p} 27-\mathrm{KID}^{\mathrm{wt}}\left(-\mathrm{T} \Delta \mathrm{S}=+32.16 \pm 1.22 \mathrm{kcal} \mathrm{mol}^{-1}\right)$ on on binding $\mathrm{P}-\mathrm{Cdk} 2 / \mathrm{cyclin}$ A. This is not surprising since this mutant was designed with residues that would minimize the formation of any helical structure. Expectedly, the enthalpy of the p27-KID ${ }^{\text {loop }}$ mutant on binding P-Cdk2/cyclin A is less than the enthalpy of the interaction $\mathrm{p} 27-\mathrm{KID}^{\mathrm{wt}}$ and the same complex. The enthalpies of the interactions of p27-KID loop and p27-KID wt with P-Cdk2/cyclin A are $-29.12 \pm 1.19 \mathrm{kcal} \mathrm{mol}^{-1}$ and $-43.77 \pm 1.38 \mathrm{kcal} \mathrm{mol}^{-1}$, respectively. The results in the studies above suggest that the folding of the linker domain of the wild-type p27 may be an inherent feature of its sequence and is possibly the reason the for the conservation of the p27 linker domain sequence in different species.

Stabilization of the linker domain affects the binding affinity of p27 for 
P-Cdk2/cyclin A. The $K_{d}$ values for the $\mathrm{p} 27-\mathrm{KID}^{\mathrm{wt}}$ and $\mathrm{p} 27-\mathrm{KID}^{+\mathrm{H}}$ were determined to be $3.34 \pm 1.64 \mathrm{nM}$ and $23.41 \pm 6.09 \mathrm{nM}$ respectively. The dissociation constants of the binding of the $\mathrm{p} 27-\mathrm{KID}^{-\mathrm{H}}$ and $\mathrm{p} 27-\mathrm{KID}^{\mathrm{loop}}$ mutants to the P-Cdk2/cyclin A complex were $3.66 \pm 0.91 \mathrm{nM}$ and $138.3 \pm 43.05 \mathrm{nM}$ respectively. The $\mathrm{p} 27-\mathrm{KID}^{-\mathrm{H}}$ mutant has the same affinity for the $\mathrm{P}-\mathrm{Cdk} 2 / \mathrm{cyclin} \mathrm{A}$ complex as the wild-type p27-KID, suggesting that flexibility of the linker domain may be important for binding to the P-Cdk2/cyclin A complex. On the other hand, restriction of the motions of the linker domain $\left(\mathrm{p} 27-\mathrm{KID}^{+\mathrm{H}}\right)$ decreases the affinity of p27 for the P-Cdk2/cyclin A complex. However, complete elimination of the helical structure of the linker domain (p27-KID ${ }^{\text {loop }}$ ) greatly decreases affinity for the P-Cdk2/cyclin A complex. Together these data suggest that the dynamics of the linker domain are necessary for tight binding of p27 to P-Cdk2/cyclin A.

The thermal denaturation results indicate that stabilization or destabilization of the helical structure of the linker domain of p27 decreases the thermal stability of the ternary complex formed when p27 binds P-Cdk2/cyclin A. Melting temperatures for the ternary complexes of the p27-KID ${ }^{w t}, \mathrm{p} 27-\mathrm{KID}^{+\mathrm{H}}$, p27-KID ${ }^{-H}$, and p27-KID ${ }^{\text {loop }}$ were $82.3 \pm 0.5^{\circ} \mathrm{C}, 80.3 \pm 0.2^{\circ} \mathrm{C}, 78.5 \pm 0.6{ }^{\circ} \mathrm{C}$, and $73.0 \pm 0.3{ }^{\circ} \mathrm{C}$ respectively. The thermal stability of the ternary complexes is determined by the difference between the free energies of the native and unfolded states of these complexes. The more negative the difference obtained by subtracting the free energy of the native state from the free energy of the unfolded state, the more unstable the complex. The destabilization of the ternary complexes $\mathrm{p} 27-\mathrm{KID}^{+\mathrm{H}}$ and $\mathrm{p} 27-\mathrm{KID}^{\mathrm{loop}}$ mutants relative to that of $\mathrm{p} 27-\mathrm{KID}^{\mathrm{wt}}$ is 
therefore expected since the free energies of their native states are less favorable relative to that of the $\mathrm{p} 27-\mathrm{KID}^{\mathrm{wt}}$. The Gibbs free energy values of the native states of the $\mathrm{p} 27-\mathrm{KID}^{\mathrm{wt}}, \mathrm{p} 27-\mathrm{KID}^{+\mathrm{H}}$, and $\mathrm{p} 27-\mathrm{KID}^{\text {loop }}$ are $-11.61 \pm 0.30 \mathrm{kcal}$ $\mathrm{mol}^{-1},-10.42 \pm 0.15 \mathrm{kcal} \mathrm{mol}^{-1}$, and $-9.37 \pm 0.18 \mathrm{kcal} \mathrm{mol}^{-1}$, respectively (Table 4.1). On the other hand, the free energy of the native state of the ternary complex of the p27-KID ${ }^{-H}$ mutant $\left(\Delta G=-11.52 \pm 0.14 \mathrm{kcal} \mathrm{mol}^{-1}\right)$ has the same free energy as that for $\mathrm{p} 27-\mathrm{KID}^{\mathrm{wt}}\left(-11.61 \pm 0.30 \mathrm{kcal} \mathrm{mol}^{-1}\right)$ within standard error. The destabilization of this mutant therefore stems from the stabilization of its unfolded state. The destabilization of these mutants with structurally perturbed linker domains suggests that the structure of the wild-type linker domain of p27 is important for formation of a stable ternary complex.

Although p27-KID ${ }^{\mathrm{wt}}$ is as potent as $\mathrm{p} 27-\mathrm{KID}^{-\mathrm{H}}$ in inhibiting P-Cdk2/cyclin A kinase activity, it is more potent than either $\mathrm{p} 27-\mathrm{KID}^{+\mathrm{H}}$ or $\mathrm{p} 27-\mathrm{KID}^{\text {loop }}$. The observed $\mathrm{IC}_{50}$ values for the p27-KID ${ }^{\mathrm{wt}}$, p27-KID ${ }^{-\mathrm{H}}, \mathrm{p} 27-\mathrm{KID}^{+\mathrm{H}}$ and $\mathrm{p} 27-\mathrm{KID}^{\text {loop }}$ were $0.3 \mathrm{nM}, 0.35 \mathrm{nM}, 5 \mathrm{nM}$ and $16 \mathrm{nM}$ respectively. Being potent kinase inhibitors, I expected these mutants to be effective in causing cell cycle arrest. However, cell cycle arrest analysis showed that all of the p27 mutants do not cause cell cycle arrest. When flp2 $27^{\mathrm{wt}}$, flp2 $27^{+\mathrm{H}}$, flp2 $7^{-\mathrm{H}}$, and flp2 $7^{\text {loop }}$ are over-expressed in mouse fibroblasts, $14 \%, 18.2 \%, 28.1 \%$, and $30.5 \%$ of the cells progressed to the S phase relative to a vector only control in which $28.4 \%$ of the cells progress to $S$ phase (Table 4.4). These results show that while stabilizing the linker domain of p27 in cells impairs the cell cycle arrest function, destabilizaing the linker domain or completely eliminating its helical structure 
completely annuls the capacity of $p 27$ to cause cell cycle arrest. The raises a question as to why the flp $27^{-\mathrm{H}}$ and flp $27^{\mathrm{loop}}$ mutants cannot cause cell cycle arrest even though they are potent inhibitors of P-Cdk2/cyclin A kinase activity in vitro. One explanation is that the structural changes in the linker domains of these mutants may alter their specificity. During G1/S cell cycle transition, Cdk4, Cdk6, Cdk1, and Cdk2 kinase complexes phosphorylate a number of downstream targets facilitating the progression of the cell cycle from the $\mathrm{G} 1$ to the S phase. The altered specificity of these mutants may decrease their binding to one or more of these Cdk complexes resulting in leaky inhibition of the G1/S transition. This may explain the results observed for the flp2 $7^{-\mathrm{H}}$ and flp2 $7^{\text {loop }}$ mutants. The results of the cell cycle arrest analysis studies attest to the significance of the structure of the linker domain of p27 in biological function. 


\section{List of References}

1 Cooper GM. The Cell: a molecular approach. ASM Press: Washington, DC, 2000.

2 Lodish HF et al. Molecular Cell Biology. W. H. Freeman: New York, NY, 2000.

$3 \quad$ Elledge SJ et al. CDK2 encodes a 33-kDa cyclin A-associated protein kinase and is expressed before CDC2 in the cell cycle. Proc Natl Acad Sci U S A 1992; 89: 2907-2911.

$4 \quad$ Malumbres M, Barbacid M. Mammalian cyclin-dependent kinases. Trends Biochem Sci 2005; 30: 630-641.

5 Meyerson M, Harlow E. Identification of G1 kinase activity for cdk6, a novel cyclin D partner. Mol Cell Biol 1994; 14: 2077-2086.

6 Rosenblatt J, Gu Y, Morgan DO. Human cyclin-dependent kinase 2 is activated during the $S$ and $G 2$ phases of the cell cycle and associates with cyclin A. Proc Natl Acad Sci U S A 1992; 89: 2824-2828.

7 Doree M, Galas S. The cyclin-dependent protein kinases and the control of cell division. Faseb J 1994; 8: 1114-1121.

8 Boxem M. Cyclin-dependent kinases in C. elegans. Cell Div 2006; 1: 6.

$9 \quad$ Fisher RP. CDKs and cyclins in transition(s). Curr Opin Genet Dev 1997; 7: 32-38.

10 Grana X, Reddy EP. Cell cycle control in mammalian cells: role of cyclins, cyclin dependent kinases (CDKs), growth suppressor genes and cyclindependent kinase inhibitors (CKIs). Oncogene 1995; 11: 211-219.

11 Sanchez I, Dynlacht BD. New insights into cyclins, CDKs, and cell cycle control. Semin Cell Dev Biol 2005; 16: 311-321.

12 Morgan DO. Principles of CDK regulation. Nature 1995; 374: 131-134.

13 Koff $A$ et al. Human cyclin $E$, a new cyclin that interacts with two members of the CDC2 gene family. Cell 1991; 66: 1217-1228.

14 Draetta GF. Mammalian G1 cyclins. Curr Opin Cell Biol 1994; 6: 842-846.

15 Sherr CJ. Mammalian G1 cyclins and cell cycle progression. Proc Assoc Am Physicians 1995; 107: 181-186. 
16 Roberts JM et al. Cyclins, Cdks, and cyclin kinase inhibitors. Cold Spring Harbor Symposia on Quantitative Biology 1994: 31-38.

17 Ekholm SV, Reed SI. Regulation of G(1) cyclin-dependent kinases in the mammalian cell cycle. Curr Opin Cell Biol 2000; 12: 676-684.

18 Stewart E, Kobayashi H, Harrison D, Hunt T. Destruction of Xenopus cyclins $\mathrm{A}$ and $\mathrm{B} 2$, but not $\mathrm{B} 1$, required binding to $\mathrm{p} 34 \mathrm{cdc}$. The EMBO J 1994; 13: 584-594.

19 Johnson DG, Walker CL. Cyclins and cell cycle checkpoints. Annu Rev Pharmacol Toxicol 1999; 39: 295-312.

20 Solomon MJ. The function(s) of CAK, the p34cdc2-activating kinase. Trends Biochem Sci 1994; 19: 496-499.

21 Liu Y, Wu C, Galaktionov K. p42, a novel cyclin-dependent kinaseactivating kinase in mammalian cells. J Biol Chem 2004; 279: 4507-4514.

22 Kaldis P, Solomon MJ. Analysis of CAK activities from human cells. Eur $J$ Biochem 2000; 267: 4213-4221.

23 Kaldis $\mathrm{P}$. The cdk-activating kinase (CAK): from yeast to mammals. Cell Mol Life Sci 1999; 55: 284-296.

$24 \quad$ Kaldis $\mathrm{P}$ et al. Human and yeast cdk-activating kinases (CAKs) display distinct substrate specificities. Mol Biol Cell 1998; 9: 2545-2560.

25 Umeda $\mathrm{M}$ et al. A distinct cyclin-dependent kinase-activating kinase of Arabidopsis thaliana. Proc Natl Acad Sci U S A 1998; 95: 5021-5026.

26 Buck V, Russell P, Millar JB. Identification of a cdk-activating kinase in fission yeast. The EMBO J 1995; 14: 6173-6183.

27 Brown NR et al. Effects of phosphorylation of threonine 160 on cyclindependent kinase 2 structure and activity. J Biol Chem 1999; 274: 87468756.

28 Mittnacht S. Control of pRB phosphorylation. Curr Opin Genet Dev 1998; 8: 21-27.

29 Berthet $\mathrm{C}$ et al. Combined loss of Cdk2 and Cdk4 results in embryonic lethality and Rb hypophosphorylation. Dev Cell 2006; 10: 563-573.

30 Akiyama $T$ et al. Phosphorylation of the retinoblastoma protein by cdk2. Proc Natl Acad Sci U S A 1992; 89: 7900-7904. 
31 Takaki T et al. Preferences for phosphorylation sites in the retinoblastoma protein of D-type cyclin-dependent kinases, Cdk4 and Cdk6, in vitro. $J$ Biochem (Tokyo) 2005; 137: 381-386.

32 Suzuki-Takahashi I et al. The interactions of E2F with pRB and with p107 are regulated via the phosphorylation of pRB and p107 by a cyclindepednent kinase. Oncogene 1995; 10: 1691-1698.

33 Dyson N. The regulation of E2F by pRB-family proteins. Genes Dev 1998; 12: $2245-2262$.

34 Frolov MV, Dyson NJ. Molecular mechanisms of E2F-dependent activation and pRB-mediated repression. J Cell Sci 2004; 117: 2173-2181.

35 Tyers M, Jorgensen P. Proteolysis and the cell cycle: with this RING I do thee destroy. Curr Opin Genet Dev 2000; 10: 54-64.

36 Reed SI. Control of the G1/S transition. Cancer Surv 1997; 29: 7-23.

37 Sherr CJ. Mammalian G1 cyclins. Cell 1993; 73: 1059-1065.

38 Krek W. Proteolysis and the G1-S transition: the SCF connection. Curr Opin Genet Dev 1998; 8: 36-42.

39 Nurse $P$, Thuriaux $P$. Regulatory genes controlling mitosis in the fission yeast Schizosaccharomyces pombe. Genetics 1980; 96: 627-637.

$40 \quad$ Nurse P. Universal control mechanism regulating onset of M-phase. Nature 1990; 344: 503-508.

41 Nasmyth K. Control of the yeast cell cycle by the Cdc28 protein kinase. Curr Opin Cell Biol 1993; 5: 166-179.

42 Elledge SJ, Spottswood MR. A new human p34 protein kinase, CDK2, identified by complementation of a cdc28 mutation in Saccharomyces cerevisiae, is a homolog of Xenopus Eg1. The EMBO J 1991; 10: 26532659.

43 Harper JW, Elledge SJ. The role of Cdk7 in CAK function, a retroretrospective. Genes Dev 1998; 12: 285-289.

44 Fisher RP, Morgan DO. A novel cyclin associates with MO15/CDK7 to form the CDK-activating kinase. Cell 1994; 78: 713-724.

45 Dhavan R, Tsai LH. A decade of CDK5. Nat Rev Mol Cell Biol 2001; 2: 749-759. 
46 Gupta A, Tsai LH. Cyclin-dependent kinase 5 and neuronal migration in the neocortex. Neurosignals 2003; 12: 173-179.

47 Akoulitchev S, Chuikov S, Reinberg D. TFIIH is negatively regulated by cdk8-containing mediator complexes. Nature 2000; 407: 102-106.

48 Furumoto $T$ et al. A kinase subunit of the human mediator complex, CDK8, positively regulates transcriptional activation. Genes Cells 2007; 12: 119-132.

49 Najello B, Napolitano G, Giordane A, Lania L. Transcriptional regulation by targerted recruitment of cyclin-dependent CDK9 kinase in vivo. Oncogene 1999; 10: 4598-4605.

50 Baek K, Brown RS, Birrane G, Ladias JA. Crystal structure of human cyclin K, a positive regulator of cyclin-dependent Kinase 9. J Mol Biol 2007; 366: 563-573.

51 De Bondt $\mathrm{HL}$ et al. Crystal structure of cyclin-dependent kinase 2. Nature 1993; 363: 595-602.

52 Jeffrey PD et al. Mechanism of CDK activation revealed by the structure of a cyclinA-CDK2 complex. Nature 1995; 376: 313-320.

53 Honda R et al. The structure of cyclin E1/CDK2: implications for CDK2 activation and CDK2-independent roles. The EMBO J 2005; 24: 452-463.

54 Schulman BA, Lindstrom DL, Harlow E. Substrate recruitment to cyclindependent kinase 2 by a multipurpose docking site on cyclin A. Proc Natl Acad Sci U S A 1998; 95: 10453-10458.

55 Terada $\mathrm{Y}$, Tatsuka M, Jinno S, Okayama H. Requirement for tyrosine phosphorylation of Cdk4 in G1 arrest induced by ultraviolet irradiation. Nature 1995; 376: 358-362.

56 lavarone A, Massague J. Repression of the CDK activator Cdc25A and cell-cycle arrest by cytokine TGF-beta in cells lacking the CDK inhibitor p15. Nature 1997; 387: 417-422.

57 Chen S, Gardner DG. Suppression of WEE1 and stimulation of CDC25A correlates with endothelin-dependent proliferation of rat aortic smooth muscle cells. J Biol Chem 2004; 279: 13755-13763.

58 Gu Y, Rosenblatt J, Morgan DO. Cell cycle regulation of CDK2 activity by phosphorylation of Thr160 and Tyr15. The EMBO J 1992; 11: 3995-4005. 
59 Chow JP et al. Differential contribution of inhibitory phosphorylation of CDC2 and CDK2 for unperturbed cell cycle control and DNA integrity checkpoints. J Biol Chem 2003; 278: 40815-40828.

60 McGowan $\mathrm{CH}$, Russell P. Human Wee1 kinase inhibits cell division by phosphorylating p34cdc2 exclusively on Tyr15. The EMBO J 1993; 12: 7585.

61 Mueller PR, Coleman TR, Kumagai A, Dunphy WG. Myt1: a membraneassociated inhibitory kinase that phosphorylates Cdc2 on both threonine14 and tyrosine-15. Science 1995; 270: 86-90.

62 Fattaey A, Booher RN. Myt1: a Wee1-type kinase that phosphorylates Cdc2 on residue Thr14. Prog Cell Cycle Res 1997; 3: 233-240.

63 Touny LH, Banerjee PP. Identification of both Myt-1 and Wee-1 as necessary mediators of the p21-independent inactivation of the cdc2/cyclin B1 complex and growth inhibition of TRAMP cancer cells by genistein. Prostate 2006; 66: 1542-1555.

64 Squire CJ, Dickson JM, Ivanovic I, Baker EN. Structure and inhibition of the human cell cycle checkpoint kinase, Wee1A kinase: an atypical tyrosine kinase with a key role in CDK1 regulation. Structure 2005; 13: 541-550.

65 Sadhu K, Reed SI, Richardson H, Russell P. Human homolog of fission yeast cdc25 mitotic inducer is predominantly expressed in G2. Proc Natl Acad Sci U S A 1990; 87: 5139-5143.

66 Millar JB, Russell P. The cdc25 M-phase inducer: an unconventional protein phosphatase. Cell 1992; 68: 407-410.

67 Millar J et al. cdc25 M-phase inducer. Cold Spring Harb Symp Quant Biol 1991; 56: 577-584.

68 De Clercq A, Inze D. Cyclin-dependent kinase inhibitors in yeast, animals, and plants: a functional comparison. Crit Rev Biochem Mol Biol 2006; 41: 293-313.

69 Harper JW. Cyclin dependent kinase inhibitors. Cancer Surv 1997; 29: $91-$ 107.

70 Hannon GJ, Beach D. p15ink4b is a potential effector of Tgf-(-induced cell cycle arrest. Nature 1994; 371: 257-261.

71 Raus M, Peters $\mathrm{G}$. The p16INK4A/CDKN2A tumor suppressor and its relatives. Biochim Biophys Acta Rev Cancer 1998; 1378: F115-F177. 
$72 \quad$ Hirai $\mathrm{H}$ et al. Novel INK4 proteins, $\mathrm{p} 19$ and p18, are specific inhibitors of the cyclin D-dependent kinases CDK4 and CDK6. Mol Cell Biol 1995; 15: 2672-2681.

73 Chan FK et al. Identification of human and mouse p19, a novel CDK4 and CDK6 inhibitor with homology to p16ink4. Mol Cell Biol 1995; 15: 26822688.

74 Harper JW et al. The p21 Cdk-interacting protein Cip1 is a potent inhibitor of G1 cyclin-dependent kinases. Cell 1993; 75: 805-816.

75 Adkins JN, Lumb KJ. Stoichiometry of cylin A-cyclin dependent kinase 2 inhibition by p21 ${ }^{\text {Cip1/Waf1 }}$. Biochemistry 2000; 39: 13925-13930.

76 Chan J, Jackson PK, Kirschner MW, Dutta A. Separate domains of p21 involved in the inhibition of Cdk kinase and PCNS. Nature 1995; 374: 386388.

77 Fotedar $\mathrm{G}$ et al. p21 contains independent binding sites for cyclin and cdk2: both sites are required to inhibit cdk2 kinase activity. Oncogene 1996; 12: 2155-2289.

$78 \quad$ Xiong $\mathrm{Y}$ et al. p21 is a universal inhibitor of cyclin kinases. Nature 1993; 366: 701-704.

79 Huppi K et al. Molecular cloning, sequencing, chromosomal localization and expression of mouse p21 (Waf1). Oncogene 1994; 9: 3017-3020.

80 Polyak K et al. p27kip1, a cyclin-Cdk inhibitor, links transforming growth factor-beta and contact inhibition to cell cycle arrest. Genes Dev 1994; 8: 9-22.

81 Polyak K et al. Cloning of p27Kip1, a cyclin-dependent kinase inhibitor and a potential mediator of extracellular antimitogenic signals. Cell 1994; 78: 59-66.

82 Ponce-Castañeda $V$ et al. p27kip1: Chromosomal mapping to 12p12-1213.1 and absence of mutations in human tumors. Cancer Research 1995; 55: 1211-1214.

83 Toyoshima $\mathrm{H}$, Hunter T. p27, a novel inhibitor of $\mathrm{G} 1$ cyclin-Cdk protein kinase activity, is related to p21. Cell 1994; 78: 67-74.

84 Ohnuma Si et al. p27xic1, a Cdk inhibitor, promotes the determination of glial cells in Xenopus retina. Cell 1999; 99: 499-510. 
85 Nomura H, Sawada Y, Fujinaga K, Ohtaki S. Cloning and characterization of rat p27Kip1, a cyclin-dependent kinase inhibitor. Gene 1997; 191: 211218.

86 Matsuoka S et al. p57KIP2, a structurally distinct member of the p21CIP1 Cdk inhibitor family, is a candidate tumor suppressor gene. Genes Dev 1995; 9: 650-652.

87 Lee MH, Reynisdóttir I, Massagué J. Cloning of p57KIP2, a cyclindependent kinase inhibitor with unique domain structure and tissue distribution. Genes Dev 1995; 9: 639-649.

$88 \quad$ Nakano $\mathrm{N}$ et al. Downregulation of cyclin-dependent kinase inhibitor; p57(kip2), is involved in the cell cycle progression of vascular smooth muscle cells. Biochem Biophys Res Commun 2005; 338: 1661-1667. Epub 2005 Oct 1625.

89 Russo AA et al. Structural basis for inhibition of the cyclin-dependent kinase Cdk6 by the tumour suppressor p16INK4a. Nature 1998; 395: 237243.

90 Knighton DR et al. Crystal structure of the catalytic subunit of cyclic adenosine monophosphate-dependent protein kinase. Science 1991; 253: 407-414.

91 Nakayama Ki, Nakayama K. Cip/Kip cyclin-dependent kinase inhibitors: brakes of the cell cycle engine during development. BioEssays 1998; 20: 1020-1029.

92 Hengst L, Reed SI. Inhibitors of the Cip/Kip family. Curr Top Microbiol Immunol 1998; 227: 25-41.

93 Cheng $\mathrm{M}$ et al. The p21(Cip1) and p27(Kip1) CDK 'inhibitors' are essential activators of cyclin D-dependent kinases in murine fibroblasts. The EMBO J 1999; 18: 1571-1583.

94 Sherr CJ, Roberts JM. Cdk inhibitors: positive and negative regulators of G1-phase progression. Genes Dev 1999; 13: 1501-1512.

95 Sugimoto $\mathrm{M}$ et al. Activation of cyclin D1-kinase in murine fibroblasts lacking both p21(Cip1) and p27(Kip1). Oncogene 2002; 21: 8067-8074.

96 Bagui TK, Mohapatra S, Haura E, Pledger WJ. P27Kip1 and p21Cip1 are not required for the formation of active D cyclin-cdk4 complexes. Mol Cell Biol 2003; 23: 7285-7290.

$97 \quad$ Grimmler $\mathrm{M}$ et al. Cdk-inhibitory activity and stability of p27Kip1 are directly regulated by oncogenic tyrosine kinases. Cell 2007; 128: 269-280. 
98 el-Deiry WS et al. WAF1/CIP1 is induced in p53-mediated G1 arrest and apoptosis. Cancer Res 1994; 54: 1169-1174.

99 Di Leonardo A, Linke SP, Clarkin K, Wahl GM. DNA damage triggers a prolonged p53-dependent $\mathrm{G} 1$ arrest and long-term induction of Cip1 in normal human fibroblasts. Genes Dev 1994; 8: 2540-2551.

100 Qian X, Jin L, Grande JP, Lloyd RV. Transforming growth factor-beta and p27 expression in pituitary cells. Endocrinology 1996; 137: 3051-3060.

101 Nourse $\mathrm{J}$ et al. Interleukin-2-mediated elimination of the p27Kip1 cyclindependent kinase inhibitor prevented by rapamycin. Nature 1994; 372: 570-573.

102 Poon RY, Toyoshima H, Hunter T. Redistribution of the CDK inhibitor p27 between different cyclin.CDK complexes in the mouse fibroblast cell cycle and in cells arrested with lovastatin or ultraviolet irradiation. Mol Biol Cell 1995; 6: 1197-1213.

103 Fang $\mathrm{F}$ et al. Dependence of cyclin E-CDK2 kinase activity on cell anchorage. Science 1996; 271: 499-502.

104 Russo AA et al. Crystal structure of the p27Kip1 cyclin-dependent-kinase inhibitor bound to the cyclin A-Cdk2 complex. Nature 1996; 382: 325-331.

105 Durand B, Gao FB, Raff M. Accumulation of the cyclin-dependent kinase inhibitor p27/Kip1 and the timing of oligodendrocyte differentiation. The $E M B O$ J 1997; 16: 306-317.

106 Tikoo R, Casaccia-Bonnefil P, Chao MV, Koff A. Changes in cyclindependent kinase 2 and p27kip1 accompany glial cell differentiation of central glia-4 cells. J Biol Chem 1997; 272: 442-447.

107 Besson A et al. p27Kip1 modulates cell migration through the regulation of RhoA activation. Genes Dev 2004; 18: 862-876. Epub 2004 Apr 2012.

108 Rivard N, L'Allemain G, Bartek J, Pouyssegur J. Abrogation of p27Kip1 by cDNA antisense suppresses quiescence (G0 state) in fibroblasts. $J$ Biol Chem 1996; 271: 18337-18341.

109 Agrawal D et al. Repression of p27kip1 synthesis by platelet-derived growth factor in BALB/c 3T3 cells. Mol Cell Biol 1996; 16: 4327-4336.

110 Kato JY et al. Cyclic AMP-induced G1 phase arrest mediated by an inhibitor (p27Kip1) of cyclin-dependent kinase 4 activation. Cell 1994; 79: 487-496. 
111 Gardner LB et al. Hypoxia inhibits G1/S transition through regulation of p27 expression. J Biol Chem 2001; 276: 7919-7926.

112 Olashaw N, Pledger WJ. Paradigms of growth control: relation to Cdk activation. Sci STKE 2002; 2002: RE7.

113 Nakayama K et al. Mice lacking p27(Kip1) display increased body size, multiple organ hyperplasia, retinal dysplasia, and pituitary tumors. Cell 1996; 85: 707-720.

114 Kiyokawa $\mathrm{H}$ et al. Enhanced growth of mice lacking the cyclin-dependent kinase inhibitor function of p27(Kip1). Cell 1996; 85: 721-732.

115 Fero ML et al. A syndrome of multiorgan hyperplasia with features of gigantism, tumorigenesis, and female sterility in p27Kip1-deficient mice. Cell 1996; 85: 733-744.

116 Fero $\mathrm{ML}$ et al. A syndrome of multiorgan hyperplasia with features of gigantism, tumorigenesis, and female sterility in p27(Kip1)-deficient mice. Cell 1996; 85: 733-744.

117 Eymin B, Brambilla E. The yin and the yang of p27Kip1 as a target for cancer therapy. Eur Respir J 2004; 23: 663-664.

118 Lloyd RV et al. p27kip1: a multifunctional cyclin-dependent kinase inhibitor with prognostic significance in human cancers. Am J Pathol 1999; 154: 313-323.

119 Pellegata NS et al. Germ-line mutations in p27Kip1 cause a multiple endocrine neoplasia syndrome in rats and humans. Proc Natl Acad Sci U S A 2006; 103: 15558-15563.

120 Morosetti $\mathrm{R}$ et al. Alterations of the p27KIP1 gene in non-Hodgkin's lymphomas and adult T-cell leukemia/lymphoma. Blood 1995; 86: 19241930.

121 Tanaka $\mathrm{C}$ et al. Infrequent mutations of p27Kip1 gene and trisomy 12 in a subset of human pituitary adenomas. J Clin Endocrinol Metab 1997; 82: 3141-3147.

122 Cariou S, Catzavelos C, Slingerland JM. Prognostic implications of expression of the cell cycle inhibitor protein p27Kip1. Breast Cancer Res Treat 1998; 52: 29-41.

123 Tsuchiya A, Zhang GJ, Kanno M. Prognostic impact of cyclin-dependent kinase inhibitor p27kip1 in node-positive breast cancer. J Surg Oncol 1999; 70: 230-234. 
124 Chiarle R, Pagano M, Inghirami G. The cyclin dependent kinase inhibitor p27 and its prognostic role in breast cancer. Breast Cancer Res 2001; 3: 91-94.

125 Palmqvist R, Stenling R, Oberg A, Landberg G. Prognostic significance of p27(Kip1) expression in colorectal cancer: a clinico-pathological characterization. J Pathol 1999; 188: 18-23.

126 Catzavelos $\mathrm{C}$ et al. Reduced expression of the cell cycle inhibitor p27Kip1 in non-small cell lung carcinoma: a prognostic factor independent of Ras. Cancer Res 1999; 59: 684-688.

127 Chetty R. p27 Protein and cancers of the gastrointestinal tract and liver: an overview. J Clin Gastroenterol 2003; 37: 23-27.

128 Yang RM et al. Low p27 expression predicts poor disease-free survival in patients with prostate cancer. J Urol 1998; 159: 941-945.

129 Venkatesan TK et al. Prognostic significance of p27 expression in carcinoma of the oral cavity and oropharynx. Laryngoscope 1999; 109: 1329-1333.

130 Masciullo $\mathrm{V}$ et al. Frequent loss of expression of the cyclin-dependent kinase inhibitor p27 in epithelial ovarian cancer. Cancer Res 1999; 59: 3790-3794.

131 Zhang $Q$ et al. Inducible expression of a degradation-resistant form of p27Kip1 causes growth arrest and apoptosis in breast cancer cells. FEBS Lett 2005; 579: 3932-3940.

132 Koff A. How to decrease p27Kip1 levels during tumor development. Cancer Cell 2006; 9: 75-76.

133 Andreu EJ et al. BCR-ABL induces the expression of Skp2 through the PI3K pathway to promote p27Kip1 degradation and proliferation of chronic myelogenous leukemia cells. Cancer Res 2005; 65: 3264-3272.

134 Blain SW, Massague J. Breast cancer banishes p27 from nucleus. Nat Med 2002; 8: 1076-1078.

135 Kamura $\mathrm{T}$ et al. Cytoplasmic ubiquitin ligase KPC regulates proteolysis of p27(Kip1) at G1 phase. Nat Cell Biol 2004; 6: 1229-1235.

136 Sutterluty $\mathrm{H}$ et al. p45SKP2 promotes p27Kip1 degradation and induces S phase in quiescent cells. Nat Cell Biol 1999; 1: 207-214. 
137 Carrano AC, Eytan E, Hershko A, Pagano M. SKP2 is required for ubiquitin-mediated degradation of the CDK inhibitor p27. Nat Cell Biol 1999; 1: 193-199.

138 Tsvetkov LM et al. p27(Kip1) ubiquitination and degradation is regulated by the SCF(Skp2) complex through phosphorylated Thr187 in p27. Curr Biol 1999; 9: 661-664.

139 Pagano $\mathrm{M}$ et al. Role of the ubiquitin-proteasome pathway in regulating abundance of the cyclin-dependent kinase inhibitor p27. Science 1995; 269: 682-685.

140 Bloom J et al. Deregulated degradation of the cdk inhibitor p27 and malignant transformation ATM-dependent phosphorylation and accumulation of endogenous BLM protein in response to ionizing radiation; A comparison of cell cycle markers in well-differentiated lobular and ductal carcinomas MNNG-transformed Bloom syndrome Blymphoblastoids for the detection of Hodgkin's lymphoma-associated antigen in 2D Westerns. Semin Cancer Biol 2003; 13: 41-47.

141 Zhu XH et al. Noncatalytic requirement for cyclin A-cdk2 in p27 turnover. Mol Cell Biol 2004; 24: 6058-6066.

142 Keikhaee MR et al. Skp2 expression is associated with down-regulation of p27 protein and cell proliferation in salivary adenoid cystic carcinoma. Virchows Arch 2007.

143 Kitajima $S$ et al. Role of Cks1 overexpression in oral squamous cell carcinomas: cooperation with Skp2 in promoting p27 degradation. Am J Pathol 2004; 165: 2147-2155.

144 Kudo $Y$ et al. Small interfering RNA targeting of S phase kinase-interacting protein 2 inhibits cell growth of oral cancer cells by inhibiting p27 degradation. Mol Cancer Ther 2005; 4: 471-476.

145 Osoegawa A et al. Regulation of p27 by S-phase kinase-associated protein 2 is associated with aggressiveness in non-small-cell lung cancer. J Clin Oncol 2004; 22: 4165-4173.

146 Brown I et al. Reduced expression of p27 is a novel mechanism of docetaxel resistance in breast cancer cells. Breast Cancer Res 2004; 6: R601-607.

147 Foster JS et al. Estrogens down-regulate p27Kip1 in breast cancer cells through Skp2 and through nuclear export mediated by the ERK pathway. $J$ Biol Chem 2003; 278: 41355-41366. 
148 Viglietto $\mathrm{G}$ et al. Cytoplasmic relocalization and inhibition of the cyclindependent kinase inhibitor p27(Kip1) by PKB/Akt-mediated phosphorylation in breast cancer. Nat Med 2002; 8: 1136-1144.

149 Shibahara T et al. Down-regulation of Skp2 is correlated with p27associated cell cycle arrest induced by phenylacetate in human prostate cancer cells. Anticancer Res 2005; 25: 1881-1888.

150 Li R et al. Biological correlates of p27 compartmental expression in prostate cancer. J Urol 2006; 175: 528-532.

151 Li P et al. p27(Kip1) stabilization and G(1) arrest by 1,25-dihydroxyvitamin $\mathrm{D}(3)$ in ovarian cancer cells mediated through down-regulation of cyclin E/cyclin-dependent kinase 2 and Skp1-Cullin-F-box protein/Skp2 ubiquitin ligase. J Biol Chem 2004; 279: 25260-25267.

152 Rosen DG et al. Subcellular localization of p27kip1 expression predicts poor prognosis in human ovarian cancer. Clin Cancer Res 2005; 11: 632637.

153 Hershko DD, Shapira M. Prognostic role of p27Kip1 deregulation in colorectal cancer. Cancer 2006; 107: 668-675.

154 Piva $R$ et al. Proteasome-dependent degradation of p27/kip1 in gliomas. $J$ Neuropathol Exp Neurol 1999; 58: 691-696.

155 Chiarle $\mathrm{R}$ et al. Increased proteasome degradation of cyclin-dependent kinase inhibitor p27 is associated with a decreased overall survival in mantle cell lymphoma. Blood 2000; 95: 619-626.

156 Motomura W et al. Growth arrest by troglitazone is mediated by p27Kip1 accumulation, which results from dual inhibition of proteasome activity and Skp2 expression in human hepatocellular carcinoma cells. Int $J$ Cancer 2004; 108: 41-46.

157 Nan KJ, Jing Z, Gong L. Expression and altered subcellular localization of the cyclin-dependent kinase inhibitor p27Kip1 in hepatocellular carcinoma. World J Gastroenterol 2004; 10: 1425-1430.

158 Tompa P, Szasz C, Buday L. Structural disorder throws new light on moonlighting. Trends Biochem Sci 2005; 30: 484-489.

159 Bourhis JM et al. The intrinsically disordered C-terminal domain of the measles virus nucleoprotein interacts with the $\mathrm{C}$-terminal domain of the phosphoprotein via two distinct sites and remains predominantly unfolded. Protein Sci 2005; 14: 1975-1992.

160 Fink AL. Natively unfolded proteins. Curr Opin Struct Biol 2005; 15: 35-41. 
161 Uversky VN, Gillespie JR, Fink AL. Why are "natively unfolded" proteins unstructured under physiologic conditions? Proteins 2000; 41: 415-427.

162 Wright PE, Dyson HJ. Intrinsically unstructured proteins: re-assessing the protein structure-function paradigm. J Mol Biol 1999; 293: 321-331.

163 Uversky VN. What does it mean to be natively unfolded? Eur J Biochem 2002; 269: 2-12.

164 Dunker AK et al. Intrinsically disordered protein. J Mol Graph Model 2001; 19: 26-59.

165 Tompa $P$. The interplay between structure and function in intrinsically unstructured proteins. FEBS Lett 2005; 579: 3346-3354. Epub 2005 Apr 3348.

166 Dunker AK et al. Intrinsic protein disorder in complete genomes. Genome Inform Ser Workshop Genome Inform 2000; 11: 161-171.

167 Weiss MA et al. Folding transition in the DNA-binding domain of GCN4 on specific binding to DNA. Nature 1990; 347: 575-578.

168 Bracken C, Carr PA, Cavanagh J, Palmer AG, 3rd. Temperature dependence of intramolecular dynamics of the basic leucine zipper of GCN4: implications for the entropy of association with DNA. J Mol Biol 1999; 285: 2133-2146.

169 O'Neil KT, Hoess RH, DeGrado WF. Design of DNA-binding peptides based on the leucine zipper motif. Science 1990; 249: 774-778.

170 Zhou P et al. Solution structure of the core NFATC1/DNA complex. Cell 1998; 92: 687-696.

171 Radhakrishnan I et al. Solution structure of the KIX domain of CBP bound to the transactivation domain of CREB: a model for activator:coactivator interactions. Cell 1997; 91: 741-752.

172 Radhakrishnan I, Perez-Alvarado GC, Dyson HJ, Wright PE. Conformational preferences in the Ser133-phosphorylated and nonphosphorylated forms of the kinase inducible transactivation domain of CREB. FEBS Lett 1998; 430: 317-322.

173 Dawson $\mathrm{R}$ et al. The N-terminal domain of p53 is natively unfolded. $\mathrm{J} \mathrm{Mol}$ Biol 2003; 332: 1131-1141.

174 Kussie PH et al. Structure of the MDM2 oncoprotein bound to the p53 tumor suppressor transactivation domain. Science 1996; 274: 948-953. 
175 Uesugi $M$ et al. Induced alpha helix in the VP16 activation domain upon binding to a human TAF. Science 1997; 277: 1310-1313.

176 Tan R, Frankel AD. Costabilization of peptide and RNA structure in an HIV Rev peptide-RRE complex. Biochemistry 1994; 33: 14579-14585.

177 Battiste JL et al. Alpha helix-RNA major groove recognition in an HIV-1 rev peptide-RRE RNA complex. Science 1996; 273: 1547-1551.

178 Puglisi JD, Chen L, Blanchard S, Frankel AD. Solution structure of a bovine immunodeficiency virus Tat-TAR peptide-RNA complex. Science 1995; 270: 1200-1203.

179 Ye X, Kumar RA, Patel DJ. Molecular recognition in the bovine immunodeficiency virus Tat peptide-TAR RNA complex. Chem Biol 1995; 2: 827-840.

180 Hershey PE et al. The Cap-binding protein elF4E promotes folding of a functional domain of yeast translation initiation factor elF4G1. J Biol Chem 1999; 274: 21297-21304.

181 Permyakov SE et al. Natively unfolded C-terminal domain of caldesmon remains substantially unstructured after the effective binding to calmodulin. Proteins 2003; 53: 855-862.

182 Irobi $\mathrm{E}$ et al. Structural basis of actin sequestration by thymosin-beta4: implications for WH2 proteins. The EMBO J 2004; 23: 3599-3608.

183 Gigant B et al. The 4 A X-ray structure of a tubulin:stathmin-like domain complex. Cell 2000; 102: 809-816.

184 Tompa P. Intrinsically unstructured proteins. Trends Biochem Sci 2002; 27: 527-533.

185 Bracken C, lakoucheva LM, Romero PR, Dunker AK. Combining prediction, computation and experiment for the characterization of protein disorder. Curr Opin Struct Biol 2004; 14: 570-576.

186 Ward JJ et al. Prediction and functional analysis of native disorder in proteins from the three kingdoms of life. J Mol Biol 2004; 337: 635-645.

187 Linding R, Russell RB, Neduva V, Gibson TJ. GlobPlot: Exploring protein sequences for globularity and disorder. Nucleic Acids Res 2003; 31: 37013708.

188 Kriwacki RW et al. Structural studies of p21(waf1/cip1/sdi1) in the free and Cdk2-bound state: Conformational disorder mediates binding diversity. Proc Natl Acad Sci USA 1996; 93: 11504-11509. 
189 Hu T, Krezel AM, Li C, Coffey RJ. Structural studies of human Naked2: a biologically active intrinsically unstructured protein. Biochem Biophys Res Commun 2006; 350: 911-915.

190 Hinds MG et al. Bim, Bad and Bmf: intrinsically unstructured BH3-only proteins that undergo a localized conformational change upon binding to prosurvival Bcl-2 targets. Cell Death Differ 2007; 14: 128-136.

191 Nardini M et al. The C-terminal domain of the transcriptional corepressor CtBP is intrinsically unstructured. Protein Sci 2006; 15: 1042-1050.

192 Bourhis JM et al. The C-terminal domain of measles virus nucleoprotein belongs to the class of intrinsically disordered proteins that fold upon binding to their physiological partner. Virus Res 2004; 99: 157-167.

193 Domanski $\mathrm{M}$ et al. Coupling of folding and binding of thymosin beta4 upon interaction with monomeric actin monitored by nuclear magnetic resonance. J Biol Chem 2004; 279: 23637-23645.

194 Schmitz ML et al. Structural and functional analysis of the NF-kappa B p65 $\mathrm{C}$ terminus. An acidic and modular transactivation domain with the potential to adopt an alpha-helical conformation. J Biol Chem 1994; 269: 25613-25620.

195 De Guzman RN, Martinez-Yamout MA, Dyson HJ, Wright PE. Interaction of the TAZ1 domain of the CREB-binding protein with the activation domain of CITED2: regulation by competition between intrinsically unstructured ligands for non-identical binding sites. J Biol Chem 2004; 279: 3042-3049.

196 Love JJ et al. The LEF-1 high-mobility group domain undergoes a disorder-to-order transition upon formation of a complex with cognate DNA. Biochemistry 2004; 43: 8725-8734.

197 Lacy ER et al. p27 binds cyclin-CDK complexes through a sequential mechanism involving binding-induced protein folding. Nat Struct Mol Biol 2004; 11: 358-364.

198 Adkins JN, Lumb KJ. Intrinsic structural disorder and sequence features of the cell cycle inhibitor p57Kip2. Proteins 2002; 46: 1-7.

199 Sivakolundu SG, Bashford D, Kriwacki RW. Disordered p27(Kip1) exhibits intrinsic structure resembling the cdk2/Cyclin A-bound conformation. $J \mathrm{Mol}$ Biol 2005; 353: 1118-1128. Epub 2005 Sep 1120.

200 Wright PE, Dyson HJ. Intrinsically unstructured proteins: re-assessing the protein structure-function paradigm. J Mol Biol 1999; 293: 321-331. 
201 Lacy ER et al. Molecular basis for the specificity of p27 toward cyclindependent kinases that regulate cell division. J Mol Biol 2005; 349: 764773. Epub 2005 Apr 2026.

202 Bienkiewicz EA, Adkins JN, Lumb KJ. Functional consequences of preorganized helical structure in the intrinsically disordered cell-cycle inhibitor p27(Kip1). Biochemistry 2002; 41: 752-759.

203 Harper JW et al. Inhibition of cyclin-dependent kinases by p21. Mol Biol Cell 1995; 6: 387-400.

204 Spolar RS, Record MT, Jr. Coupling of local folding to site-specific binding of proteins to DNA. Science 1994; 263: 777-784.

205 Spolar RS, Livingstone JR, Record MT. Use of liquid hydrocarbon and amide transfer data to estimate contributions to thermodynamic functions of protein folding from the removal of nonpolar and polar surface from water. Biochemistry 1992; 31: 3947-3955.

206 GETAREA. http://www.scsb.utmb.edu/cgi-bin/get a form.tcl, accessed October 6. 2003.

207 Chakrabartty A, Kortemme T, Baldwin RL. Helix propensities of the amino acids measured in alanine-based peptides without helix-stabilizing sidechain interactions. Protein Sci 1994; 3: 843-852.

208 Pace CN, Scholtz JM. A helix propensity scale based on experimental studies of peptides and proteins. Biophys J 1998; 75: 422-427.

209 Chou PY, Fasman GD. Conformational parameters for amino acids in helical, (-sheet, and random coil regions calculated from proteins. Biochemistry 1974; 13: 211-221.

210 Lopez-Llano J, Campos LA, Sancho J. Alpha-helix stabilization by alanine relative to glycine: roles of polar and apolar solvent exposures and of backbone entropy. Proteins 2006; 64: 769-778.

211 Luo P, Baldwin RL. Origin of the different strengths of the $(i, i+4)$ and $(i, i+3)$ leucine pair interactions in helices. Biophys Chem 2002; 96: 103-108.

212 Luo P, Baldwin RL. Origin of the different strengths of the $(i, i+4)$ and $(i, i+3)$ leucine pair interactions in helices. Biophys Chem 2002; 96: 103-108.

213 Klingler TM, Brutlag DL. Discovering structural correlations in alphahelices. Protein Sci 1994; 3: 1847-1857.

214 Moser R. Design, synthesis and structure of an amphipathic peptide with pH-inducible haemolytic activity. Protein Eng 1992; 5: 323-331. 
215 Shoemaker KR et al. Nature of the charged-group effect on the stability of the C-peptide helix. Proc Natl Acad Sci U S A 1985; 82: 2349-2353.

216 Bowman P, Galea CA, Lacy E, Kriwacki RW. Thermodynamic characterization of interactions between p27(Kip1) and activated and nonactivated Cdk2: intrinsically unstructured proteins as thermodynamic tethers. Biochim Biophys Acta 2006; 1764: 182-189. Epub 2006 Jan 2011.

217 Thuret JY, Valay JG, Faye G, Mann C. Civ1 (CAK in vivo), a novel Cdkactivating kinase. Cell 1996; 86: 565-576.

218 Morrow CD, Park J, Wakefield JK. Viral gene products and replication of the human immunodeficiency type 1 virus. Am J Physiol 1994; 266: C1135-1156.

219 Coffin JM. Genes responsible for transformation by avian RNA tumor viruses. Cancer Res 1976; 36: 4282-4288.

220 Collins JK, Chesebro B. Replication-defective Friend murine leukemia virus particles containing uncleaved gag polyproteins and decreased levels of envelope glycoprotein. J Virol 1981; 37: 161-170.

221 Joshi S, Van Brunschot A, Robson I, Bernstein A. Efficient replication, integration, and packaging of retroviral vectors with modified long terminal repeats containing the packaging signal. Nucleic Acids Res 1990; 18: 4223-4226.

222 Markowitz D, Goff S, Bank A. A safe packaging line for gene transfer: separating viral genes on two different plasmids. J Virol 1988; 62: 11201124.

223 Stuhlmann H, Cone R, Mulligan RC, Jaenisch R. Introduction of a selectable gene into different animal tissue by a retrovirus recombinant vector. Proc Natl Acad Sci U S A 1984; 81: 7151-7155.

224 Cone RD, Mulligan RC. High-efficiency gene transfer into mammalian cells: generation of helper-free recombinant retrovirus with broad mammalian host range. 1984. Biotechnology 1992; 24: 420-424.

225 Miller AD. Retrovirus packaging cells. Hum Gene Ther 1990; 1: 5-14.

226 Miller AD, Buttimore $C$. Redesign of retrovirus packaging cell lines to avoid recombination leading to helper virus production. Mol Cell Biol 1986; 6: 2895-2902.

227 Danos O, Mulligan RC. Safe and efficient generation of recombinant retroviruses with amphotropic and ecotropic host ranges. Proc Natl Acad Sci U S A 1988; 85: 6460-6464. 
228 Gill SC, von Hippel PH. Calculation of protein extinction coefficients from amino acid sequence data. Anal Biochem 1989; 182: 319-326.

229 Gasteiger $\mathrm{E}$ et al. Protein identification and analysis tools on the ExPASy server. In: Walker JM (ed). Proteomics Protocols Handbook. Humana Press: Totowa, NJ, 2005.

230 Wiseman T, Williston S, Brandts JF, Lin LN. Rapid measurement of binding constants and heats of binding using a new titration calorimeter. Anal Biochem 1989; 179: 131-137.

231 Pace CN, Scholtz JM. Measuring the conformational stability of a protein. In: Creighton TE (ed). Protein Structure: A Practical Approach. IRL Press: New York, NY, 1989, pp. 299-321.

232 Jainchill JL, Aaronson SA, Todaro GJ. Murine sarcoma and leukemia viruses: assay using clonal lines of contact-inhibited mouse cells. J Virol 1969; 4: 549-553.

233 Brady GP, Sharp KA. Entropy in protein folding and in protein--protein interactions. Current Opinion in Structural Biology 1997; 7: 215.

234 Forman-Kay JD. The 'dynamics' in the thermodynamics of binding. Nat Struct Mol Biol 1999; 6: 1086. 


\section{Appendixes}

\section{A.1 Buffers}

Pre-scission cleavage buffer $(\mathrm{P})$

$50 \mathrm{mM}$ Tris $\mathrm{pH} 7.0$

$150 \mathrm{mM} \mathrm{NaCl}$

1 mM EDTA

$1 \mathrm{mM}$ DTT

Gel filtration buffer (GF)

$20 \mathrm{mM}$ HEPES pH 7.5

$300 \mathrm{mM} \mathrm{NaCl}$

5 mM DTT

Nickel binding buffer (Ni-A)

$20 \mathrm{mM}$ Tris $\mathrm{pH} 8.0$

$5 \mathrm{mM}$ imidazole

$500 \mathrm{mM} \mathrm{NaCl}$

Nickel elution buffer (Ni-B)

$20 \mathrm{mM}$ Tris pH 8.0

$500 \mathrm{mM}$ imidazole

$500 \mathrm{mM} \mathrm{NaCl}$

GST binding buffer (GST-A) $\mathrm{pH} 7.3$

$140 \mathrm{mM} \mathrm{NaCl}$

$2.7 \mathrm{mM} \mathrm{KCl}$

$10 \mathrm{mM} \mathrm{Na}_{2} \mathrm{HPO}_{4}$

$1.8 \mathrm{mM} \mathrm{KH}_{2} \mathrm{PO}_{4}$

$5 \mathrm{mM}$ DTT

GST elution buffer (GST-B)

$50 \mathrm{mM}$ Tris $\mathrm{pH} 8.0$

$10 \mathrm{mM}$ reduced glutathione

5 mM DTT 
Q-sepahrose column binding buffer (Q-A)

$20 \mathrm{mM}$ HEPES $\mathrm{pH} 7.5$

$20 \mathrm{mM} \mathrm{NaCl}$

5 mM DTT

Q-sepahrose column elution buffer (Q-B)

20 mM HEPES pH 7.5

$1.0 \mathrm{M} \mathrm{NaCl}$

$5 \mathrm{mM}$ DTT

Kinase assay buffer (KA)

$20 \mathrm{mM}$ HEPES $\mathrm{pH} 7.3$

$25 \mathrm{mM}$ sodium b-glycerolphosphate

$15 \mathrm{mM} \mathrm{MgCl}_{2}$

$16 \mathrm{mM}$ EGTA

$0.5 \mathrm{mM}$ activated $\mathrm{Na}_{3} \mathrm{VO}_{4}$

$10 \mathrm{mM}$ DTT

HPLC column binding buffer (HPLC-A)

$0.1 \%$ TFA (v/v) in $\mathrm{H}_{2} \mathrm{O}$

HPLC column elution buffer (HPLC-B)

$0.1 \%$ TFA in acetonitrile

Thrombin cleavage buffer for cyclin A (TcycA)

$20 \mathrm{mM}$ Tris $\mathrm{pH} 8.0$

$1.0 \mathrm{M} \mathrm{NaCl}$

$2.5 \mathrm{mM} \mathrm{CaCl}_{2}$

Nickel binding buffer for D2 peptide (Ni-A/D2)

$20 \mathrm{mM} \mathrm{Na}_{2} \mathrm{HPO}_{4} .7 \mathrm{H}_{2} \mathrm{O} \mathrm{pH} 6.5$

$500 \mathrm{mM} \mathrm{NaCl}$

$5 \mathrm{mM}$ imidazole

Nickel elution buffer for D2 peptide (Ni-B/D2)

$20 \mathrm{mM} \mathrm{Na}_{2} \mathrm{HPO}_{4} .7 \mathrm{H}_{2} \mathrm{O} \mathrm{pH} 6.5$

$500 \mathrm{mM} \mathrm{NaCl}$

$500 \mathrm{mM}$ imidazole 
Thrombin cleavage buffer $(T)$

$20 \mathrm{mM}$ Tris pH 8.0

$50 \mathrm{mM} \mathrm{Nacl}$

5 mM DTT

HPLC sample buffer (D)

$10 \mathrm{mM}$ Tris $\mathrm{pH} 7.2$

$5 \mathrm{mM} \mathrm{NaCl}$

CD buffer

$10 \mathrm{mM}$ phosphate $\mathrm{pH} 7.0$

5 mM DTT

Thermal denaturation buffer

$1 \mathrm{mM}$ phosphate $\mathrm{pH} 7.0$

$25 \mathrm{mM} \mathrm{NaCl}$

$1 \mathrm{mM}$ DTT 


\section{A.2 Growth media}

Luria-Bertani media (LB)

Tryptone

$10 \mathrm{~g}$

Yeast extract

$5 \mathrm{~g}$

$\mathrm{NaCl}$

$10 \mathrm{~g}$

Antibiotic (kanamycin $30 \mathrm{mg} / \mathrm{ml}$ stock, ampicillin $50 \mathrm{mg} / \mathrm{ml}$ stock) $\mathrm{H}_{2} \mathrm{O}$ $1 \mathrm{~mL}$ $1 \mathrm{~L}$ 


\section{A.2 Tables and figures}

Table A.1 Sequences of oligonucleotides used in preparing mutagenesis cassettes for the p27-KID mutants.

p27-KID mutant

Oligonucleotide sequences

\begin{tabular}{|c|c|}
\hline \multirow[t]{2}{*}{ p27-KID ${ }^{p 21 \text { helix }}$} & $\begin{array}{c}5^{\prime} \text { GTAACGCATATGGAGCACCCCAAGCCCTCGGCCTGCAGGAACCTCT } \\
\text { TCGGCCCGGTGGACAGCGAACAGCTGAGCCGCGATTGCGATGCGCTGA } \\
\text { TGGCGGGCTG } 3^{\prime}\end{array}$ \\
\hline & $\begin{array}{c}5^{\prime} \text { CCCCGCCTCGAGGGGTTTGTGATTCTGAAAATCGAAATTCCAGCGT } \\
\text { TCGCGCGCTTCCTGAATGCAGCCCGCCATCAGCGCATCGCAATCGCGG } \\
\text { CTCAGCTGTT } 3^{\prime}\end{array}$ \\
\hline \multirow[t]{2}{*}{ p27-KID ${ }^{\text {p57helix }}$} & $\begin{array}{c}5^{\prime} \text { GTAACGCATATGGAGCACCCCAAGCCCTCGGCCTGCAGGAACCTCT } \\
\text { TCGGCCCGGTGGACCATGAAGAACTGAGCCGCGAACTGCAGGCGCGCC } \\
\text { TGGCGGAACT } 3^{\prime}\end{array}$ \\
\hline & $\begin{array}{c}5^{\prime} \text { CCCCGCCTCGAGGGGTTTGTGATTCTGAAAATCGAAATTCCAGCGG } \\
\text { TTCTGATCTTCCGCGTTCAGTTCCGCCAGGCGCGCCTGCAGTTCGCGG } \\
\text { CTCAGTTCTT } 3^{\prime}\end{array}$ \\
\hline \multirow[t]{2}{*}{$\mathrm{p} 27-\mathrm{KID}^{\Delta \mathrm{C}}$} & $\begin{array}{c}\text { 5' GCCTCCCATATGGAGCACCCCAAGCCCTCGGCCTGCAGGAACCTCT } \\
\text { TCGGCCCGGTGGACCACGGCGAGGGCACCGGCGACTTGGAGAAGCACT } \\
\text { GCAGAGACAT } 3^{\prime}\end{array}$ \\
\hline & $\begin{array}{c}5^{\prime} \text { CTTGGGCTCGAGGGGTTTGTGATTCTGAAAATCGAAATTCCACTTG } \\
\text { CGCTGGCTCGCCTCTTCCATGTCTCTGCAGTGCTTCTCCAAGTCGCCG } \\
\text { GTGCCCTCGC } 3^{\prime}\end{array}$ \\
\hline \multirow[t]{2}{*}{$\mathrm{p} 27-\mathrm{KID}^{\mathrm{A} 10}$} & $\begin{array}{c}5^{\prime} \text { GCCTCCCATATGGAGCACCCCAAGCCCTCGGCCTGCAGGAACCTCT } \\
\text { TCGGCCCGGTGGACCACGCGGCGTTAACCGCGGACTTGGAGGCGCACT } \\
\text { GCGCGGCGAT } 3^{\prime}\end{array}$ \\
\hline & $\begin{array}{c}5^{\prime} \text { CTTGGGCTCGAGGGGTTTGTGATTCTGAAAATCGAAATTCCACGCC } \\
\text { GCCTGGCTCGCCGCTTCCATCGCCGCGCAGTGCGCCTCCAAGTCCGCG } \\
\text { GTTAACGCCG } 3^{\prime}\end{array}$ \\
\hline
\end{tabular}

(continued) 
Table A.1 (continued).

p27-KID mutant Oligonucletide sequences

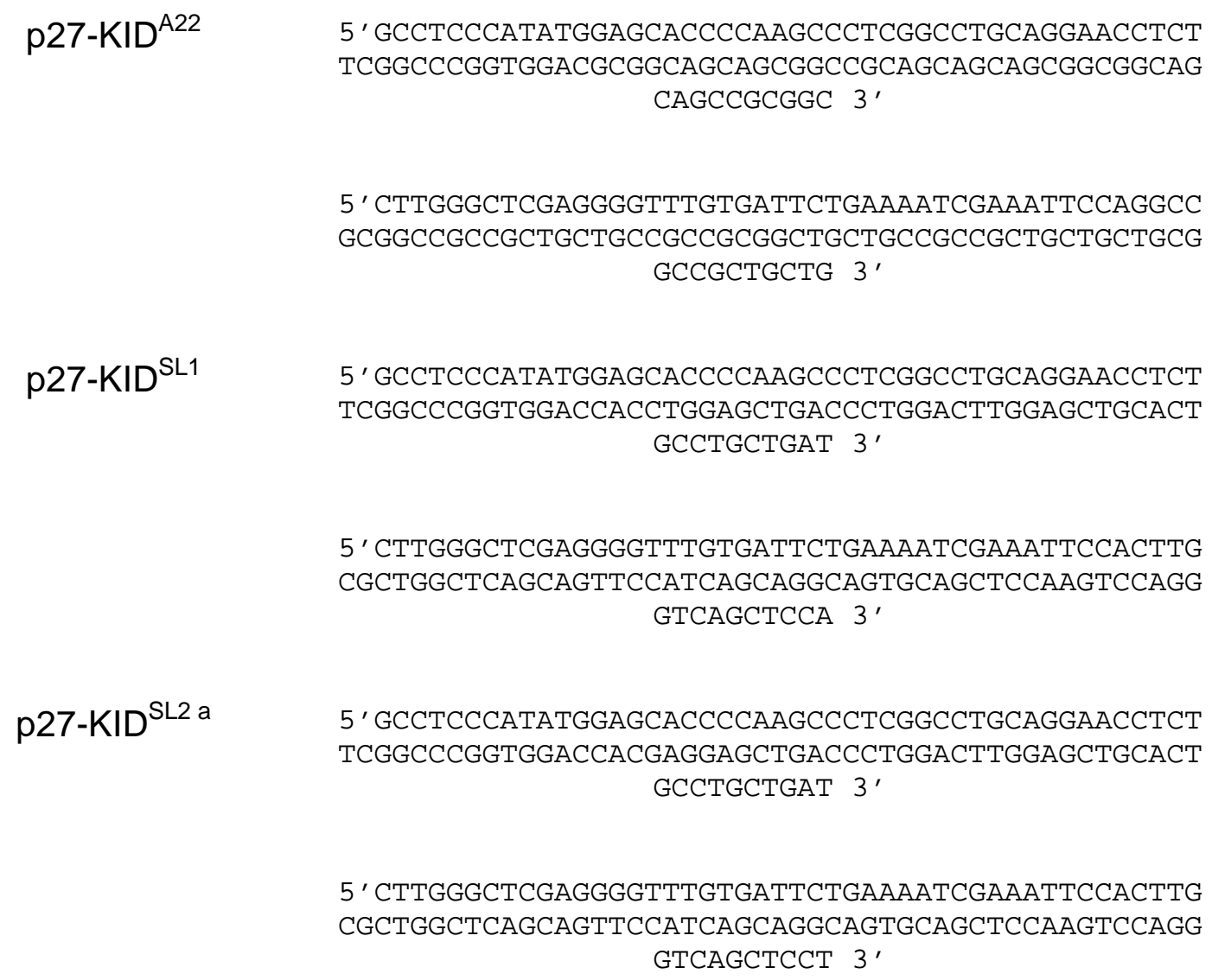

(continued) 


\section{Table A.1 (continued)}

\section{p27-KID mutant Oligonucleotide sequences}

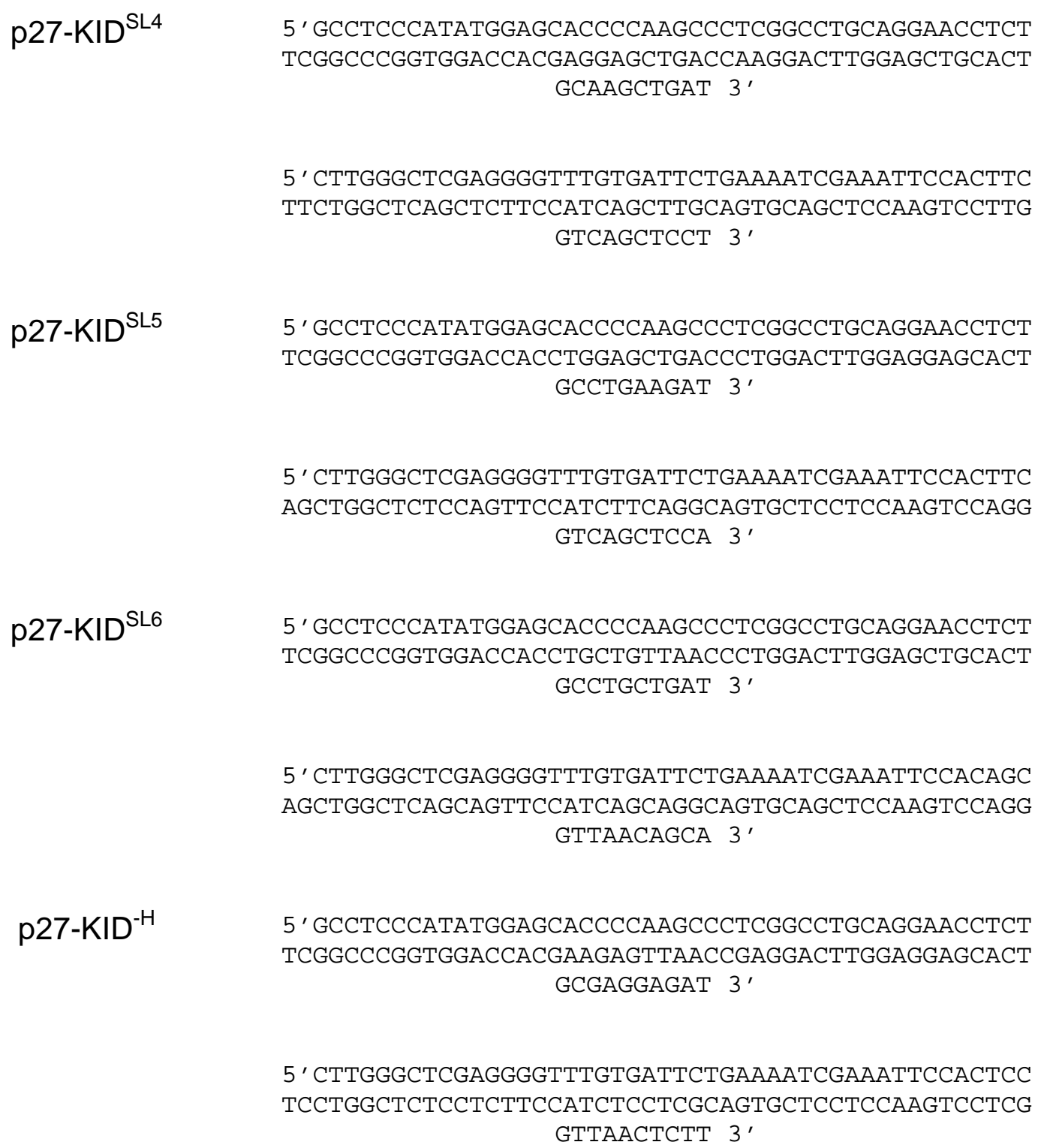

(continued) 
Table A.1 (continued)

p27-KID mutant

Oligonucleotide sequences

\begin{tabular}{|c|c|}
\hline p27-KID ${ }^{\text {loop }}$ & $\begin{array}{c}5^{\prime} \text { GCCTCCCATATGGAGCACCCCAAGCCCTCGGCCTGCAGGAACCTCT } \\
\text { TCGGCCCGGTGGACACCAACCCGGGCAACGGCGGCCATGGCGGCACCG } \\
\text { GCGTGGGCGG } 3^{\prime}\end{array}$ \\
\hline & $\begin{array}{c}5^{\prime} \text { CTTGGGCTCGAGGGGTTTGTGATTCTGAAAATCGAAATTCCAGCCA } \\
\text { TGGCCGTTGCCGCCGTTGCCGCCCACGCCGGTGCCGCCATGGCCGCCG } \\
\text { TTGCCCGGGT } 3^{\prime}\end{array}$ \\
\hline
\end{tabular}


Table A.2 Reagents for overlap extension PCR.

\begin{tabular}{cc}
\hline Reagent & Volume $(\mu \mathrm{L})$ \\
\hline Forward oligonucleotide $(10 \mathrm{ng} / \mu \mathrm{l})$ & 1 \\
Reverse oligonucleotide $(10 \mathrm{ng} / \mu \mathrm{l})$ & 1 \\
Forward end primer $(125 \mathrm{ng} / \mu \mathrm{l})$ & 1 \\
Reverse end primer $(125 \mathrm{ng} / \mu \mathrm{l})$ & 1 \\
Deoxynucleosides (dNTP's), $10 \mathrm{mM}$ & 1 \\
PCR buffer & 5 \\
Enzyme $^{\mathrm{a}}$ & 1 \\
Distilled, deionized water & 40 \\
Total Reaction Volume & 50 \\
\hline
\end{tabular}

${ }^{a}$ The enzyme used was the expand high fidelity PCR system by Roche (Mannheim, Germany). 
Table A.3 Thermocycler settings for the oePCR.

\begin{tabular}{ccc}
\hline Step & Temperature & Time \\
\hline 1 & $95^{\circ} \mathrm{C}$ & 45 seconds \\
2 & Start cycle & \\
3 & $95^{\circ} \mathrm{C}$ & 45 seconds \\
4 & $55^{\circ} \mathrm{C}$ & 1 minute \\
& $72^{\circ} \mathrm{C}$ & 1 minute \\
5 & Go to step 2 for 20 cycles & \\
6 & $72{ }^{\circ} \mathrm{C}$ & 10 minutes \\
\hline
\end{tabular}


Table A.4 Sequences of oligonucleotides and primers used in preparing cloning cassettes for the Cip/Kip linker domain peptides.

\begin{tabular}{|c|c|}
\hline Peptide & Sequences \\
\hline \multirow[t]{3}{*}{ p27 linker domain } & $\begin{array}{c}\text { 5' AAAAAACCGCGGGGTTCTTCTCATCATCATCATCATCAT } \\
\text { TCTTCTGGTCTGGTTCCACGTGGTTCTCATATGCATGAAGA } \\
\text { ACTGACTCGTGATCTGGA } 3^{\prime}\end{array}$ \\
\hline & $\begin{array}{c}5^{\prime} \text { TTTTTTGAATTCTTATTACCATTTACGTTGAGAAGCTTC } \\
\text { TTCCATATCACGACAATGTTTTCCAGATCACGAGTCAGTT } \\
\text { CTTCATGCATATGAGAAC } 3^{\prime}\end{array}$ \\
\hline & $\begin{array}{l}\text { 5'AAAAAACCGCGGGGTTCTTCTCATCATC 3' } \\
\text { 5' TTTTTTGAATTCTTATTACCATTTACGT } \text { 3' }^{\prime 2}\end{array}$ \\
\hline \multirow[t]{4}{*}{ p21 linker domain } & $\begin{array}{c}\text { 5' AAAAAACCGCGGGGTTCTTCTCATCATCATCATCATCAT } \\
\text { TCTTCTGGTCTGGTTCCACGTGGTTCTCATATGAGCGAACA } \\
\text { GCTGAGCCGCGATTGCGA } 3^{\prime}\end{array}$ \\
\hline & $\begin{array}{c}5^{\prime} \text { TTTTTTGAATTCTTATTACCAGCGTTCGCGCGCTTCCTG } \\
\text { AATGCAGCCCGCCATCAGCGCATCGCAATCGCGGCTCAGCT } \\
\text { GTTCGCTCATATGAGAAC } 3^{\prime}\end{array}$ \\
\hline & 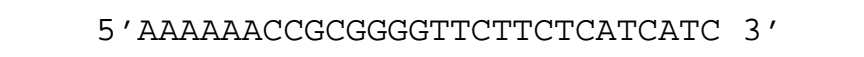 \\
\hline & 5'TTTTTTGAATtCTTATTACCAGCGTTCG $\quad 3{ }^{\prime}$ \\
\hline \multirow[t]{4}{*}{ p57 linker domain } & $\begin{array}{c}5^{\prime} \text { AAAAAACCGCGGGGTTCTTCTCATCATCATCATCATCAT } \\
\text { TCTTCTGGTCTGGTTCCACGTGGTTCTCATATGCATGAAGA } \\
\text { ACTGAGCCGCGACTGCA } 3^{\prime}\end{array}$ \\
\hline & $\begin{array}{c}5^{\prime} \text { TTTTTTGAATTCTTATTACCAGCGGTTCTGATCTTCCGC } \\
\text { GTTCAGTTCCGCCAGGCGCGCCTGCAGTTCGCGGCTCAGTI } \\
\text { CTTCATGCATATGAGAAC } 3^{\prime}\end{array}$ \\
\hline & 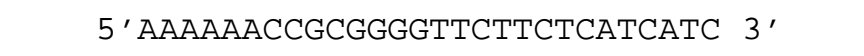 \\
\hline & 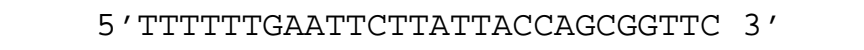 \\
\hline
\end{tabular}


Table A.5 Cloning of the p27-KID linker domain mutants.

\begin{tabular}{|c|c|}
\hline Variant & Vector(s) \\
\hline $\mathrm{p} 27-\mathrm{KID}^{\mathrm{wt}}$ & pET28, pET28GSTHis \\
\hline $\mathrm{p} 27-\mathrm{KID}^{\mathrm{A} 10}$ & pET28, pET28GSTHis \\
\hline $\mathrm{p} 27-\mathrm{KID}^{\mathrm{A} 22}$ & pET28GSTHis \\
\hline $\mathrm{p} 27-\mathrm{KID}^{\mathrm{SL1}}$ & pET28 \\
\hline \multicolumn{2}{|l|}{$\mathrm{p} 27-\mathrm{KID}^{\mathrm{SL2} \text { a }}$} \\
\hline p27-KID ${ }^{S\llcorner 3}\left(p 27-K D^{+H}\right)$ & pET28 \\
\hline $\mathrm{p} 27-\mathrm{KID}^{\mathrm{SL4}}$ & pET28 \\
\hline $\mathrm{p} 27-\mathrm{KID}^{\mathrm{SL5}}$ & pET28 \\
\hline $\mathrm{p} 27-\mathrm{KID}^{\mathrm{SL6}}$ & pET28, pET28GSTHis \\
\hline p27-KID ${ }^{-H}$ & pET28GSTHis \\
\hline p27-KID ${ }^{100 p}$ & pET28GSTHis \\
\hline p27-KID ${ }^{\mathrm{p} 21 \text { helix }}$ & pET28GSTHis \\
\hline p27-KID ${ }^{\text {p57helix }}$ & pET28, pET28GSTHis \\
\hline $\mathrm{p} 27-\mathrm{KID}^{\Delta \mathrm{C}}$ & pET28, pET28GSTHis \\
\hline
\end{tabular}

${ }^{a}$ The cassette for the p27-KID ${ }^{\mathrm{SL2}}$ mutant could not be successfully cloned into either the pET28 or the pET28GSTHis vector. 
Table A.6 Expression and solubility of the p27-KID linker domain mutants.

\begin{tabular}{ccc}
\hline Variant & Expression vector(s) & Solubility \\
\hline $\mathrm{p} 27-\mathrm{KID}^{\mathrm{wt}}$ & $\mathrm{pET28,} \mathrm{pET28GSTHis}$ & Soluble \\
$\mathrm{p} 27-\mathrm{KID}^{\mathrm{A} 10}$ & $\mathrm{pET28GSTHis}$ & Soluble \\
$\mathrm{p} 27-\mathrm{KID}^{\mathrm{A} 22}$ & $\mathrm{pET28GSTHis}$ & Insoluble \\
$\mathrm{p} 27-\mathrm{KID}^{\mathrm{SL1}}$ & $\mathrm{pET28}$ & Insoluble \\
$\mathrm{p} 27-\mathrm{KID}^{\mathrm{SL3}}\left(\mathrm{p} 27-\mathrm{KID}^{+H}\right)$ & $\mathrm{pET28}$ & Partly soluble \\
$\mathrm{p} 27-\mathrm{KID}^{\mathrm{SL4}}$ & $\mathrm{pET28}$ & Soluble \\
$\mathrm{p} 27-\mathrm{KID}^{\mathrm{SL5}}$ & $\mathrm{pET28}$ & Insoluble \\
$\mathrm{p} 27-\mathrm{KID}^{\mathrm{SL6}}$ & $\mathrm{pET28GSTHis}$ & Insoluble \\
$\mathrm{p} 27-\mathrm{KID}^{-H}$ & $\mathrm{pET28GSTHis}$ & Soluble \\
$\mathrm{p} 27-\mathrm{KID}^{\text {loop }}$ & $\mathrm{pET28GSTHis}$ & Soluble \\
$\mathrm{p} 27-\mathrm{KID}^{\mathrm{p} 21 h e l i x}$ & $\mathrm{pET28GSTHis}$ & Soluble \\
$\mathrm{p} 27-\mathrm{KID}^{\mathrm{p} 57 h e l i x}$ & $\mathrm{pET28GSTHis}$ & Soluble \\
$\mathrm{p} 27-\mathrm{KID}^{\Delta C}$ & $\mathrm{pET28GSTHis}$ & Soluble \\
\hline
\end{tabular}




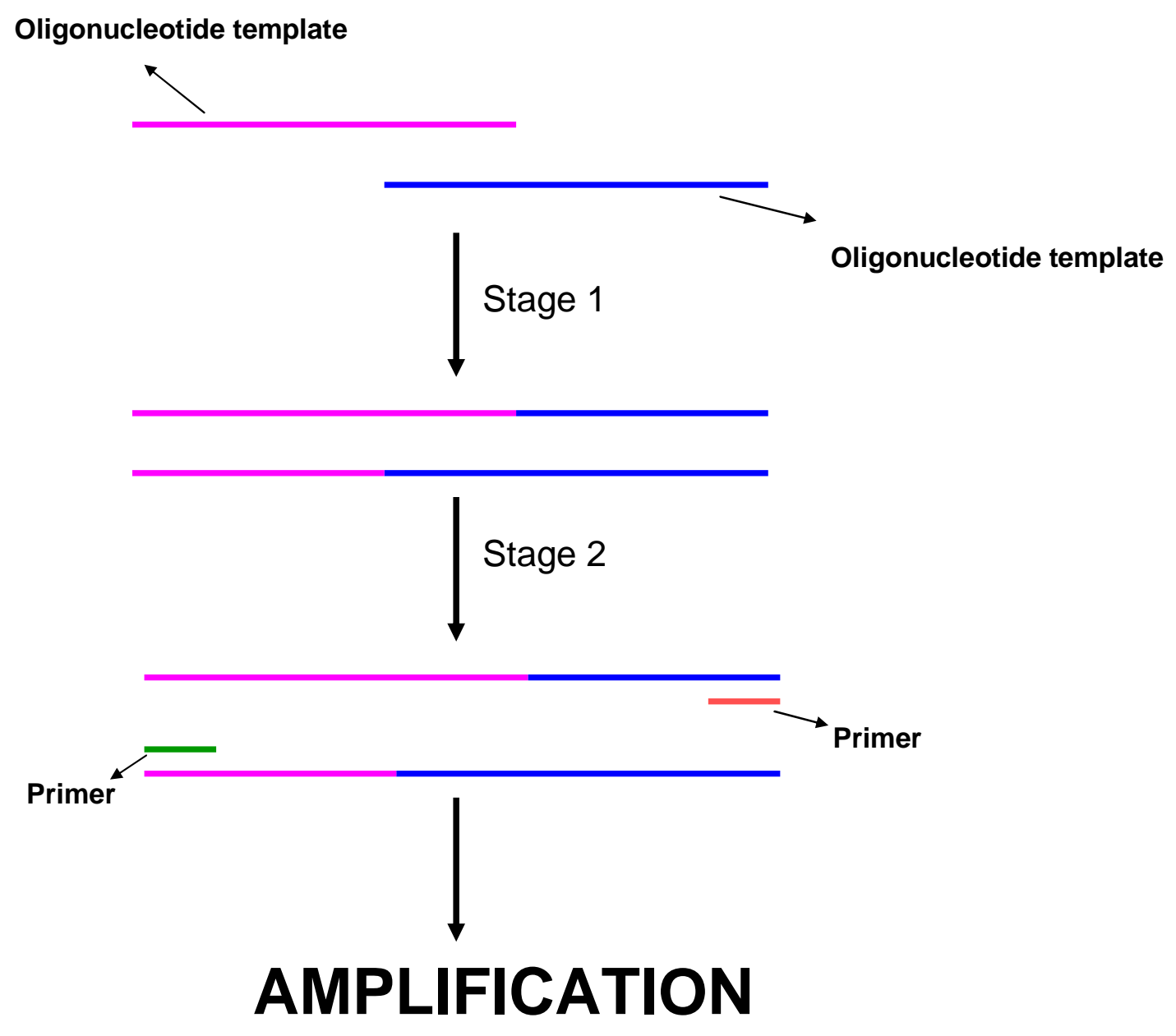

Figure A.1 A schematic representation of oePCR. 


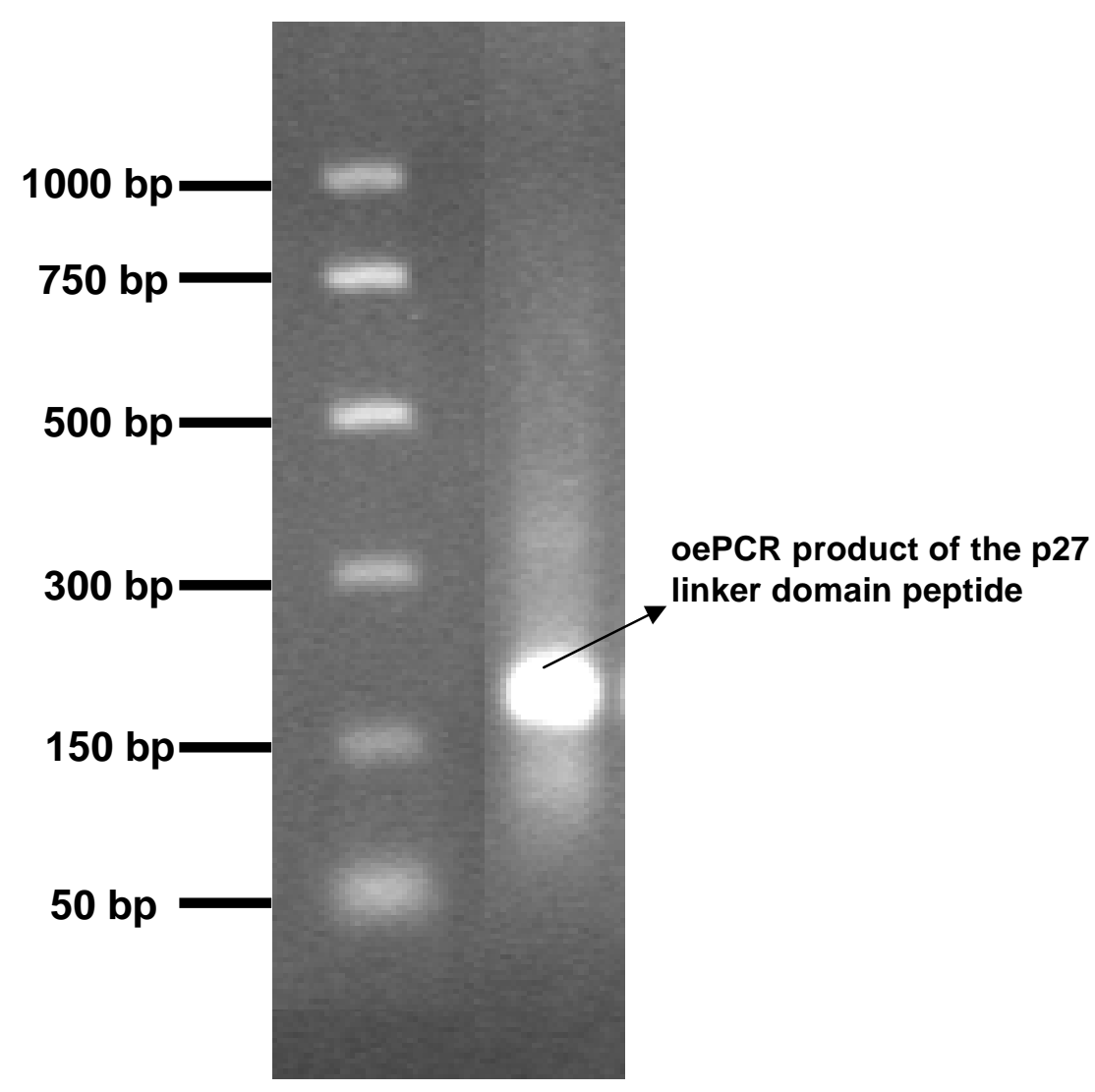

Figure A.2 Agarose gel analysis of the oePCR for the p27 linker domain peptide. The expected product size $159 \mathrm{bp}$. 


\begin{abstract}
TGTGAGCGGATACATTCCCCTCTANAAATCCNCTTTGTTTAAATTTAAGAAGGAGATATAC CATGGGCAGCAGCCATCATCATCATCATCACAGCAGCGGCCTGGTGCCGCGCGGCAGCCAT ATGGAGCACCCCAAGCCCTCGGCCTGCAGGAACCTCTTCGGCCCGGTGGACCACGAAGAG TTAACCCGGGACTTGGAGAAGCACTGCAGAGACATGGAAGAGGCGAGCCAGCGCAAGTGG AATTTCGATTTTCAGAATCACAAACCCCTAGAGGGCAAGTACGAGTGGCAAGAGGTGGAG AAGGGCAGCTTGCCCGAGTTCTACTACAGACCCCCGCGGCCCCCCAAAGGTGCCTGCAAG GTGCCGGCGCAGGAGTAATAAGGATCCGAATTCGAGCTCCGTCGACAAGCTTGCGGCCGC ACTCGAGCACCACCACCACCACCACTGAGATCCGGCTGCTAACAAAGCCCGAAAGGAAGC TGAGTTGGCTGCTGCCACCGCTGAGCAATAACTAGCATAACCCCTTGGGGCCTCTAAACG GGTCTTGAGGGGTTTTTTGCTGAAAGGAGGAACTATATCCGGATTGGCGAATGGGACGCG CCCTGTAGCGGNGCATTAAGCGCGGCGGCTGTGGTGGTTACGCGCCANCGNGACCGCTAC ACTTGCCAGCGCCCTAGCGACCGCTCCTTTCGCTTTCTTCCCTTNCTTTCTCNCCACGTT CGCCGGCTTTTCCTCNTCAAGCTCTAAATCGGGGGCTNCCCTTTAGGGNTCCGNTTTANT GCTTTACGGCACCTCNACCC
\end{abstract}

Figure A.3 Sequencing results for the pET28p27-KID plasmid. This sequence is only for the p27-KID coding region. 


\begin{abstract}
AGAAGGAGATATACCATGGGCAGCAGCCATCATCATCATCATCACAGCAGCGGCCTGGTG CCGCGCGGCAGCCATATGTCAAACGTGCGAGTGTCTAACGGGAGCCCTAGCCTGGAGCGG ATGGACGCCAGGCAGGCGGAGCACCCCAAGCCCTCGGCCTGCAGGAACCTCTTCGGCCCG GTGGACCACGAAGAGTTAACCCGGGACTTGGAGAAGCACTGCAGAGACATGGAAGAGGCG AGCCAGCGCAAGTGGAATTTCGATTTTCAGAATCACAAACCCCTAGAGGGCAAGTACGAG TGGCAAGAGGTGGAGAAGGGCAGCTTGCCCGAGTTCTATTACAGACCCCCGCGGCCCCCC AAAGGTGCCTGCAAGGTGCCGGCGCAGGAGAGCCAGGATGTCAGCGGGAGCCGCCCGGCG GCGCCTTTAATTGGGGCTCCGGCTAACTCTGAGGACACGCATTTGGTGGACCCAAAGACT GATCCGTCGGACAGCCAGACGGGGTTAGCGGAGCAATGCGCAGGAATAAGGAAGCGACCT GCAACCGACGATTCTTCTACTCAAAACAAAAGAGCCAACAGAACAGAAGAAAATGTTTCA GACGGTTCCCCAAATGCCGGTTCTGTGGAGCAGACGCCCAAGAAGCCTGGCCTCAGAAGA CGTCAAACGTAATAAGGATCCGAATTCGAGCTCCGTCGACAAGCTTGCGGCCGCACTCGA
\end{abstract}

Figure A.4 Sequencing results for the pET28flp27 plasmid. This sequence is only for the flp27 coding region. 


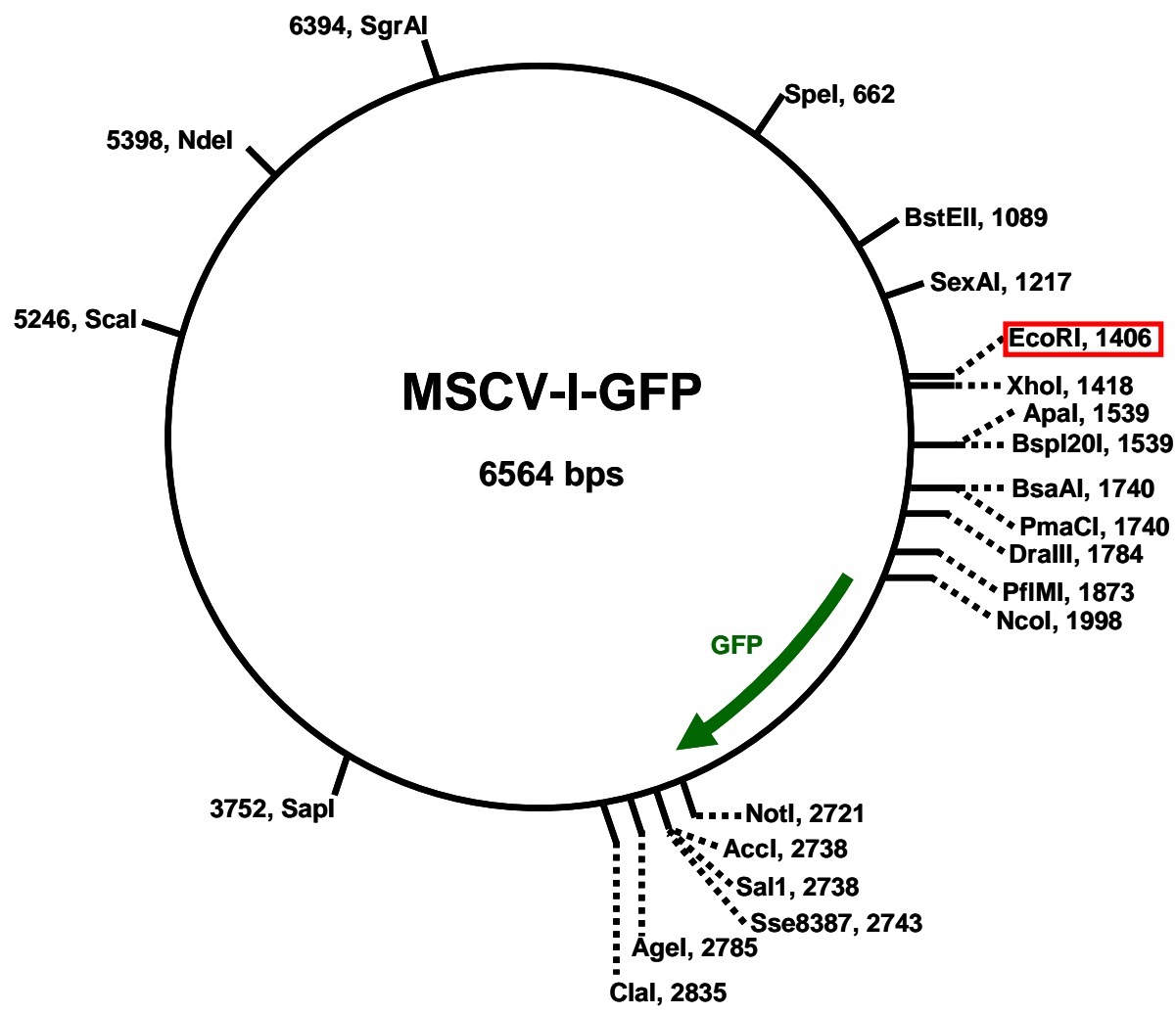

Figure A.5 Vector map for the MSCV-I-GFP plasmid. The highlighted EcoRI site was the cloning site for the flp27 linker domain mutants. 


\section{MSCV-I-GFPflp27 ${ }^{\mathrm{wt}}$}

CTTCTCTAGGCGCCGGAATTCATGTCAAACGTGCGAGTGTCTAACGGGAGCCCTAGCCTGGAGCGGATGGACGCCAGGC AGGCGGAGCACCCCAAGCCCTCGGCCTGCAGGAACCTCTTCGGCCCGGTGGACCACGAAGAGTTAACCCGGGACTTGGA GAAGCACTGCAGAGACATGGAAGAGGCGAGCCAGCGCAAGTGGAATTTCGATTTTCAGAATCACAAACCCCTAGAGGGC AAGTACGAGTGGCAAGAGGTGGAGAAGGGCAGCTTGCCCGAGTTCTATTACAGACCCCCGCGGCCCCCCAAAGGTGCCT GCAAGGTGCCGGCGCAGGAGAGCCAGGATGTCAGCGGGAGCCGCCCGGCGGCGCCTTTAATTGGGGCTCCGGCTAACTC TGAGGACACGCATTTGGTGGACCCAAAGACTGATCCGTCGGACAGCCAGACGGGGTTAGCGGAGCAATGCGCAGGAATA AGGAAGCGACCTGCAACCGACGATTCTTCTACTCAAAACAAAAGAGCCAACAGAACAGAAGAAAATGTTTCAGACGGTT CCCCAAATGCCGGTTCTGTGGAGCAGACGCCCAAGAAGCCTGGCCTCAGAAGACGTCAAACGTATCCGTATGATGTGCC GGATTATGCGTAATAAGAATTCGTTAACCTCGAGCGGGATCAATTCCGCCCCCCCCCTAACGTTACTGGCCGAAGC

\section{MSCV-I-GFPfIp27 $7^{\text {SL3 }}$ (MSCV-I-GFPflp27 $7^{+\mathrm{H})}$}

GCGCCGGANTTCATGTCAAACGTGCGAGTGTCTAACGGGAGCCCTAGCCTGGAGCGGATGGACGCCAGGCAGGCGGAGC ACCCCAAGCCCTCGGCCTGCAGGAACCTCTTCGGCCCGGTGGACCACGAGGAGCTGACCAAGGACTTGGAGCTGCACTG CCTGCTGATGGAACTGCTGAGCCAGCGCAAGTGGAATTTCGATTTTCAGAATCACAAACCCCTCGAGGGCAAGTACGAG TGGCAAGAGGTGGAGAAGGGCAGCTTGCCCGAGTTCTACTACAGACCCCCGCGGCCCCCCAAAGGTGCCTGCAAGGTGC CGGCGCAGGAGAGCCAGGATGTCAGCGGGAGCCGCCCGGCGGCGCCTTTAATTGGGGCTCCGGCTAACTCTGAGGACAC GCATTTGGTGGACCCAAAGACTGATCCGTCGGACAGCCAGACGGGGTTAGCGGAGCAATGCGCAGGAATAAGGAAGCGA CCTGCAACCGACGATTCTTCTACTCAAAACAAAAGAGCCAACAGAACAGAAGAAAATGTTTCAGACGGTTCCCCAAATG CCGGTTCTGTGGAGCAGACGCCCAAGAAGCCTGGCCTCAGAAGACGTCAAACGTATCCGTATGATGTGCCGGATTATGC GTAATAAGAATTCGTTAACCTCGAGCGGGATCAATTCCGCCCCCCCCCTAACGTTACTGGCCGAAGCCGCTTGGAATAA NGCCGGTGTGCGTTTGTCTATATGTTATTTTCCACCATATTG

\section{MSCV-I-GFPfIp27 ${ }^{-H}$}

CCCTTTATCCAGCCCTCACTCCTTCTCTAGGCGCCGGAATTCATGTCAAACGTGCGAGTGTCTAACGGGAGCCCTAGCC TGGAGCGGATGGACGCCAGGCAGGCGGAGCACCCCAAGCCCTCGGCCTGCAGGAACCTCTTCGGCCCGGTGGACCACGA AGAGTTAACCGAGGACTTGGAGGAGCACTGCGAGGAGATGGAAGAGGAGAGCCAGGAGGAGTGGAATTTCGATTTTCAG AATCACAAACCCCTCGAGGGCAAGTACGAGTGGCAAGAGGTGGAGAAGGGCAGCTTGCCCGAGTTCTACTACAGACCCC CGCGGCCCCCCAAAGGTGCCTGCAAGGTGCCGGCGCAGGAGAGCCAGGATGTCAGCGGGAGCCGCCCGGCGGCGCCTTT AATTGGGGCTCCGGCTAACTCTGAGGACACGCATTTGGTGGACCCAAAGACTGATCCGTCGGACAGCCAGACGGGGTTA GCGGAACAATGCGCAGGAATAAGGAAGCGACCTGCAACCGACGATTCTTCTACTCAAAACAAAAGAGCCAACAGAACAG AAGAAAATGTTTCAGACGGTTCCCCGAATGCCGGTTCTGTGGAGCAGACGCCCAAGAAGCCTGGCCTCAGAAGACGTCA AACGTATCCGTATGATGTGCCGGATTATGCGTAATAAGAATTCGTTAACCTCGAGCGGGATCAATTCCGCCCCCCCCCT AACGTTACTGGCCGAAGCC

\section{MSCV-I-GFPflp27 $7^{\text {loop }}$}

GAATTCATGTCAAACGTGNGAGTGTCTAACGGGAGCCCTAGCCTGGAGCGGATGGACGCCAGGCAGGCGGAGCACCCCA AGCCCTCGGCCTGCAGGAACCTCTTCGGCCCGGTGGACACCAACCCGGGCAACGGCGGCCATGGCGGCACCGGCGTGGG CGGCAACGGCGGCAACGGCCATGGCTGGAATTTCGATTTTCAGAATCACAAACCCCTCGAGGGCAAGTACGAGTGGCAA GAGGTGGAGAAGGGCAGCTTGCCCGAGTTCTACTACAGACCCCCGCGGCCCCCCAAAGGTGCCTGCAAGGTGCCGGCGC AGGAGAGCCAGGATGTCAGCGGGAGCCGCCCGGCGGCGCCTTTAATTGGGGCTCCGGCTAACTCTGAGGACACGCATTT GGTGGACCCAAAGACTGATCCGTCGGACAGCCAGACGGGGTTAGCGGAGCAATGCGCAGGAATAAGGAAGCGACCTGCA ACCGACGATTCTTCTACTCAAAACAAAAGAGCCAACAGAACAGAAGAAAATGTTTCAGACGGTTCCCCAAATGCCGGTT CTGTGGAGCAGACGCCCAAGAAGCCTGGCCTCAGAAGACGTCAAACGTATCCGTATGATGTGCCGGATTATGCGTAATA AGAATTCGTTAACCTNGAGCGGGATCAATTCCGCCCCCCCCCTAACGTTACN

Figure A.6 Sequences of the MSCV-I-GFP plasmids. Only the region coding for the flp27 variants is shown. 


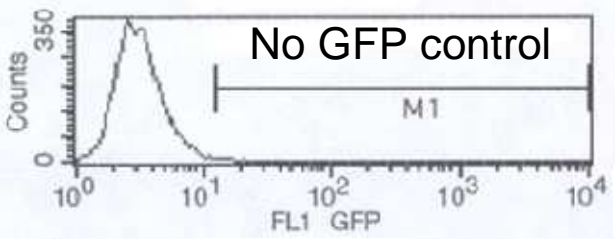

\begin{tabular}{rrrrrr} 
Marker Left, Right & Events & \% Gated $\%$ Total Geo Mean \\
\hline All & 1,9647 & 9133 & 100.00 & 79.13 & 3.13 \\
M1 & 12,9306 & 14 & 0.15 & 0.12 & 1422
\end{tabular}

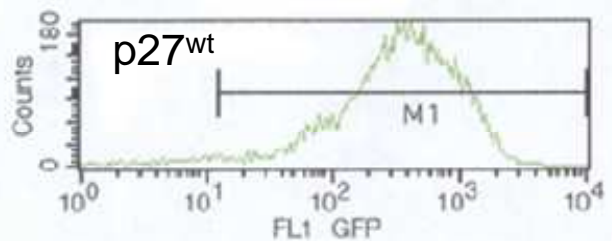

\begin{tabular}{rrrrrr} 
Marker Lett, Right & Events & \% Gated & \% Total Geo Mean \\
\hline All & 1,9647 & 11397 & 100.00 & 65.26 & 297.61 \\
M1 & 12,9306 & 11161 & 97.93 & 63.91 & 323.40
\end{tabular}

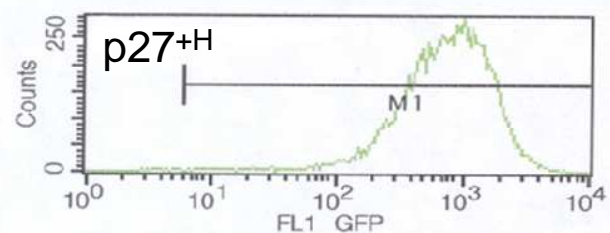

\begin{tabular}{rrrrrr} 
Marker & Left, Fight & Events & $\%$ Gated & $\%$ Total & Geo Mean \\
\hline All & 1,9647 & 13320 & 100.00 & 86.19 & 669.01 \\
M1 & 6,9647 & 13302 & 99.86 & 86.07 & 673.63
\end{tabular}

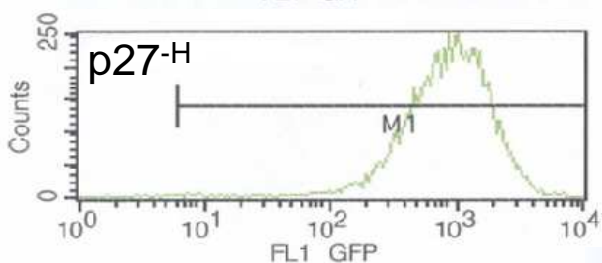

\begin{tabular}{rrrrrr} 
Marker & Left, Right & Events & $\%$ Gated & $\%$ Total & Geo Mean \\
\hline All & 1,9647 & 11021 & 100.00 & 78.36 & 777.47 \\
M1 & 6,9647 & 11007 & 99.87 & 78.26 & 782.68
\end{tabular}

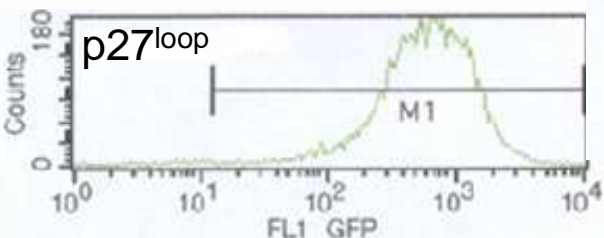

Marker Left. Right Events \% Gated \% Total Geo Mean

\begin{tabular}{rrrrrr}
\hline All & 1,9647 & 10015 & 100.00 & 89.57 & 527.72 \\
M1 & 12,9306 & 9948 & 99.33 & 88.97 & 543.42
\end{tabular}

Figure A.7 Flow cytometry analysis of GFP positive cells. The percentages of GFP positive cells for are circumscribed with red ellipses. 


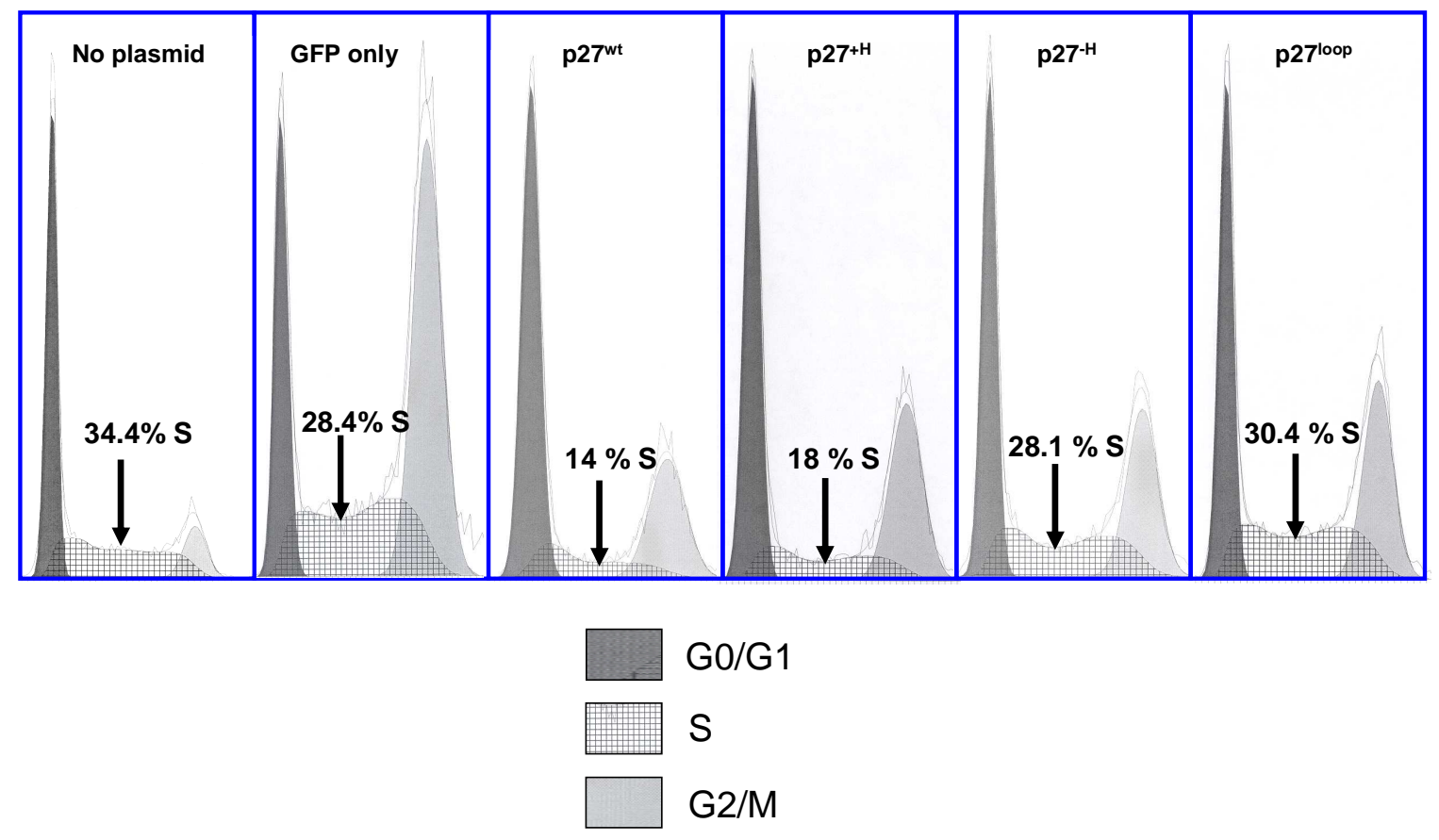

Figure A.8 Cell cycle arrest analysis of the p27-KID mutants. 


\section{Vita}

Steve Biko Otieno was born on March 7, 1979 in Rachuonyo District, Kenya. He attended Maseno National School where he completed his studies in 1996. Steve received a Bachelor of Science degree in Chemistry from Rust College in Holly Springs, MS in 2001 and joined the Molecular Sciences program at The University of Tennessee Health Science Center in the fall of 2001. He began his doctoral work in the laboratory of Dr. Richard Kriwacki at St. Jude Children's Research Hospital in the summer of 2002. Steve expects to graduate with a Ph.D. in Molecular Sciences in May 2007. 